\title{
An Approach To 3D Magnetic Field Calculation Using Numerical and Differential Algebra Methods.
}

S.Caspi, M.Helm L.J. Laslett and V.O. Brady

Lawrence Berkeley Laboratory

University Of California

Berkeley, CA 94720

July 17, 1992

This was supported by the Director, Office of Energy Research, Office of High Energy and Nuclear Physics, High Energy Physics Division, U. S. Department of Energy, under Contract No. DE-AC03-76SF00098. 


\section{Contents}

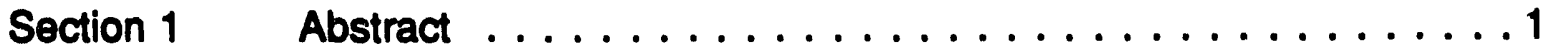

Topic 1 Introduction $\ldots \ldots \ldots \ldots \ldots \ldots \ldots \ldots$

Section 2 Part 1-3D Field Representation. . . . . . . . . . . . 4

Topic 1 Integral Form. . . . . . . . . . . . . . . . 6

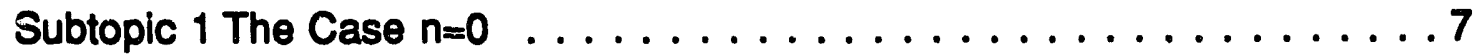

Section 3 The "Biot \& Savart" Field Evaluation . . . . . . . . . . . . . 9

Topic 1 A Numerical Finite-Difference Method . . . . . . . . . . . 10

Topic 2 Differential Algebra Method . . . . . . . . . . . . 13

Subtopic 1 case A . . . . . . . . . . . . . . . 13

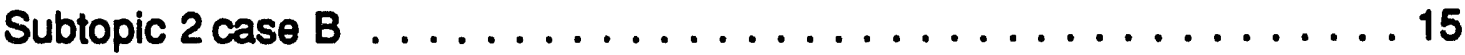

Section 4 Connection to a Fourier-Bessel Series . . . . . . . . . . 16

Section $5 \quad$ Single Particle Orbits . . . . . . . . . . . . . . 21

Section 6 Part 2- Magnetic field in the SSC Quad end. . . . . . . . 25

Section $7 \quad$ Dipole D19 . . . . . . . . . . . . . . . . . 35

Section 8 Part 3-A(z) functions for Dipsle D19, and SSC Quad QC. . . . 37

Appendix A Biot-Savart and DA ................64 


\section{Abstract}

Motivated by the need for new means for specification and determination of 3D fields that are produced by electromagnetic lens elements in the region interior to coil windings and seeking to obtain techniques that will be convenient for accurate conductor placement and dynamical study of particle motion, we have conveniently generalized the representation of a 2D magnetic field to 3D. We have shown that the 3 dimensional magnetic field components of a multipole magnet in the curl-free divergence-free region near the axis $r=0$ can be derived from one dimensional functions $A_{n}(z)$ and their derivatives (part 1). ${ }^{b}$.

In the region interior to coil windings of accelerator magnets the three spatial components of magnet fields can be expressed in terms of "harmonic components" proportional to functions $\sin (n \theta)$ or $\cos (n \theta)$ of the azimuthal angle. The $r, z$ dependence of any such component can then be expressed in terms of powers of $r$ times functions $A_{n}(z)$ and their derivatives. For twodimensional configurations $B_{z}$ of course is identically zero, the derivatives of $A_{n}(z)$ vanish, and the harmonic components of the transverse field then acquire a simple proportionality $B_{r, n} \propto r^{n-1} \sin (n \theta), B_{\theta, n} \propto r^{n-1} \cos (n \theta)$ [for non-skew configurations], whereas in a 3-D configuration the more complex nature of the field gives rise to additional so-called "psuedomultipole" components as judged by additional powers of $\mathrm{r}$ required in the development of the field. Computation of the 3-D magnetic field arising at a sequence of field points, as a direct result of a specified current configuration or coil geometry, can be calculated explicitly through use of the Biot-Savart law and from such data the coefficients can then be derived for a general development of the type indicated above. We indicate, discuss, and illustrate two means by which this development may be performed, employing in the second of these methods computations based on the use of differential algebra with field-point variables representing quantities with respect to which derivatives are evaluated by the computer program.

\section{Introduction}

The performance quality of beam-transport lines and of particle accelerators is basically dependent upon the characteristics of the magnetic fields that normally are employed to guide and focus the beam, the beam behavior being critically influenced by such resonances and nonlinearities as may develop in the course of the particle motion. Quite aside from the complications of collective motion that will develop in the presence of space-charge fields, an analysis of beam dynamics accordingly requires an adequate and convenient specification of the applied fields produced within the beam channel by the current windings of the electromagnetic components of the machine.

The character of such fields of course will be affected by the configuration of any nearby magnetic material as may be present, but evaluation of the supplemental contributions from such magnetizable material will not be discussed here. ${ }^{c}$ The derivation and the description of the three-dimensional (3-D) field produced "directly" by the current windings of magnetic elements

\footnotetext{
b 3D Field Harmonics - S.Caspi , M.Helm , and L.J. Laslett , SC-MAG-328 , LBL-30313, March 1991.

c See, however, a separate report presented at the 1991 Conference on the computation of Electromagnetic Fields (Sorrento, Italy), for inclusion in the Proceedings, concerning relaxation
} 
deserve however detailed consideration, even for simple designs in which the 3-D magnetic elements are themselves straight, and it is with these issues that we shall be concerned in the present note with the object of giving adequate attention to the influence of the end-winding configurations.

We propose here a form in which it may be convenient to express a general non-skew 3-D magnetic field and we indicate two alternative methods by which we have proceeded to develop such an explicit form as a consequence of field computations based on applications of the Biot and Savart law. It is our hope that the proposed form for expressing the field will prove to constitute an efficient means for transferring to a subsequent user field information concerning the magnetic field under consideration and that this form also will prove convenient for such future dynamical computations as may be required for evaluation of the quality of the field. It moreover is our hope that the procedure we describe will lead to further developments in which such goals can be more accurately and efficiently attained through a more perfectly understood and more sophisticated application of computational techniques akin to those we outline here.

We have developed two methods for computing the functions $A_{n}(z)$ and their derivatives and used them to calculate the magnetic field anywhere inside the curl-free divergence-free region. The first method is using Biot-Savart to compute the magnetic field from a set of "line current" segments that simulate the coil windings. Calculating the magnetic field components at several discrete angles on an arc (e.g. at a 50\% radius of the inner bore) so chosen that a Fourier analysis can conveniently be performed, the harmonic components are evaluated and stored for successive values of $z$. Next these components are numerically differentiated with respect to $z$ ( and hence the name - NUMERICAL method) to provide A values and their derivatives up through the $6^{\text {th }}$ order . In the second method we eliminate the need for harmonic analysis by using the Biot-Savart formula directly (Appendix A) with Differential Algebra ( DA ), yielding both the $A_{n}(z)$ and their derivatives simultaneously. ef. It should be noted that when the magnetic field can be expressed in terms of analytical functions of $Z$ the $A_{n}(z)$ 's and their derivatives can be computed directly as in the simple case where $A_{2}(z)=\cos \left(\frac{\pi z}{L}\right)$ and the solution is separable and reduces to Bessel functions in $r$.

In this report we apply both methods to the "end" region of two magnets - the 50-mm bore dipole D19, and the 40-mm bore SSC quadrupole QC. In the dipole case we computed A1 through $\frac{\partial^{8} A_{1}(z)}{\partial z^{8}}$, and A3 through A9 with up to 6 derivatives each. In the quadrupole case we computed $A 2$ through $\frac{\partial^{16} A_{2}(z)}{\partial z^{16}}, A 6$ through $\frac{\partial^{12} A_{6}(z)}{\partial z^{12}}, A 10$ through $\frac{\partial^{8} A_{10}(z)}{\partial z^{8}}, A 14$ through computations for the field contributions arising from a high-permeability yoke with a circular bore [Caspi, Helm, and Laslett, Lawerence Berkeley Laboratory Report LBL-29826/SC-MAG-317, July 1991].

d The $40 \mathrm{~mm}$ SSC Arc Quadrupole - Magnetic Design - S.Caspi , M.Helm , and L.J. Laslett, SC-MAG-314, LBID_1677, November 1990.

e Differential Algebraic Description of Beam Dynamics to Very High Orders - M.Berz , SSC Central Design Group , Lawrence Berkeley Laboratory

$f$ DAFOR - Differential Algebra Precompiler Version 3, Reference Manual - - M.Berz , Department of Physics and Astronomy and National Superconducting Cyclotron Laboratory, Michigan State University, East Lansing, Mi 48824 
$\frac{\partial^{4} A_{14}(z)}{\partial x^{4}}$, and A18 through $\frac{\partial^{4} A_{18}(z)}{\partial z^{4}}$. In part 2 we have reconstructed the field from the As for the quadrupole magnet and compared results with direct Biot-Savart field calculation. The plots for the A functions themselves are presented in part 3. 


\section{Part 1-3D Field Representation.}

We note that in the curl-free divergence-free region near the axis $\mathrm{r}=0$ the field components may be expressed as given by $\vec{B}=-\nabla V$ where $\mathrm{V}$ is a scalar potential function for which $\nabla^{2} V=0$. The proposed solution ( non-skew ) can be written in the form :

$$
V=\sum_{n=1} V_{n}(r, z) \sin n \theta
$$

with :

$$
\frac{1}{r} \frac{\partial}{\partial r}\left(r \frac{\partial V_{n}}{\partial r}\right)+\frac{\partial^{2} V_{n}}{\partial z^{2}}-\frac{n^{2} V_{n}}{r^{2}}=0
$$

We note that if $V_{n}$ were to be free of any $z$-dependence, the acceptable solution for $V_{n}$ near the axis would be expressed by a single term proportional to $r^{n}$ (i.e., involving $r$ raised to the positive power $n$ ); more generally one would represent $V_{n}$ by a power series involving factors $r^{n+2 k}$, commencing with $r^{n}$, and employing z-dependent coefficients :

$$
V_{n}=\sum_{k=0} C_{n, k}(z) r^{n+2 k}
$$

with $C_{n, k}(z)$ satisfying the recursion relation

$$
C_{n, k}(z)=-\frac{1}{4 k(n+k)} \frac{d^{2} C_{n, k-1}}{d z^{2}} \quad ; \quad k=1, \ldots
$$

The magnetic field components can be derived accordingly as :

$$
\begin{aligned}
& B_{r}=-\sum_{n} \frac{\partial V_{n}}{\partial r} \sin n \theta=\sum_{n} g_{r n} r^{n-1} \sin n \theta \\
& B_{\theta}=-\sum_{n}^{n} \frac{n}{r} V_{n} \cos n \theta=\sum_{n} g_{\theta n} r^{n-1} \cos n \theta \\
& B_{z}=-\sum_{n} \frac{\partial V_{n}}{\partial z} \sin n \theta=\sum_{n} g_{z n} r^{n} \sin n \theta
\end{aligned}
$$

In order that the series for $V_{n}$ satisfy the differential equation written above we introduce $A_{n}(z)$ and express the coefficients $g_{r n}, g_{\theta n}, g_{z n}$ as general functions of $\mathrm{r}$ and $\mathrm{z}$ as shown below :

$$
\begin{aligned}
& g_{r n}(r, z)=\sum_{k=0}(-1)^{k+1} \frac{n !(n+2 k)}{2^{2 k} k !(n+k) !} A_{n}^{(2 k)}(z) r^{2 k} \\
& g_{\theta n}(r, z)=\sum_{k=0}(-1)^{k+1} \frac{n ! n}{2^{2 k} k !(n+k) !} A_{n}^{(2 k)}(z) r^{2 k} \\
& g_{z n}(r, z)=\sum_{k=0}(-1)^{k+1} \frac{n !}{2^{2 k} k !(n+k) !} A_{n}^{(2 k+1)} r^{2 k}
\end{aligned}
$$


Explicitly we can write the above as :

$$
\begin{gathered}
g_{r n}(r, z)=-n A_{n}(z)+\frac{n+2}{4(n+1)} A_{n}^{\prime \prime}(z) r^{2}-\frac{n+4}{32(n+1)(n+2)} A_{n}^{\prime \prime \prime \prime}(z) r^{4} \\
+\frac{n+6}{384(n+1)(n+2)(n+3)} A_{n}^{\prime \prime \prime \prime \prime}(z) r^{6}-\ldots \\
g_{\theta n}(r, z)=-n A_{n}(z)+\frac{n}{4(n+1)} A_{n}^{\prime \prime}(z) r^{2}-\frac{n}{32(n+1)(n+2)} A_{n}^{\prime \prime \prime \prime}(z) r^{4} \\
+\frac{n}{384(n+1)(n+2)(n+3)} A_{n}^{\prime \prime \prime \prime \prime \prime}(z) r^{6}-\ldots \\
g_{z n}(r, z)=-A_{n}^{\prime}(z)+\frac{1}{4(n+1)} A_{n}^{\prime \prime \prime}(z) r^{2}-\frac{1}{32(n+1)(n+2)} A_{n}^{\prime \prime \prime \prime \prime}(z) r^{4} \ldots .
\end{gathered}
$$

We have computed $g_{m}, g_{\theta n}$ and $g_{z n}$ from which we calculated $A(z)$ and derivatives (part 3). In general the need for high derivatives and their relative contribution to the magnetic field should be considered only when field values are needed close to the winding.

\section{$\underline{\text { Extended }}=\underline{\text { 3D }}$ Field.}

A more general form for the scalar potential of a 3D magnetic field interior to the windings includes both "skew" and "non-skew" terms of all integer harmonic of order $n$ (including $n=0$ ):

$$
V=-\left\{\sum_{n=0} r^{n} \sum_{k=0} \frac{(-1)^{k+1} n !}{2^{2 k} k !(n+k) !} r^{2 k}\left[A_{n}^{(2 k)}(z) \sin n \theta-\tilde{A}_{n}^{(2 k)}(z) \cos n \theta\right]\right\}
$$

for which $\nabla^{2} V=0$ ( the negative sign in front of the "skew" term complies with the right hand rule).

The magnetic field can be derived accordingly as :

$$
\begin{aligned}
& B_{r}=-\frac{\partial V}{\partial r}=\sum_{n}\left[g_{r n} r^{n-1} \sin n \theta-\tilde{g}_{r n} r^{n-1} \cos n \theta\right] \\
& B_{\theta}=-\frac{n}{r} V=\sum_{n}\left[g_{\theta n} r^{n-1} \cos n \theta+\tilde{g}_{\theta n} r^{n-1} \sin n \theta\right] \\
& B_{z}=-\frac{\partial V}{\partial z}=\sum_{n}\left[g_{z n} r^{n} \sin n \theta-\tilde{g}_{z n} r^{n} \cos n \theta\right]
\end{aligned}
$$

where

$$
\begin{aligned}
& g_{r n}=\tilde{g}_{r n} \\
& g_{\theta n}=\tilde{g}_{\theta n} \\
& g_{z n}=\tilde{g}_{z n}
\end{aligned}
$$

are general functions of $\mathrm{r}$ and $\mathrm{z}$ that include the appropriate "non-skew" and "skew" terms $A_{n}(z)$ and $\tilde{A}_{n}(z)$. 


\section{Integral Form.}

In commenting on a power series development in terms of powers of $\mathrm{r}$ of the form teated above, Lloyd Smith has referred to an earlier HIFAR Note-167 when remarking (in HIFAR Note-173) that a potential function of harmonic order $\mathrm{n}$, such as the function $V_{n}(r, z)$ introduced here, can be expressed more compactly in terms of an integral - thus it is stated that:

For a function $V_{n}(r, z)_{\cos n \theta}^{\sin n \theta}$ that is of the form $\left(r^{n} A_{n}(z)+\text { terms of higher order in } r\right)_{\cos n \theta}^{\sin n \theta}$ and where $\sin n \theta$ and $\cos n \theta$ denote "non-skew" and "skew" terms, one may write

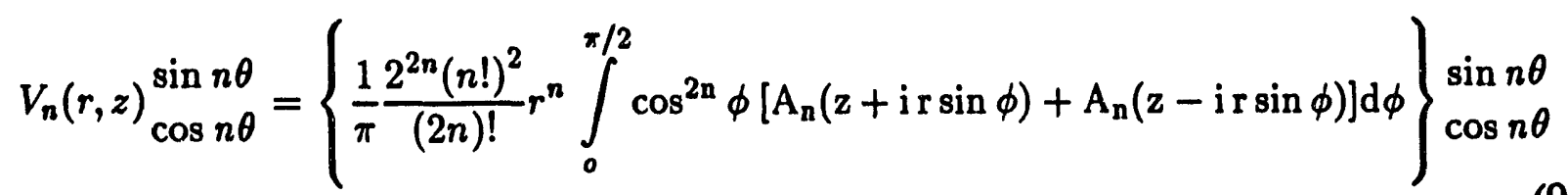

$$
\begin{gathered}
=\left\{\frac{1}{\pi} \frac{2^{2 n}(n !)^{2}}{(2 n) !} r^{n}\left[2 \sum_{k=0} \frac{(-1)^{k}}{(2 k) !} r^{2 k} \int_{0}^{\pi / 2} \cos ^{2 n} \phi \sin ^{2 k} \phi \mathrm{d} \phi\right] A_{n}^{(2 k)}(z)\right\} \begin{array}{c}
\sin n \theta \\
\cos n \theta
\end{array} \\
=\left\{\frac{1}{\pi} \frac{2^{2 n}(n !)^{2}}{(2 n) !} r^{n}\left[\sum_{k=0} \frac{(-1)^{k} r^{2 k}}{(2 k) !} \frac{(2 n) !(2 k) !}{2^{2(n+k)} n ! k !(n+k) !} \pi\right] A_{n}^{(2 k)}(z)\right\} \begin{array}{l}
\sin n \theta \\
\cos n \theta
\end{array} \\
=r^{n} \sum_{k=0}(-1)^{k} \frac{n !}{2^{2 k} k !(n+k) !} r^{2 k} A_{n}^{(2 k)}(z) \begin{array}{c}
\sin n \theta \\
\cos n \theta
\end{array} \\
=r^{n} \sum_{k=0} C_{n, k}(z) r^{2 k} \sin n \theta \\
\cos n \theta
\end{gathered}
$$

with

$$
C_{n, k}(z)=\frac{(-1)^{k} n !}{2^{2 k} k !(n+k) !} A_{n}^{(2 k)}(z)
$$

then providing the desired form with Laplacian equal to zero as was introduced on p.2. [Dr. Smith has verified (HIFAR Note-173, p.2) that use of the integral form shown above leads to a potential function with zero Laplacian.]

Note: The integral

$$
\begin{gathered}
\Lambda \equiv 2 \int_{0}^{\pi / 2} \cos ^{2 n} \phi \sin ^{2 k} \phi d \phi \\
=\mathrm{B}\left(n+\frac{1}{2}, k+\frac{1}{2}\right)(\text { See Gradshteyn and Ryzhik p.369 sect } 3.621(5)) \\
=\frac{\Gamma\left(n+\frac{1}{2}\right) \Gamma\left(k+\frac{1}{2}\right)}{\Gamma(n+k+1)}(\text { See ditto p.350 sect } 8.384(1))
\end{gathered}
$$


where B is the Beta function (Euler's integral of the first kind). But

Accordingly

$$
\Gamma\left(n+\frac{1}{2}\right)=\frac{(2 n) !}{2^{2 n} n !} \sqrt{\pi}(\text { See ditto } p .938 \text { sect } 8.339(2)) \text {. }
$$

$$
\Lambda=\frac{(2 n) !(2 k) ! \pi}{2^{(n+k)} n ! k !(n+k) !}
$$

\section{The Case $n=0$}

As a special case we write down the field components for $n=0$ :

$$
\begin{aligned}
& B_{r}=-\sum_{k=0}(-1)^{k+1} \frac{k}{k ! k !} \tilde{A}_{0}^{(2 k)}(z)\left(\frac{r}{2}\right)^{2 k-1} \\
& B_{\theta}=0 \\
& B_{z}=-\sum_{k=0}(-1)^{k+1} \frac{1}{k ! k !} \tilde{A}_{0}^{(2 k+1)}(z)\left(\frac{r}{2}\right)^{2 k}
\end{aligned}
$$

or explicitly :

$$
\begin{aligned}
& B_{r}=0-\tilde{A}_{0}^{\prime \prime}(z)\left(\frac{r}{2}\right)+\frac{1}{2} \tilde{A}_{0}^{\prime \prime \prime \prime}(z)\left(\frac{r}{2}\right)^{3}-\frac{1}{12} \tilde{A}_{0}^{\prime \prime \prime \prime \prime}(z)\left(\frac{r}{2}\right)^{5} \ldots \\
& B_{\theta}=0 \\
& B_{z}=\tilde{A}_{0}^{\prime}(z)-\tilde{A}_{0}^{\prime \prime \prime}(z)\left(\frac{r}{2}\right)^{2}+\frac{1}{4} \tilde{A}_{0}^{\prime \prime \prime \prime}(z)\left(\frac{r}{2}\right)^{4} \ldots
\end{aligned}
$$

Note that:

$$
\frac{\partial B_{r}}{\partial z}=\frac{\partial B_{z}}{\partial r}
$$

The form proposed above in which a 3-D field throughout an interior region is expressed in terms of the function $\tilde{A}_{0}(z)$ and its derivatives may appear analogous in spirit to the familiar similar description of fields with rotational symmetry in terms of a z-directed field ( solenoid ) $\tilde{A}_{0}^{\prime}(z)=B_{0, z}(z)$ and its derivatives along the symmetry axis.?

The representation specified above for 3-D magnetic fields, written in terms of functions $A_{n}(z)$ and $\tilde{A}_{n}(z)$ and their derivatives, will describe a field that formally is both divergence free and curl free - provided that the summations are not truncated. If, however, we wish to truncate these series expressions, we at best can only do so in such a way that one, but not both, of these conditions is satisfied. Thus, if we wish to preserve the divergence condition $\nabla \cdot \overrightarrow{\mathbf{B}}=0$ (so that the consequent dynamical equations for charged-particle motion can be derived from a Hamiltonian function in which the magnetic field is described by a vector potential), we should take care that the sum over the index $k$ (e.g. in Eqs. 4 or 20 above) in the series for $B_{z}$ should terminate at a value of $k$ that is less by unity than the termination value for this index in the series for the transverse field components $B_{r} \& B_{\theta}$. One possible form for the vector potential of such a field is indicated in Appendix A (p.41) an LBL report ${ }^{h}$ relating to a non-skew 3-D field. See V. E. Cosalech, "Introduction to Electron Optics" (Oxford Univ. Press, Ed. 2, 1950), 136, esp. Eqns. (IV.45) and (IV.46), p.106 UC-405/LBL-30313/SC-MAG-328 


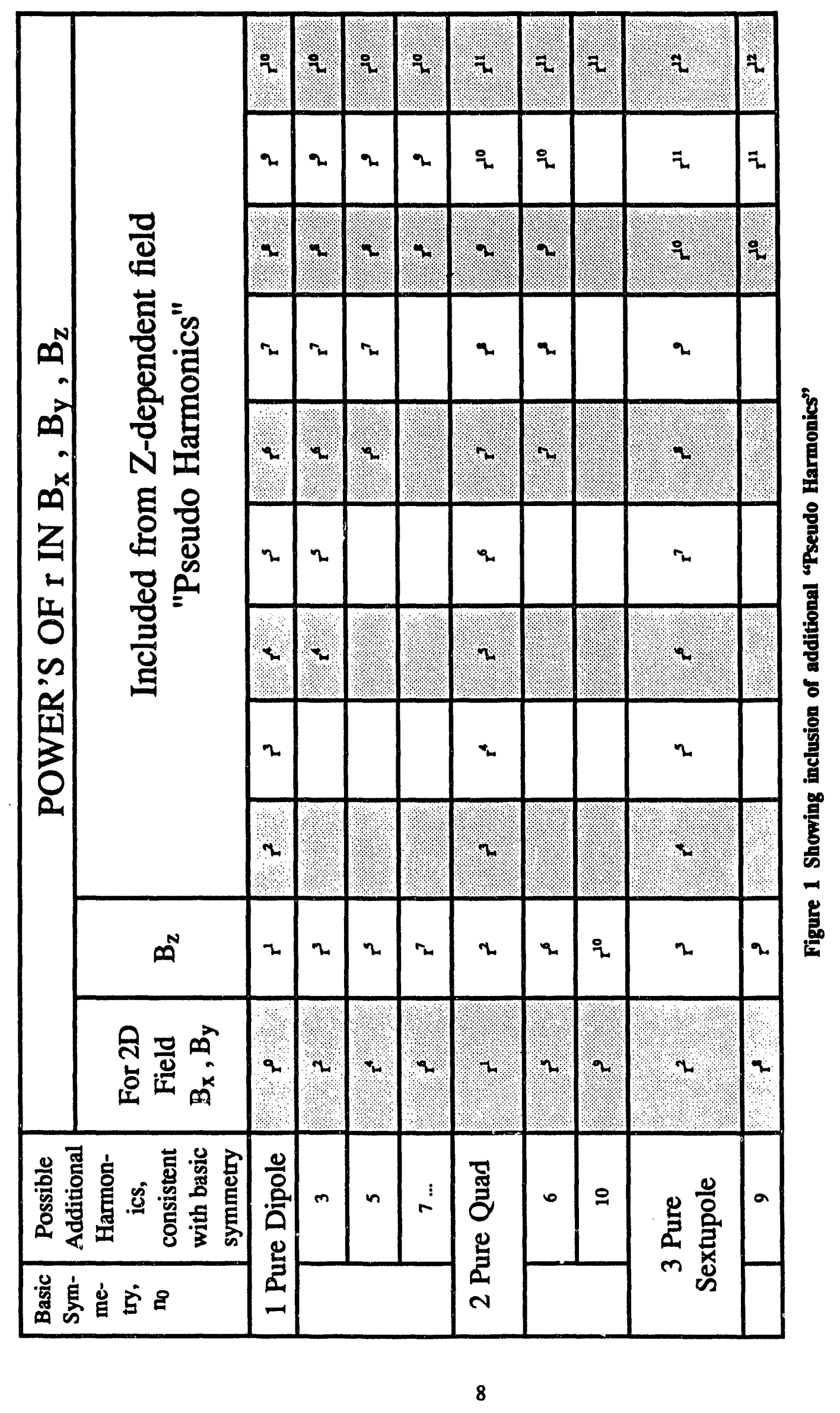




\section{The "Biot \& Savart" Field Evaluation}

The current windings of a typical magnet element may be regarded as formed of "cables" for which the resuling field may be computed, in the case of complete curcuits, through use of the law of Biot \& Savart or Ampere: $\vec{B}=\frac{\mu_{0}}{4 \pi} \oint \frac{J \vec{d} l \vec{r}_{c \rightarrow p}}{r^{3}}$ (with $\mu_{0}=\frac{4 \pi}{10}$ for cm, amp, and gauss; $4 \pi \times 10^{-7}$ for meter, amp, and Tesla). In application, to accommodate curved and twisted cables it appears desirable to break the individual cables into a number of short "blocks", with quadrilateral (planar) end-faces (A \& B) and to calculate the individual contributions of these several blocks to the magnetic field.

For blocks of significant cross-section the field calculations are based on representing the block current by that of several straight "wires", extending between the end faces, in such a manner that (because of transposition of the actual conductors within a superconducting cable) a constant current density across every transverse cross-section will be represented even if the cable itself is curved. If local coordinate variables $\xi, \eta$ are used to denote the wire end-points in the end faces A \& B (with $-1<\xi<1,-1<\eta<1$ ), it may be economical computationally, when computing fields at substantial distances from a current block, to situate such wires at $\xi, \eta$ locations appropriate for 2-D Gaussian integration with wire currents proportional to their corresponding Gaussian weighting factors; for computing field contributions at locations very close to a current block, however, it can be preferable to employ a number of wires consistent with the number (e.g., $2 \times 18$ ) of which the cable is actually formed and to situate these wires (with equal weights) uniformly across the cross-section of the cu rent block.

The "Biot \& Savart" contribution of an individual straight wire segment (extending between $x_{A}, y_{A}, z_{A}$ and $\left.x_{B}, y_{B}, z_{B}\right)$ to the field at a field-point location $\mathrm{X}, \mathrm{Y}, \mathrm{Z}$ is given by the formula written below (for $B_{Y}$, with other components similarly represented by cyclical premutation).

Define $x_{m}=\frac{x_{A}+x_{B}}{2}$, etc.; $\Delta x=\frac{x_{B}-x_{A}}{2}$, etc.; and

$$
\begin{aligned}
& T 1=\left(y_{M}-Y\right) \Delta z-\left(z_{M}-Z\right) \Delta y \\
& T 2=\left(z_{M}-Z\right) \Delta x-\left(x_{M}-X\right) \Delta z \\
& T 3=\left(x_{M}-X\right) \Delta y-\left(y_{M}-Y\right) \Delta x \\
& T A=\left(x_{A}-X\right) \Delta x+\left(y_{A}-Y\right) \Delta y+\left(z_{A}-Z\right) \Delta z \\
& T B=\left(x_{B}-X\right) \Delta x+\left(y_{B}-Y\right) \Delta y+\left(z_{B}-Z\right) \Delta z \\
& D A F=\left[\left(x_{A}-X\right)^{2}+\left(y_{A}-Y\right)^{2}+\left(z_{A}-Z\right)^{2}\right]^{1 / 2} \\
& D B F=\left[\left(x_{B}-X\right)^{2}+\left(y_{B}-Y\right)^{2}+\left(z_{B}-Z\right)^{2}\right]^{1 / 2} \\
& D M S=(T 1)^{2}+(T 2)^{2}+(T 3)^{2} .
\end{aligned}
$$

Then

$$
\begin{aligned}
B_{Y}=-T 2 \frac{\mu_{o}}{4 \pi} I \int_{-1}^{1} & \frac{d t}{\left\{\left[\left(X-x_{M}\right)-t \Delta x\right]^{2}+\left[\left(Y-y_{M}\right)-t \Delta y\right]^{2}+\left[\left(Z-z_{M}\right)-t \Delta z\right]^{2}\right\}^{3 / 2}} \\
& =\frac{\mu_{o}}{4 \pi} I\left[\left(\frac{T 2}{D B F}\right)\left(\frac{T B}{D M S}\right)-\left(\frac{T 2}{D A F}\right)\left(\frac{T A}{D M S}\right)\right] .
\end{aligned}
$$


Such formulas form the basis of a routine GAUSBLK. For numerical (arithmetic) computation it may be advisable to introduce some "scaling" of distance parameters, introducing a common scale factor into $\Delta x, \Delta y, \Delta z, D A F$, and DBF (thus automatically also scaling $T 1, T 2$, and T3) and further scaling once more TA and TB (so that ultimately TA, TB, and. DMS become doubly scaled). [Ideally the values obained for the field components should not be modified by this scaling.] ( see Appendix A for a computer listing ).

An especially detailed application of GAUSBLK may be needed for evaluation of fields at the edge, or in the interior (near the individual wires), of a superconducting cable in order to assess the danger of reaching quenching conditions. In other applications one will only require field evaluations at somewhat more remote locations in order (i) to determine harmonic components of the direct field near surrounding polarizable material ${ }^{i}$ or (ii) to enable proceeding with field analyses of the type required to evaluate coefficients such as the functions $A_{n}(z)$ (and their derivatives) introduced on p. 2. We outline below two methods that may be used to perform, in the region interior to the windings, the field analyses mentioned immediately above - namely an algebraic finite-difference method and, as an alternative, the use of the differential-algebra (D-A) routine developed by M. Berz.j

\section{A Numerical Finite-Difference Method}

This method was proven to be more successful than originally thought. We have used the Biot-Savart relation in conjunction with a large number of current line elements to compute the field at a given point. The file containing the coil geometry consists of 52 conductor blocks per turn with $2 \times 8$ (or more) line currents per block in a minimum symmetry configuration. However, during computation the program generated the line current for all other symmetries (e.g. $\times 4$ for a dipole, $x 8$ for a quad).

In use of this method the GAUSBLK routine is used to determine numerically the spatial components of magnetic field at many $z$ locations, several azimuthal locations, and (in our particular application) a single interior value of the polar-coordinate radius (e.g., $r=1.0$ ). These numerical data are subjected to Fourier analysis vs. $\theta$ so as to obtain tabular values, for each apparent non-vanishing harmonic, of the functions $g_{r, n}, g_{\theta, n}$, and $g_{z, n}$ defined by Equations 3-4 (if only "non-skew" elements are present) and in which we henceforth may for brevity at times drop the subscripts $\mathrm{n}$. [It may be noted that, in cases of basic dipole symmetry, quadrupole symmetry, or the like, the Fourier analysis need be performed solely in a single quadrant, octant, or so forth and can be particularly economically performed if the $\theta$ values adopted are equally spaced (to take advantage of the orthogonality that such circular functions will then present with respect to summation). One may need, however, to take care to avoid the development of significant errors from the phenomenon of "aliasing" when only a limited number of $\theta$ locations is employed in the Fourier analyses.] As will also be the case in an economical use of the D-A procedure one thus can perform the Fourier analysis, once the intended basic (non-skew) symmetry $n_{o}$ is presumed, solely on the field of (closed) windings in the interval $0<\theta<\frac{\pi}{2 n_{0}}$. In this case the algebraic Fourier analysis need be performed solely for values of $n=n_{o}(2 l-1)$, with $l=1,2, \cdots$, and one then multiplies the resultant values of $g_{r, n}, g_{\theta, n}$, and $g_{z, n}$ each by $4 n_{0}$.

As treated by V. Brady, in HIFAR Note-261 (LBL, Jan 26, 1990)

Particle Accelerator 24, 109 (1989 
For each relevant harmonic number (n) the functions $g_{r}, g_{\theta}$, and $g_{z}$ are regarded as expressable in terms of the functions $A_{n}(z)$ as [Eq. 5] in which superscripts (k) on A denote $\frac{d^{k}}{d z^{k}} A(z)$ and the subseript $\mathrm{n}$ has been dropped in the interest of brevity. It is the task of a routine such as AVALS to derive from values of $g_{r}, g_{\theta}$, and $g_{z}$ at a single radius $(r)$ and several adjacent $z$ locations, values of $A(z)$ and of a few derivatives of $A$ that will serve so to specify the magnetic field in the interior region. We proceed to illustrate here how this specifically may be done (version 6 of the "outine AVALS) by use of numerical values of $g_{r}$ and $g_{\theta}$ at a "central" location $\left(z_{0}\right)$ and values of $g_{x}$ at this central location and at 3 additional equally-spaced values of $z$ to each side. [We note from the equations wrtten above that under conditions in which the $\mathrm{z}$ dependence of the magnetic field becomes small the functions $g_{r}$ and $g_{\theta}$ tend to become identical (and the function $g_{z}$ becomes small); it is for this reason that, in the procedure to be described kelow, we consider it desirable to make use of the values of $g_{z}$ at several adjacent $z$ locations and to employ only a single (suitably-weighted) average of $g_{r}, g_{\theta}$.]

To continue with this illustration in which we estimate the function $A\left(z_{0}\right)$, through use of values of $g_{r}\left(z_{0}\right)$ and $g_{\theta}\left(z_{0}\right)$ and values of $g_{z}\left(z_{0} \mp 3 h\right), g_{z}\left(z_{0} \mp 2 h\right), g_{z}\left(z_{0} \mp h\right)$, and $g_{z}\left(z_{0}\right)$, we presume that a Taylor-series development in this neighborhood can ignore derivatives of order 7 and greater. With the definitions

$$
\alpha=\frac{r^{2}}{4(n+1)}, \beta=\frac{r^{4}}{32(n+1)(n+2)}
$$

we then write

$$
\begin{gathered}
g_{z}\left(z_{0}\right)=-A^{\prime}\left(z_{0}\right)+\alpha A^{\prime \prime \prime}\left(z_{0}\right)-\beta A^{v}\left(z_{0}\right) \\
D_{1}\left(z_{0}\right):=\frac{g_{z}\left(z_{0}-h\right)-2 g_{z}\left(z_{0}\right)+g_{z}\left(z_{0}+h\right)}{h^{2}}=-A^{\prime \prime \prime}\left(z_{0}\right)+\left[\alpha-\frac{h^{2}}{12}\right] A^{v}\left(z_{0}\right)
\end{gathered}
$$

and

$$
D_{2}\left(z_{0}\right):=\frac{g_{z}\left(z_{0}-2 h\right)-2 g_{z}\left(z_{0}\right)+g_{z}\left(z_{0}+2 h\right)}{4 h^{2}}=-A^{\prime \prime \prime}\left(z_{0}\right)+\left[\alpha-\frac{h^{2}}{3}\right] A^{v}\left(z_{0}\right) .
$$

From these equations it then follows that

$$
\begin{aligned}
& A^{\prime}\left(z_{0}\right)=-g_{z}\left(z_{0}\right)+4\left[\frac{\alpha^{2}-\beta}{h^{2}}-\frac{\alpha}{3}\right] D_{1}\left(z_{0}\right)+\left[\frac{\alpha}{3}-\frac{4\left(\alpha^{2}-\beta\right)}{h^{2}}\right] D_{2}\left(z_{0}\right) \\
& A^{\prime \prime \prime}\left(z_{0}\right)=4\left[\frac{\alpha}{h^{2}}-\frac{1}{3}\right] D_{1}\left(z_{0}\right)+\left[\frac{1}{3}-\frac{4 \alpha}{h^{2}}\right] D_{2}\left(z_{0}\right) \\
& A^{v}\left(z_{0}\right)=\frac{4}{h^{2}} D_{1}\left(z_{0}\right)-\frac{4}{h^{2}} D_{2}\left(z_{0}\right) .
\end{aligned}
$$

From analogous definitions of $D_{1}\left(z_{0} \mp h\right)$ and of $D_{2}\left(z_{0} \mp h\right)$ one likewise can obtain values of the odd derivatives $A^{\prime}\left(z_{0} \mp h\right), A^{\prime \prime \prime}\left(z_{0} \mp h\right)$, and $A^{\prime \prime}\left(z_{0} \mp h\right)$. Such values then permit one to 
evaluate the even derivatives at $z_{0}$ with similar accuracy, viz:

$$
\begin{aligned}
& A^{v i}\left(z_{0}\right)=\frac{A^{v}\left(z_{0}+h\right)-A^{v}\left(z_{0}-h\right)}{2 h} \\
& A^{i v}\left(z_{0}\right)=\frac{A^{\prime \prime \prime}\left(z_{0}+h\right)-A^{\prime \prime \prime}\left(z_{0}-h\right)}{2 h}-\frac{h^{2}}{6} A^{v i}\left(z_{0}\right) \\
& A^{\prime \prime}\left(z_{0}\right)=\frac{A^{\prime}\left(z_{0}+h\right)-A^{\prime}\left(z_{0}-h\right)}{2 h}-\frac{h^{2}}{6} A^{i v}\left(z_{0}\right)-\frac{h^{4}}{120} A^{v i}\left(z_{0}\right) .
\end{aligned}
$$

Finally to obtain the functicn $A\left(z_{0}\right)$ itself we employ a suitable average of the quantities $g_{r}\left(z_{0}\right)$ and $g_{\theta}\left(z_{0}\right)$, an average that in this example we take to be

$$
G D:=n g_{r}\left(z_{0}\right)-(n+6) g_{\theta}\left(z_{0}\right)
$$

To the accuracy sought here this form may be expressed as

$$
\left.G D \approx 6 n A\left(z_{0}\right)-\frac{n}{n+1} r^{2} A^{\prime \prime}\left(z_{0}\right)+\frac{n}{16(n+1)(n+2)} r^{4} A^{i(i)} z_{0}\right)
$$

since the relative weighting factors proposed for forming this average are such as to eliminate the derivative $A^{v i}\left(z_{0}\right)$ that may have been determined to only moderate accuracy. In the procedure outlined here we accordingly take the function $A\left(z_{0}\right)$ to be given by

$$
A\left(z_{0}\right)=\frac{G D+\frac{n}{n+1} r^{2} A^{\prime \prime}\left(z_{0}\right)-\frac{n}{16(n+1)(n+2)} r^{4} A^{i v}\left(z_{0}\right)}{6 n} .
$$

Analogous procedures, of various expected accuracies, can be similarly designed to emplcy differing numbers of $g_{z}(z)$ values in the neighborhood of a "central" $z_{0}$ and the arithmetical correctness of such procedures can be verified by tests in which (for example) the function $A(z)$ is taken to be such a function as $A(z)=[2(z+1)]^{N Z-1}$ for which derivatives of order NZ and beyond vanish identically. It should be noted that the procedures specifica'ly described here require the availability of values of $g_{r}, g_{\theta}$, and $g_{z}$ at only a single value of the coordinate $r$ and that appropriate use of such procedures may require some judgment be exensised in selecting the value of this radial coordinate to be employed in such work.

The numerical quality of the As was proven to be of high accuracy especially in the vicinity of the radius at which the Fourier analysis was performed. This is not a surprise however, but it does restrict somewhat the region of interpolation. On the other hand this method provides the means for calculating fields closer to the windings by choosing a proper radius. It should be noted that this method is independent of which method is used in calculating the magnetic field and therefore can be applied to problems with iron. If the iron is allowed to saturate the As become a function of the current and therefore more complicated. 


\section{Differential Algebra Method}

It appears possible in principle to proceed directly, through use of the Differential Algebra program developed by $M$. Berz, from a fomula representing a Biot and Savart evaluation of $B_{Y}\left(B_{\theta}\right.$ at $\left.\theta=0\right)$ to evaluation of the functions $A_{n}^{(k)}(z)$ that may serve to describe the field in the manner shown by Eq. 3-4. In particular one may by this means (i) avoid the need to make an initial preliminary Fourier analysis of the field components (in the manner outlined by the Numerical method ) and (ii) avoid the need to approximate various derivatives of functions $A_{n}(z)$ through use of finite- difference forms applied fo field components at some non-zero radius.

We may remark that the magnetic field formed from functions $g_{r}, g_{\theta}$, and $g_{z}$ of the type written for the Jumerical method should, if not truncated, suitably represent a field that is both diverenge free and curl free - as should be the case for a field derived by application of the Biot and Savart law to a closed circuit. ${ }^{k}$ Under such conditions it may be permissible to undertake evaluation of the functions $A_{n}(z)$ through a somewhat curtailed use of the forms shown previously. We accordingly outline below a procedure based on use of a Biot and Savart expression for the field of a (non-skew) current distribution at field points $(X, Z)$ in the median plane $(\theta=0, Y=0)$ - presuming that this expression is legitimately differentiable (several times) with respect to the field-point coominates $X$ and $Z$ (including cross derivatives). We indicate below [case A] a method whereby direct applications of the D-A procedure can in principle be used to obtain tabulations of the fuctions $A_{n}^{(k)}(z)$ and [case B] an alternative method that more economically may be used in situations for which symmetry permits the Biot and Savart formulas to be written explicitly (but in a closed-circuit form) for only a portion of the interval $2 \pi$ in $\theta$. In either case, as also in the Numerical method, a periodic array of course will require recognition of at least one or two magnet element neighbors (possibly of alternating sign) on either side of a central magnet element.

case A Such a direct use of the differential algebra (D-A) procedure can be conveniently illustrated by consideration of a fully described current winding with a basic non-skew quadrupole symmetry. In this case the $\theta$ dependence of the magnetic fields will be characterized by harmonic indices $n$ equal to odd multipiles of $n_{o}$ with $n_{o}=2$ - namely $n=2$ (quadrupole), $n=6$ (dodecapole), etc., and for which we write explicitly below only the first few terms of a development based on a representation of the magnetic field consistent with Eq. 3-5 :

$$
\begin{aligned}
\left.\left.B_{\theta}\right|_{\theta=0} \equiv B_{Y}\right|_{Y=0}=\left[-2 X A_{2}(Z)+\frac{1}{6} X^{3} A_{2}^{\prime \prime}(Z)\right. & -\frac{1}{192} X^{5} A_{2}^{i v}(Z)+\cdots \\
& \left.-6 X^{5} A_{6}(Z)+\cdots\right]
\end{aligned}
$$

For this development to be of a valid form, all octants should be filled with wire configurations of the equivalent non-skew type -or, if only the upper octants are so filled, the lower octants should be considered also filled in the same manner and the functions $A_{n}^{(k)}(z)$, where $\mathrm{n}$ is an odd multiple of $n_{o}$, derived by the procedure outlined below then should be doubled. It is evident that if one forms the first derivative of this expression with respect to the variable $\mathbf{X}$

As mentioned, the functions $g_{r}, g_{\theta}$, and $g:$ can be truncated in such a manner as to be at least divergemce free and such a field accordingly could be regarded as derived from a vector potential. 
and evaluates this at $\mathrm{X}=0$ one obtains

$$
\left[\frac{\partial}{\partial X}\left[\left.B_{Y}\right|_{Y=0}\right]\right]_{X=0}=-2 A_{2}(Z)
$$

or

$$
A_{2}(Z)=-\frac{1}{2}\left[\frac{\partial}{\partial X}\left[\left.B_{Y}\right|_{Y=0}\right]\right]_{X=0}
$$

and further differentiation with respect to $\mathrm{Z}$ will then similarly provide various derivatives of the function $A_{2}(Z)$ with respect to its argument.

Information concerning the function $A_{6}(Z)$ similarly is obtainable by evaluating the fifth derivative of $\left.B_{Y}\right|_{Y=0}$ with respect to $\mathrm{X}$ at $\mathrm{X}=0$, save that one will have to take into account a contribution from the quadrupole term $A_{2}^{i v}$ - thus:

$$
\left[\frac{\partial^{5}}{\partial X^{5}}\left[\left.B_{Y}\right|_{Y=0}\right]\right]_{X=0}=-\frac{5 !}{192} A_{2}^{i v}(Z)-6 \times(5 !) A_{6}(Z)
$$

or

$$
\begin{aligned}
A_{6}(Z) & =-\frac{\left[\left.\frac{\partial^{5}}{\partial X^{5}} B_{Y}\right|_{Y=0}\right]_{X=0}+\frac{5 !}{192} A_{2}^{i v}(Z)}{6 \times 5 !} \\
& =-\frac{1}{720}\left[\left.\frac{\partial^{5}}{\partial X^{5}} B_{Y}\right|_{Y=0}\right]_{X=0}+\frac{1}{2304}\left[\left.\frac{\partial^{5}}{\partial X \partial Z^{4}} B_{Y}\right|_{Y=0}\right]_{X=0} \\
& =-\frac{1}{6} b_{5,0}+\frac{1}{96} b_{1,4},
\end{aligned}
$$

wherein we have introduced the notation

$$
b_{n, m}=\left.\frac{1}{n ! m !} \frac{\partial^{n+m}}{\partial X^{n} \partial Z^{m}} B_{Y}\right|_{Y=0}
$$

at $X=0$ and specific $Z$. [The quantities $b_{n, m}$ introduced here as a convenient notation are identical to quantities that appear as direct output from the version of the D-A program available to us.]

The method of employing the D-A program, as this method has been outlined on the previous page, is readily generalized to cover configurations of other basic harmonic character $\left(n_{0}\right)$ and to extensions to series involving a greater number of derivatives of the functions $A_{n}(Z)$. The algebra that is involved in interpreting D-A output of this nature can be checked if desired, by trial runs involving (for example) a $n_{0}=2$ case in which

$$
\begin{aligned}
\left.B_{Y}\right|_{Y=0} & =c_{2,0} X \cdot A_{2}(Z)+\cdots+c_{2,8} X^{17} \cdot A_{2}^{(16)}(Z) \\
& +c_{6,0} X^{5} \cdot A_{6}(Z)+\cdots+c_{6,6} X^{17} \cdot A_{6}^{(12)}(Z) \\
& +\cdots \\
& +c_{18,0} X^{17} \cdot A_{18}(Z)
\end{aligned}
$$

wherein

$$
c_{n, k}=\frac{(-1)^{k+1} n}{2^{2 k} k !(n+k) ! / n !}
$$


and we elect to choose functions $A_{n}(Z) \propto Z^{18-n}$ (for example $A_{2}(Z)=Z^{16}, A_{6}(Z)=0.9 Z^{12}$, $A_{10}(Z)=0.8 Z^{8}, A_{14}(Z)=0.7 Z^{4}$, and $\left.A_{18}(Z)=0.6\right)$ with evaluations of the $A_{n}(Z)$ and their derivatives sought for (say) $Z=1.0$ through use of the program. Such checks may serve not only to detect possible error in formulating details of the program that is to be subjected to D-A analysis but also may reveal possible inaccuracies that might arise from inadequate register lengths or similar restrictions that become significant during execution.

case B As a more economical method for application of Biot and Savart results, one may employ (in our quadrupole example) only the Biot and Savart formula for (closed) windings in the single octant \#1 - but with the understanding that all octants ultimately will be so filled as to produce a basic quadrupole field ( $n=$ odd multiples of $\left.n_{0}=2\right)$. We then , in submittal to the D-A program, need however to include in the initial field development octupole terms involving functions $A_{4}^{(k)}$, decapole terms $A_{10}^{(k)}$, etc. and to include recognition of such functions in our initial evaluation of the $A_{n}^{(k)}$ - thus:

$$
\begin{aligned}
\left.\left.B_{\theta}\right|_{\theta=0} \equiv B_{Y}\right|_{Y=0}=\left[-2 X A_{2}(Z)\right. & +\frac{1}{6} X^{3} A_{2}^{(2)}(Z)-\frac{1}{192} X^{5} A_{2}^{(4)}(Z)+\cdots \\
& -4 X^{3} A_{4}(Z)+\frac{1}{5} X^{5} A_{4}^{(2)}(Z)-\cdots \\
& \left.-6 X^{5} A_{6}(Z)+\cdots\right]
\end{aligned}
$$

One then forms

$$
b_{1,0}=-2 A_{2}(Z) \text { or } A_{2}(Z)=-\frac{1}{2} b_{1,0}
$$

and thence $A_{2}^{(2)}(Z)=-b_{1,2}, A_{2}^{(4)}(Z)=-12 b_{1,4}$, etc. (including also odd derivatives);

$$
\begin{aligned}
& b_{3,0}=\frac{1}{6} A_{2}^{(2)}(Z)-4 A_{4}(Z) \text { or } A_{4}=\frac{1}{24} A_{2}^{(2)}(Z)-\frac{1}{4} b_{3,0} \\
& =-\frac{1}{24} b_{1,2}-\frac{1}{4} b_{3,0}
\end{aligned}
$$

and thence the derivatives $A_{4}^{(k)}$ including $A_{4}^{(2)}=-\frac{1}{2} b_{1,4}-\frac{1}{2} b_{3,2}$;

$$
b_{5,0}=-\frac{1}{192} A_{2}^{(4)}(Z)+\frac{1}{5} A_{4}^{(2)}(Z)-6 A_{6}(Z)
$$

or

$$
\begin{aligned}
A_{6}(Z) & =\frac{1}{30} A_{4}^{(2)}(Z)-\frac{1}{1152} A_{2}^{(4)}(Z)-\frac{1}{6} b_{5,0} \\
& =-\frac{1}{160} b_{1,4}-\frac{1}{60} b_{3,2}-\frac{1}{6} b_{5,0}
\end{aligned}
$$

and the corresponding $A_{6}^{(k)}$. On completion of such provisional evaluations one then can set identically to zero the functions $A_{4}^{(k)}$, etc., that would be absent if the basic symmetry were present and take the remaining functions (and their derivatives) eightfold to represent the complete quadrupole field with its true harmonics.

Remarks: 
The methods illustrated above by application to a basically quadrupole winding configuration have their corresponding application to any other type of winding but in cases of practical interest may again be supposed to be based on a $\left.B_{Y}\right|_{Y=0}$ development that either required only odd powers of $\mathbf{X}$ or, alternatively, only even powers of $\mathbf{X}$ (including $\mathbf{X}^{0}$ in a development applicable to a dipole).

It may be prudent at this stage of the work to mention a few instances in which some judgment may be required in application of the methods outlined above. Thus, as has already been noted in connection with the section concerning use of the Biot and Savart formula, it may be desirable to introduce some scaling of the distance parameters that serve to define the individual segments of the winding configuration. In connection with the D-A routine that has been made available to us there is an adjustable tolerance parameter that serves to annul certain quantities (such as the output quantities $b_{n, m}$ mentioned in our earlier description) whenever these quantities are sufficiently small to be considered inconsequential. There is, moreover, a decision to be made regarding the number of derivatives that can be regarded as evaluated in a trustworthy manner from our model configuration and that accordingly merit retention in the field evaluation.

The best part of using DA is that higher derivatives can be calculated to any desired order (limited by truncation errors) and a degree of simplification introduced in the fact that a Fourier analysis is not needed and the required coil geometry is contained in its basic symmetry. The major drawback is the computation time which is dominated by the DA package and is very slow for the problem size used here ( 2 to 3 days of continuous cpu time on a SUN Sparc 1 are common). In addition we had to make several modification to the Biot-Savart formula to reduce errors. Normalizing each line current to its length (Appendix A) was proven very effective especially when long segments are present (e.g. a long straight section). We were unable so far in eliminating a difficulty that arises when a long line current connects to a much shorter one.

\section{Connection to a Fourier-Bessel Series}

It is informative to note a form that is obtained when the represention of the internal field functions $A_{n}(z)$ are replaced by simple circular functions of $\mathrm{z}$ or, more generally, by Fourier series of the form

$$
A_{n}(z)=\sum_{m=1} E_{n, m} \cos \left[(2 m-1) \frac{\pi z}{L}\right]
$$

as would be characteristic of a periodic (half-period $=\mathrm{L}$ ) alternating array of symmetric magnet elements. If we substitute this form for $A_{n}(z)$ and the corresponding forms

$$
A_{n}^{(2 k)}(z)=\sum_{m=1}(-1)^{k} E_{n, m}\left[(2 m-1) \frac{\pi}{L}\right]^{2 k} \cos \left[(2 m-1) \frac{\pi z}{L}\right]
$$

for the required derivatives of $A_{n}(z)$ into the expression proposed earlier for the scalar potential $\mathrm{V}$, we obtain (after some intermediate algebra, and recognition of the Taylor-series expansion for a modified Bessel function of the first kind)

$$
V(r, z, \theta)=\sum_{n, m} n !\left[\frac{2 L}{(2 m-1) \pi}\right]^{n} E_{n, m} I_{n}\left[\frac{(2 m-1) \pi r}{L}\right] \cos \left[(2 m-1) \frac{\pi z}{L}\right] \sin n \theta
$$


hence

$$
V(r, z, \theta)=\sum_{n} \sum_{m} G_{n, m} I_{n}\left[\frac{(2 m-1) \pi r}{L}\right] \cos \left[(2 m-1) \frac{\pi z}{L}\right] \sin n \theta
$$

where

$$
G_{n, m}=n !\left[\frac{2 L}{(2 m-l) \pi}\right]^{n} E_{n, m}
$$

which is recognized as being of such a form that $\nabla^{2} V=0$.

We note that while a harmonic component with angular dependence proportional simply to $\sin (\mathrm{n} \theta)$ contributes a dominant $\mathrm{r}$-dependence proportional to $r^{n}$, additional terms proportional to $r^{n+2}, r^{n+4}, \ldots$ arise from the series development of $I_{n}$.

Accordingly, as is also immediately evident from the development of $\mathrm{V}$ shown on p.2, "pseudo-harmonics" (as evidenced by the character of the r-dependence of such additional terms) become present in the 3-D field of pure "true harmonic order" in (and may be particularly pronounced if the field varies rapidly with respect to $\mathrm{z}$ ). Thus, as a simple example, one expects a 3-D periodic true quadrupole field $(n=2)$ also to contain pseudo-harmonic components of an octupole, dodecapole, etc. order. A true dodecapole term of course will also be present if the winding configuration is such as to introduce into the potential function terms proportional to $\sin (6 \theta)$. The terminology referring to pseudo-multipoles has been suggested by P. Krejcik in a paper presented at the 1987 IEEE Particle Accelerator Conference..$^{l}$

We demonstrate the above by replacing $A_{n}(z)$ with a pure quadrupole $(n=2)$ that is a circular function of $z$ with a truncated Fourier series $m=1$ and a half period $L=50$ :

$$
A_{2}(z)=35.346322 \cos \frac{\pi z}{50}
$$

The current density was replaced with 40 wires placed on a cylinder with of $25 \mathrm{~cm}$ radius. The location of the current segments was such that the interior field was as close as possible to a quadrupole field with a pure sinuisoidal longitudinal dependence of focusing strength. We have used 3 magnets to simulate the periodic nature in $\mathrm{z}$ and used the DA method to calculate the A's.

Washington, D. C.; March 16-19, 1987, Proc. 1987 IEEE Particle Accel. Conf., v.2, 1278-1280 


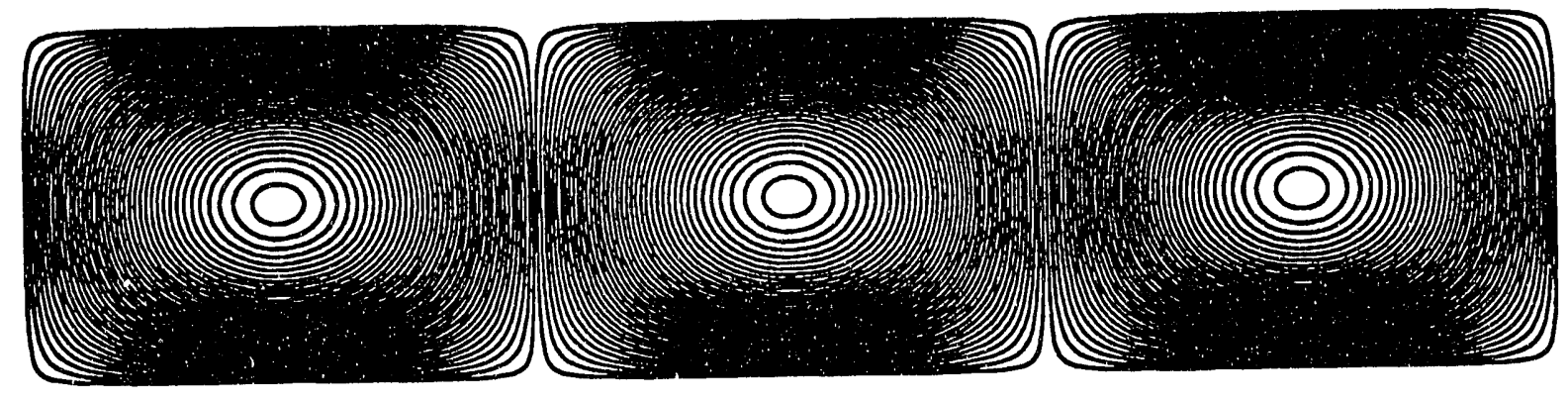

Figure 2 A truncated array of quadrupole magnets that were used to demonstrate the DA method (top view of one quadrant only for 3 half periods of the focusing field - - the lines are lines of current flow).

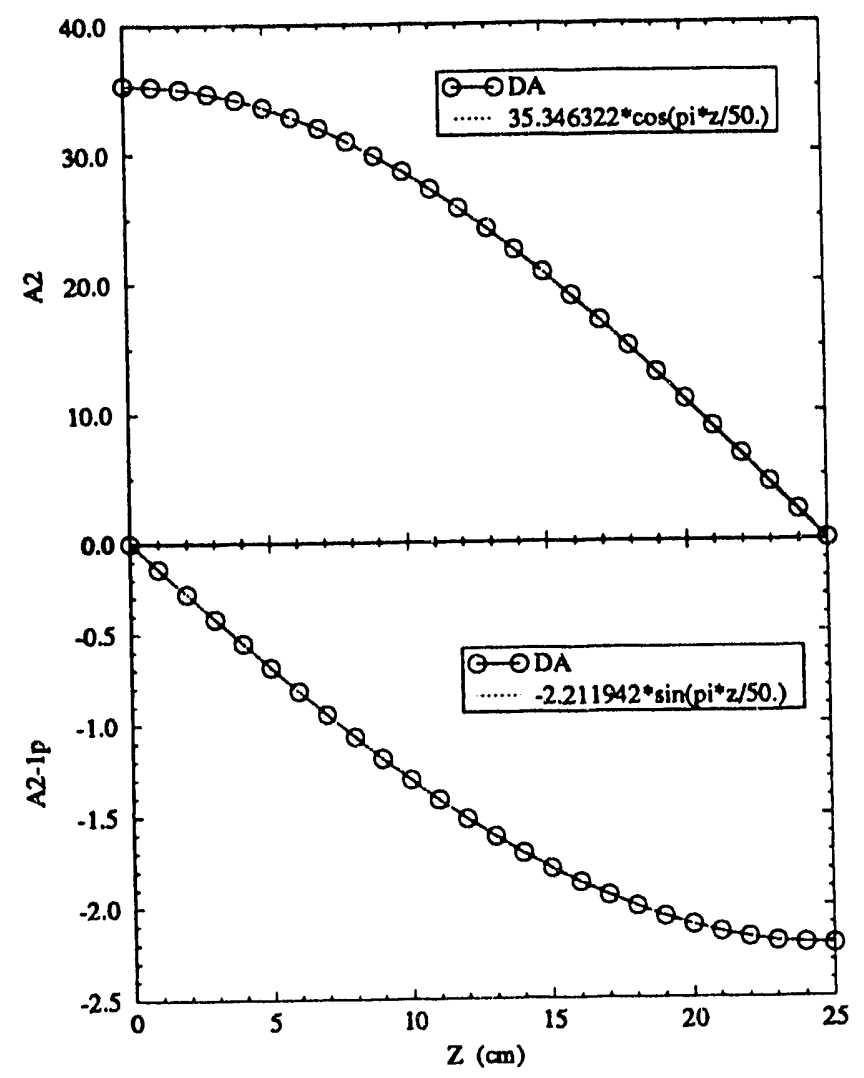

Figure 3 A2 and its first derivative showing the preservation of a simple sinusoidal variation in $\mathrm{z}$. 

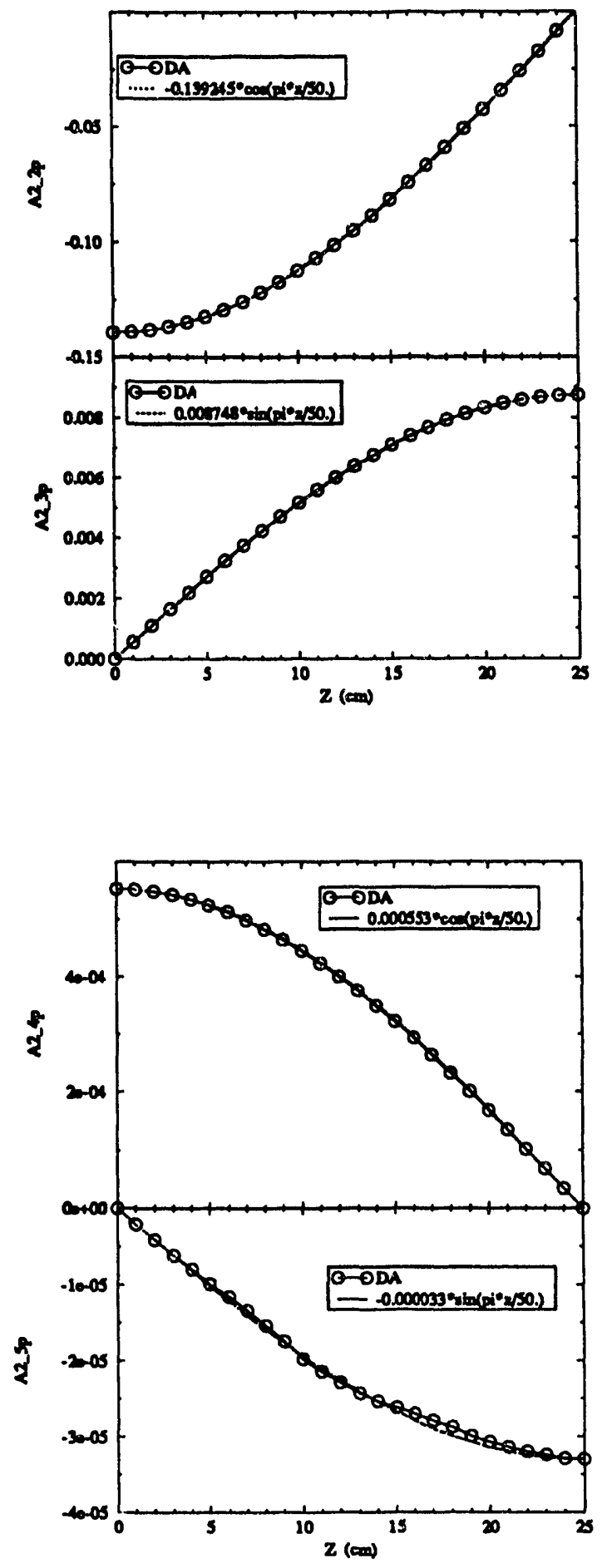

Figure 4 More derivatives of A2 showing the preservation of a simple sinusoidal variation in $\mathrm{z}$. 


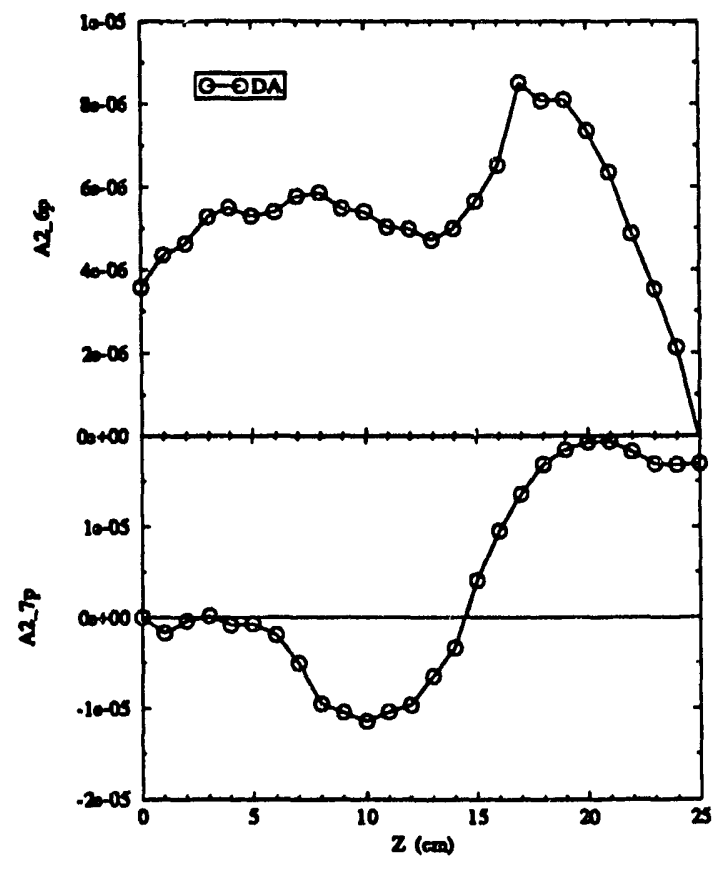

Figure 5 High derivatives showing truncation errors.

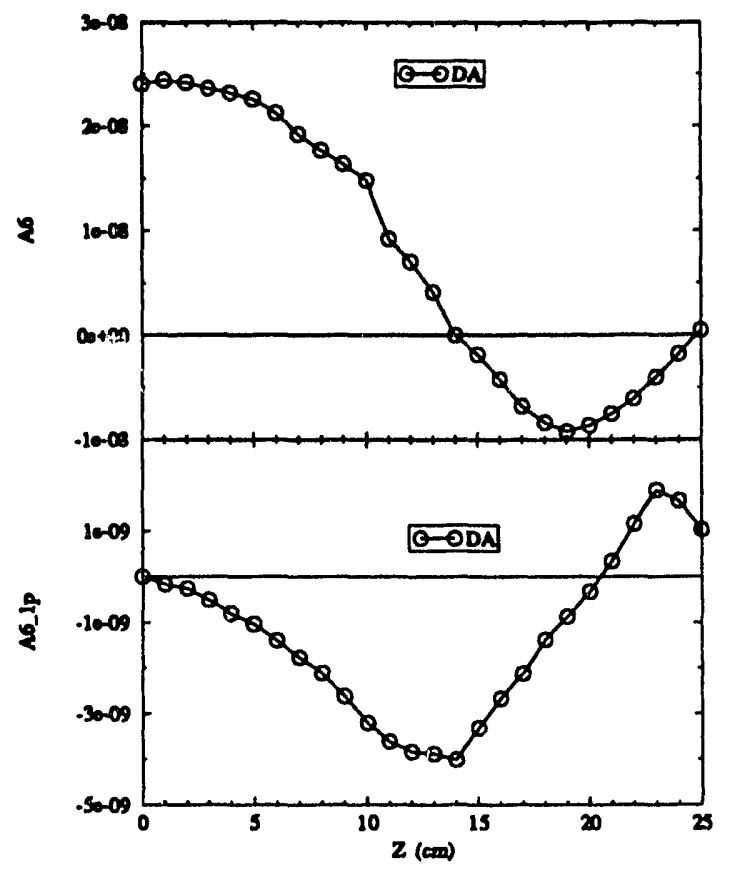

Figure 6 The use of wires destroys the purity of the quadrupole and produces a small dodecapole A6. 


\section{Single Particle Orbits}

We demonstrate the use of the A(z)'s in single-particle orbit calculations for a periodic A-G quadrupole array (as previously described) whose field gradient is a pure cosine function of the longitudinal co-ordinate $\mathrm{z}$ (see references ${ }^{\mathrm{mno}}$ ).

In Figs. 7-8 we present in some decail the increase of tune with radius from the smallamplitude value of $\sigma_{0}=72^{\circ}$ to a limiting value just before the onset of the $90^{\circ}$ resonance (at $X_{\max } / L=0.39$ ). Substantially identical results have been obtained by a Bessel-function form for the r-dependence of the focusing field and for a sequence of $A_{2}(z), A_{2}^{(k)}(z)$ with $k \leq$ 4 , and $A_{6}(z)$ functions (evaluated by linear interpolation for values of $z$ that were not integer multiples of $1 \mathrm{~cm}$ ) intended to represent the Differential-Algebra development of that field ( $\mathrm{r}=25 \mathrm{~cm}$ and half period $\mathrm{L}=50 \mathrm{~cm}$ ). A pair of phase plots (Fig. 9), obtained by these two methods and depicting $\frac{d X}{d Z}$ vs. $X$ at successive centers of $X$-focusing quadrupoles, shows the unstable order-4 fixed points $\left(\sigma=90^{\circ}\right)$ with an apparently closed-curve stability boundary lying just inside the region at $|X|=19.5 \mathrm{~cm}$. Also shown on the phase plots are the locally stable $90^{\circ}$ fixed points ( surrounded by small, presumably locally stable, closed curves situated in a more generally unstable region), some points at $|X| \approx 15.58$ etc \& at $\left|\frac{d X}{d Z}\right| \approx 0.101$ for $\sigma=80^{\circ}$, and an additional closed curve launched with $\mathrm{X}_{0}=14.0 \mathrm{~cm}\left(\sigma\right.$ between $78^{\circ}$ and $\left.79^{\circ}\right)$.

$m$ Single Particle Orbits in the Magnetic Field of the ILSE Current-Dominated Quadrupole Victor Brady, HIFAR NOTE-188, February 25, 1988.

n See, as an introductory note to HIFAR-427, a Single-Particle Aperature-Limit graph for particles moving with one transverse spatial degree of freedom in an A-G (electric or magnetic) quadrupole array with a gradient that has a purely sinusoidal variation with respect to the longitudinal co-ordinate $z$. It is noteworthy that on this graph one finds aperature ratios that with magnetic quadrupole focusing may be virtually twice those obtainable by use of an electric quadrupole lattice.

- HIFAR Note-345 and 346. 


\begin{tabular}{|l|l|l|}
\hline $\begin{array}{l}\mathrm{X}_{\mathbf{0}} \\
(\mathrm{cm})\end{array}$ & $\begin{array}{l}\sigma \text { (Bessel Function) } \\
(\text { deg })\end{array}$ & $\begin{array}{l}\sigma \text { (D-A) } \\
\text { using field from 2 adjacent } \\
\text { quads. (deg) }\end{array}$ \\
\hline 1. & 72.04 & 72.04 \\
\hline 5. & 72.80 & 72.80 \\
\hline 10. & 75.24 & 75.24 \\
\hline 14. & - & - \\
\hline 15.57 or 15.59 & 80.00 & 80.00 \\
\hline 19.0 & 84.80 & 84.69 \\
\hline 19.5 & 86.37 & 86.11 \\
\hline 19.55 & 90.00 & - \\
\hline 19.62 & - & 90.00 \\
\hline
\end{tabular}

Figure 7 The change of wne $\left(\sigma_{0}=72^{\circ}\right)$ using Bessel functions and A's to represent the quarupole field. 


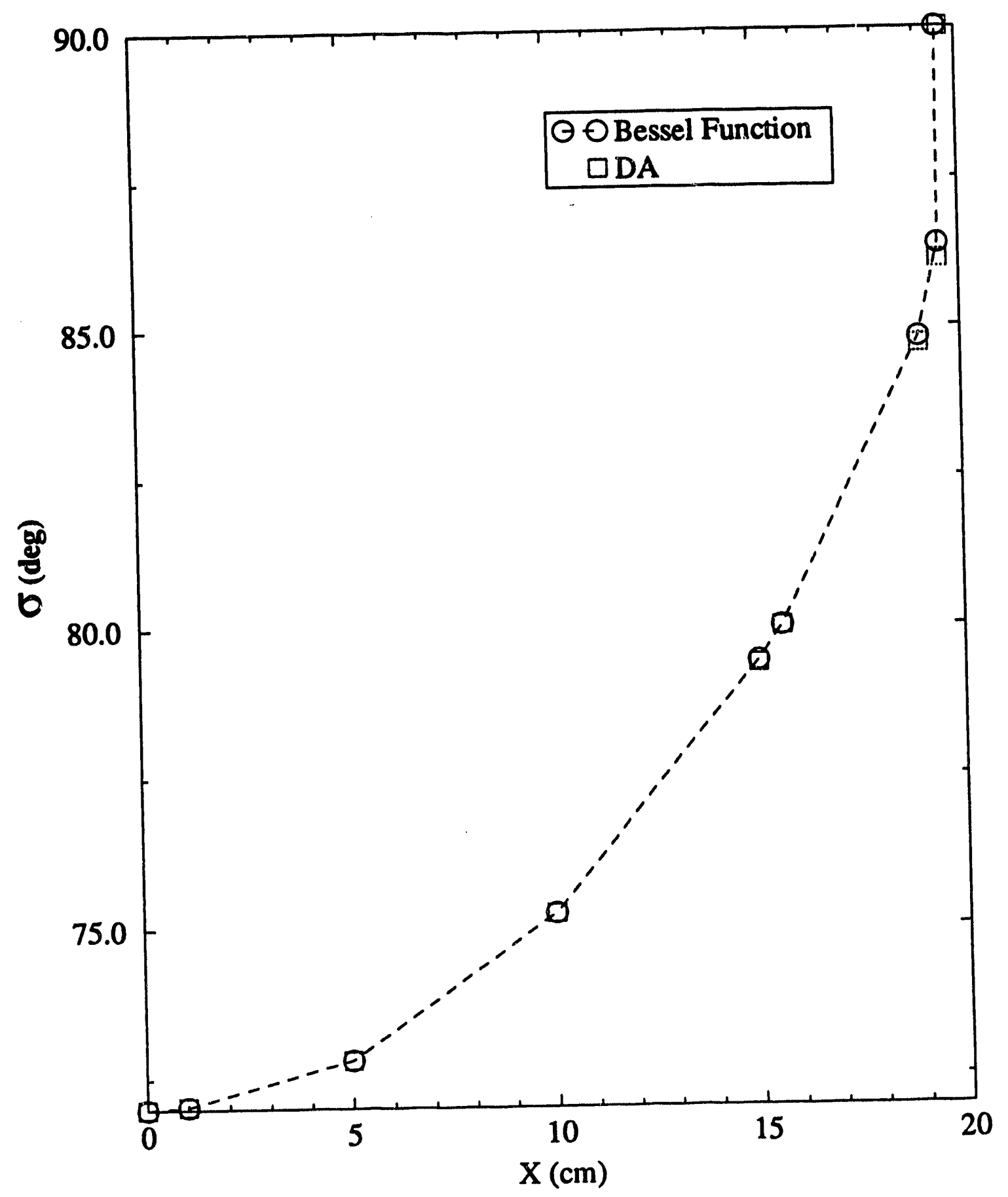

Figure 8 Single-particale TUNE for median-plane motion using Bessel-Function field $\vec{J} \propto \cos \left(\frac{\pi z}{i}\right) \cos 2 \phi \hat{e}_{z}+\frac{\pi a}{2 i} \sin \left(\frac{\pi z}{1}\right) \sin 2 \phi \hat{e_{\phi}}$ at $r=a=25$ and DA reconstructed field from $A_{2}(z), A_{2}^{k}(z)$ with $k \leq 4$, and $A_{6}(z)$. 


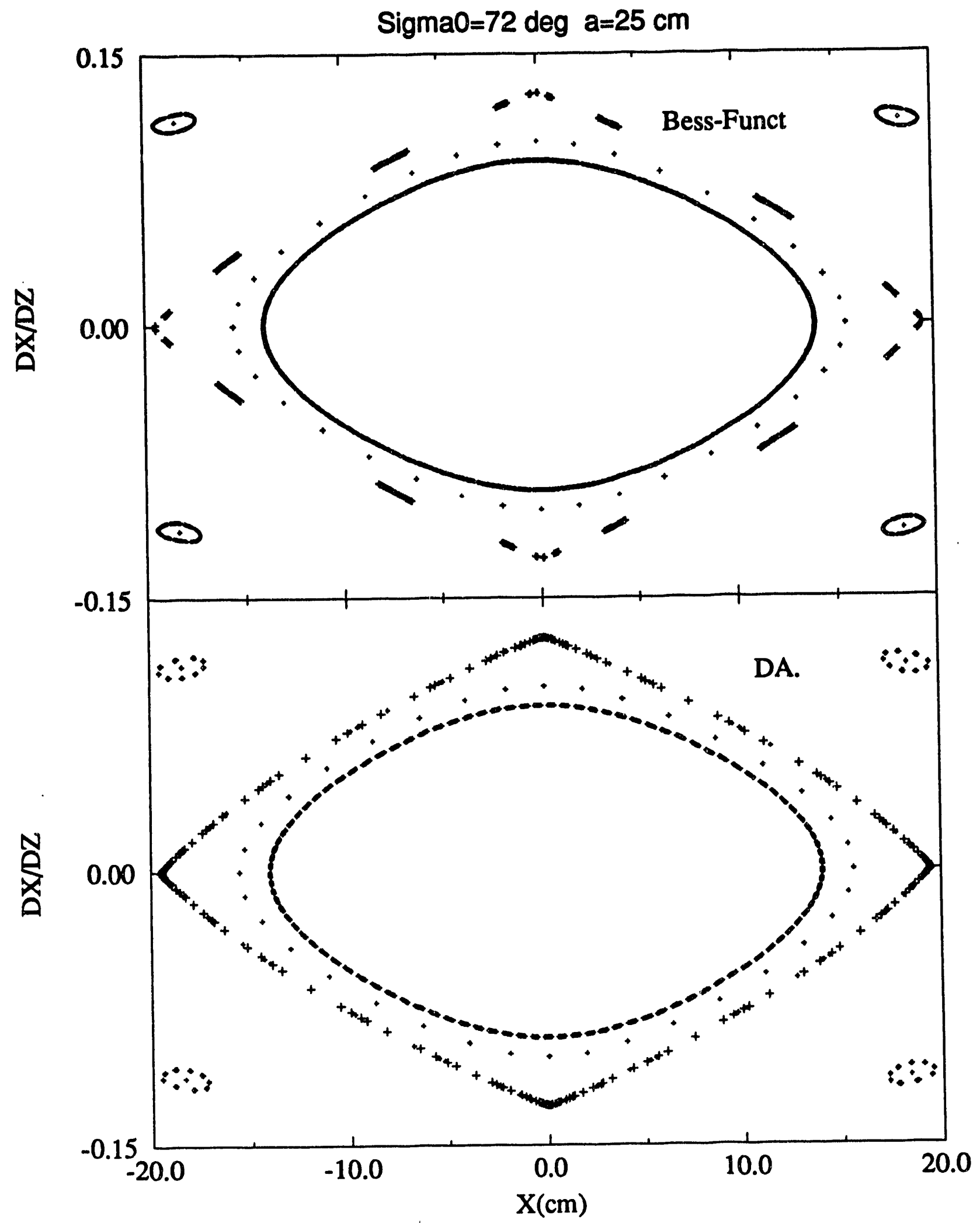

Figure 9 A pair of phase plots, obtained by the previously described methods. 


\section{Part 2- Magnetic fleid in the SSC Quad end.}

The overall "end" configuration of the quad is shown in the first five of the following figures. We have computed the $A(z)$ functions based on the numerical, DA, and Biot-Savart methods and compared the results at different radii - $r=0.5,1.0,1.5,1.7$, and $1.9 \mathrm{~cm}$ or at $25 \%, 50 \%$, $75 \%, 85 \%$, and $95 \%$ of the $2 \mathrm{~cm}$ bore radius. For the transverse field the best and worst result give an error of 1 part in $10^{5}$ at $25 \%$ and 2 parts in 100 at $95 \%$. Correspondingly the errors in $B_{2}$ are 5 parts in $10^{5}$ at $25 \%$ and 15 parts in 100 at $95 \%$ ( Fig. 15). The configuration of the end of the dipole windings is likewise shown in Figs. 20-22.
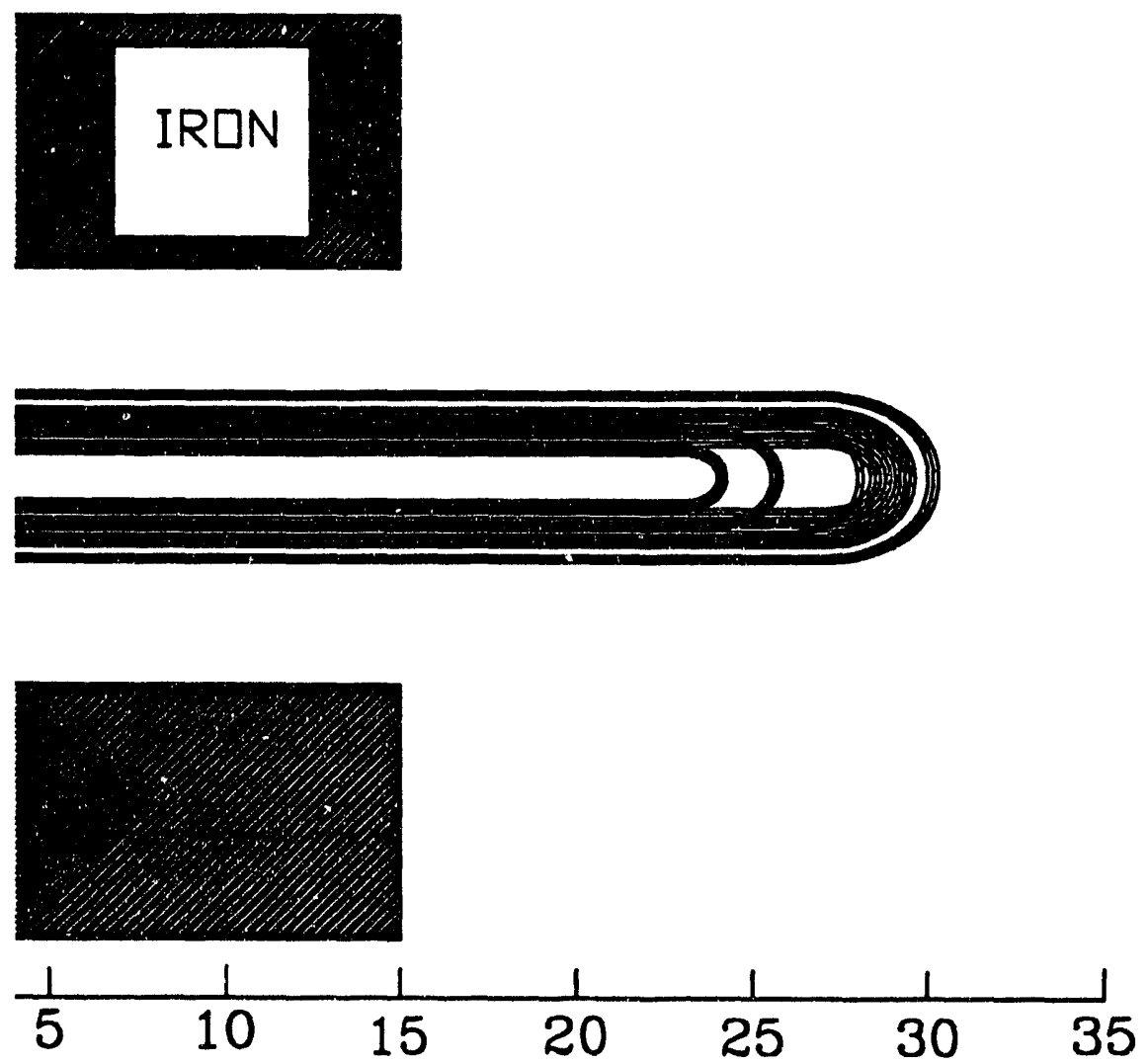

Figure 10 Coil and IRON in the end region of the SSC quad. 


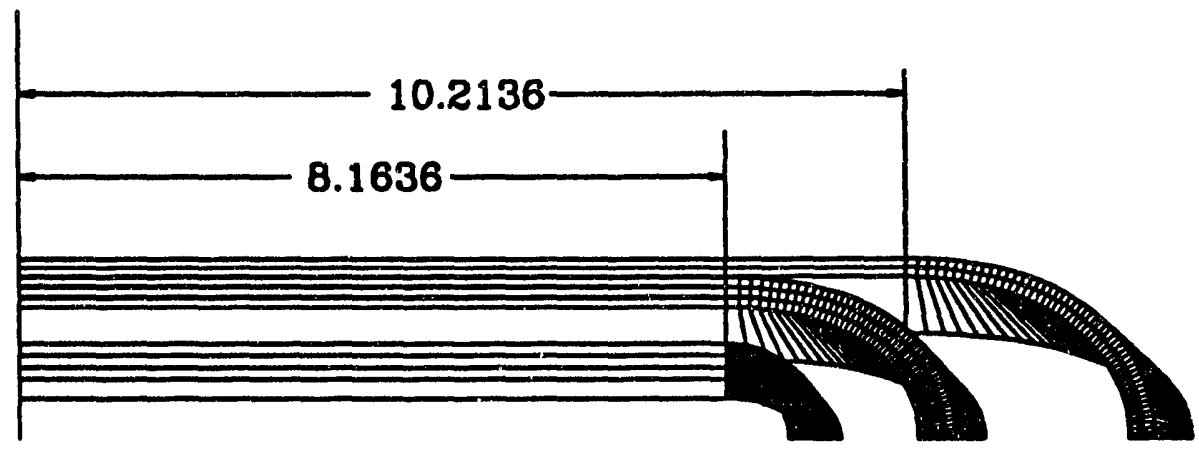

Figure 11 Conductor geometry in the end region of the SSC quad - LAYER-1 TOP.

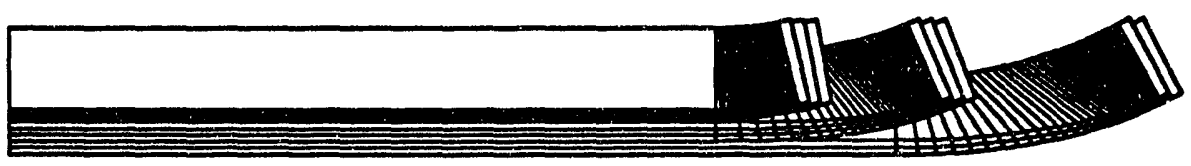

Figure 12 Conductor geometry in the end region of the SSC quad - LAYER-1 SIDE.

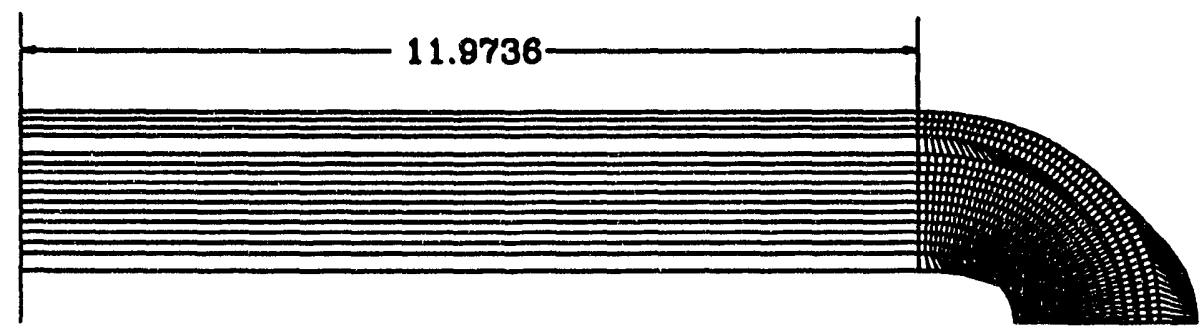

Figure 13 Conductor geornetry in the end region of the SSC quad - LAYER-2 TOP.

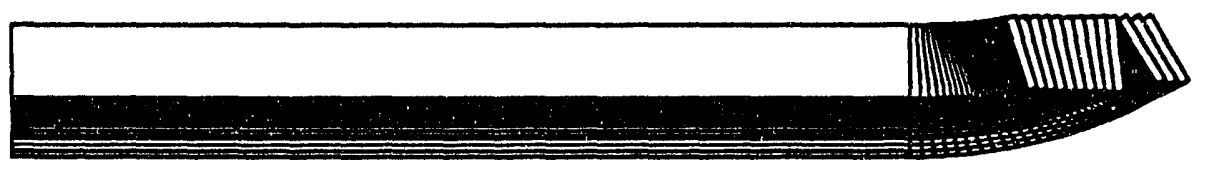

Figure 14 Conductor geometry in the end region of the SSC quad - LAYER-2 SIDE. 


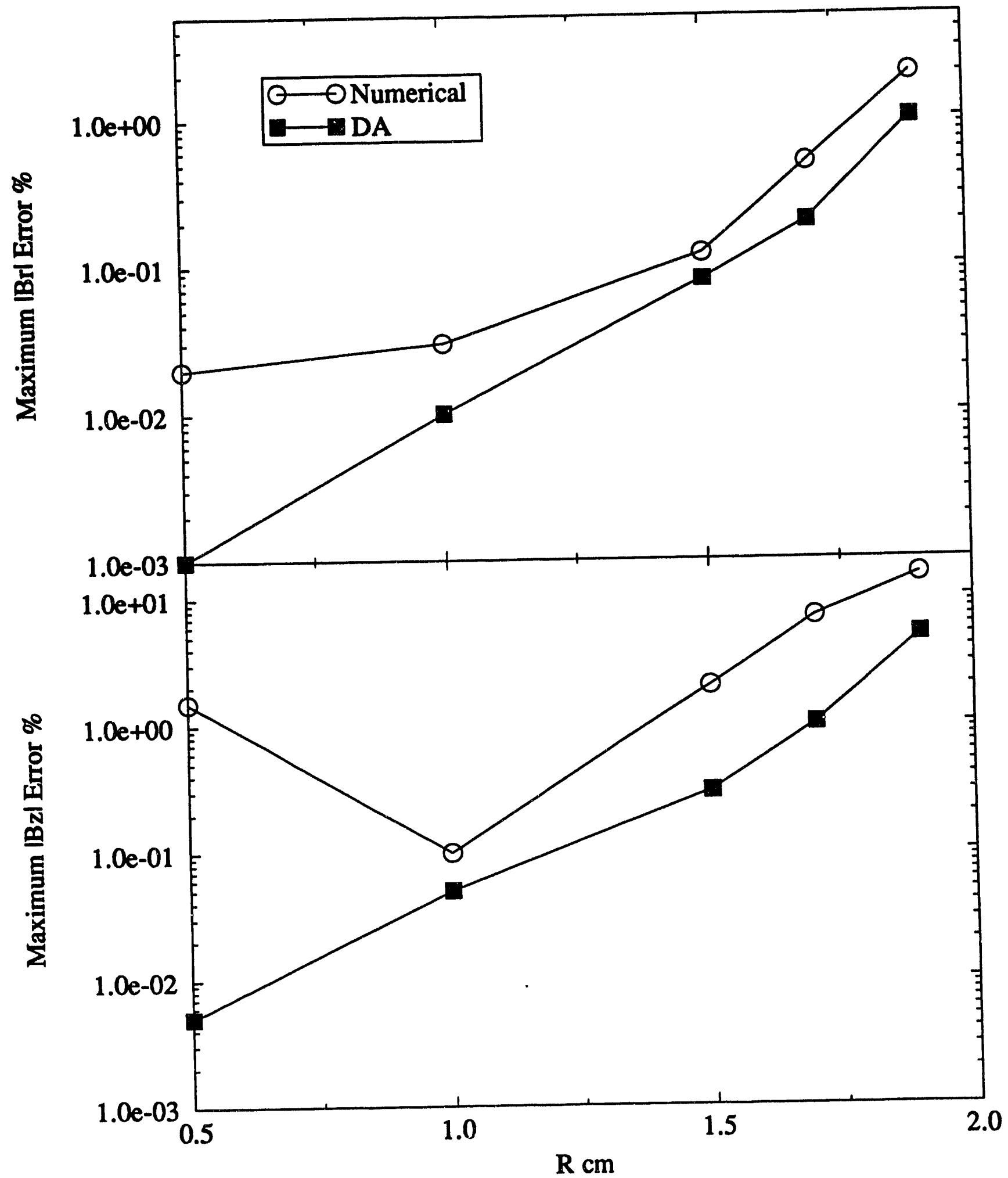

Figure 15 Maximum error associated with reconstruction of field components from the As (the windings are located at $R=2.0 \mathrm{~cm}$ ). 

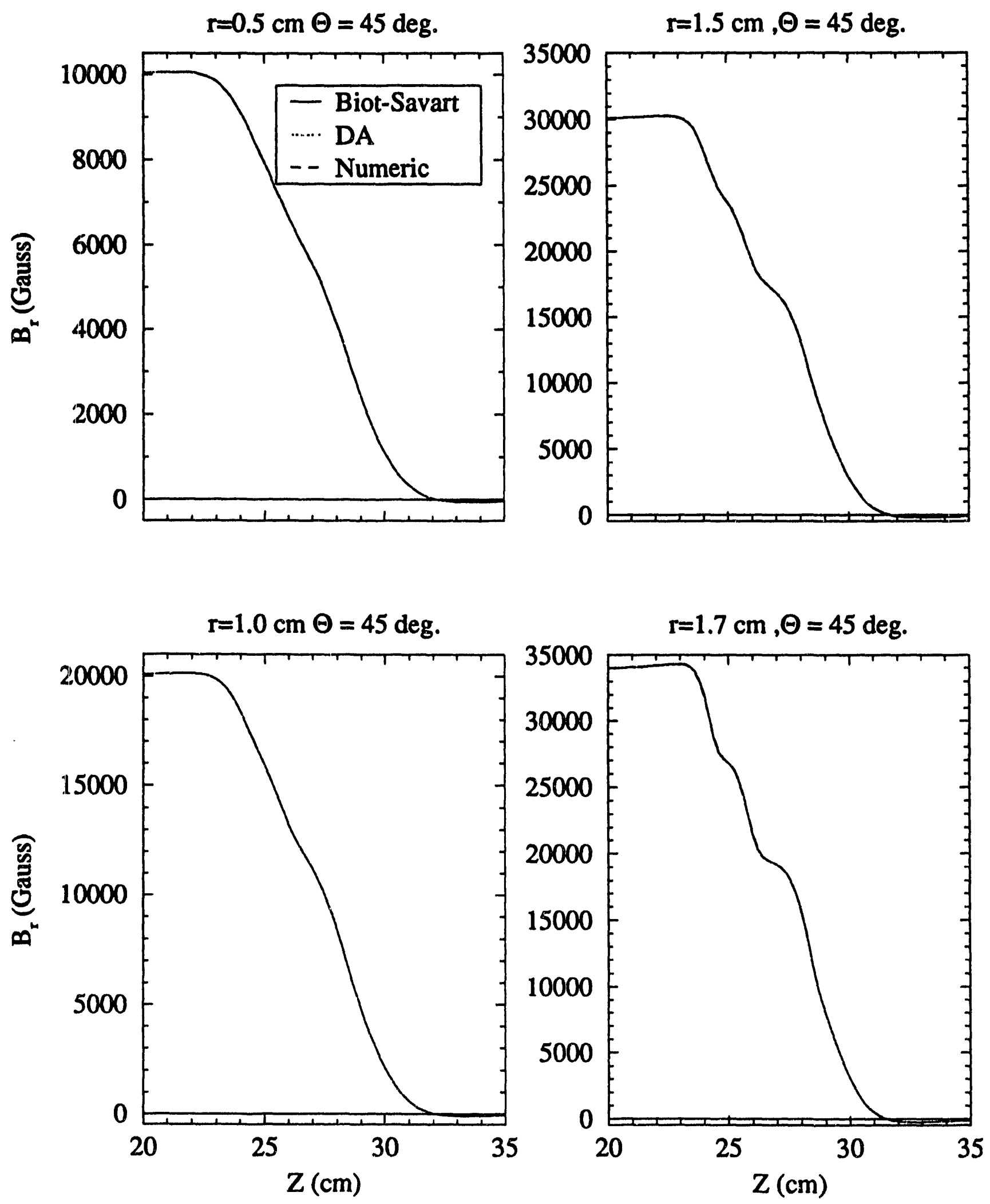

Figure 16 Transverse magnetic field at $\theta=45$ and different radii computed at the "end" region of the SSC QUAD. Results are compared between Bio_Savart and those reconstructed from the As. 


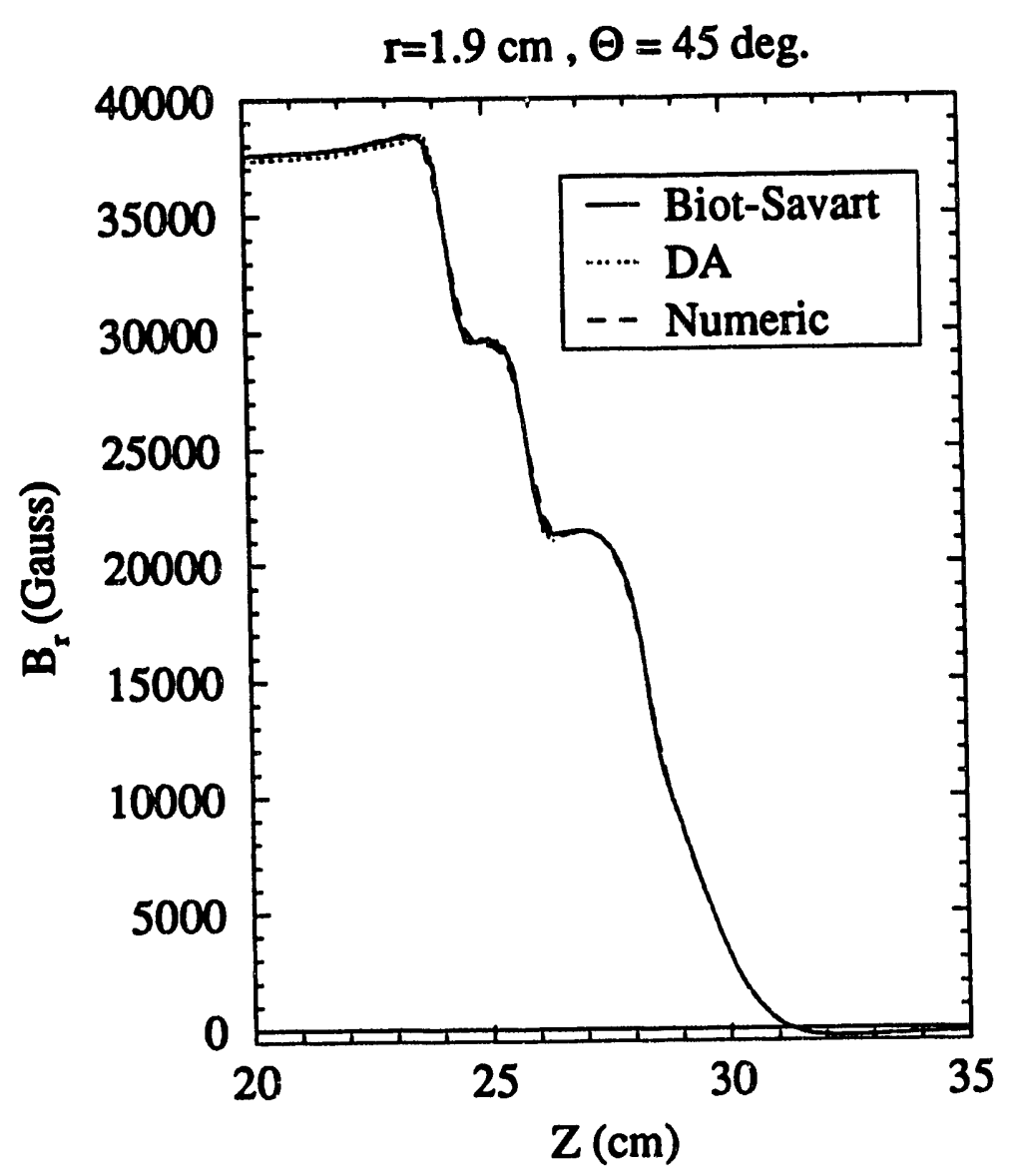



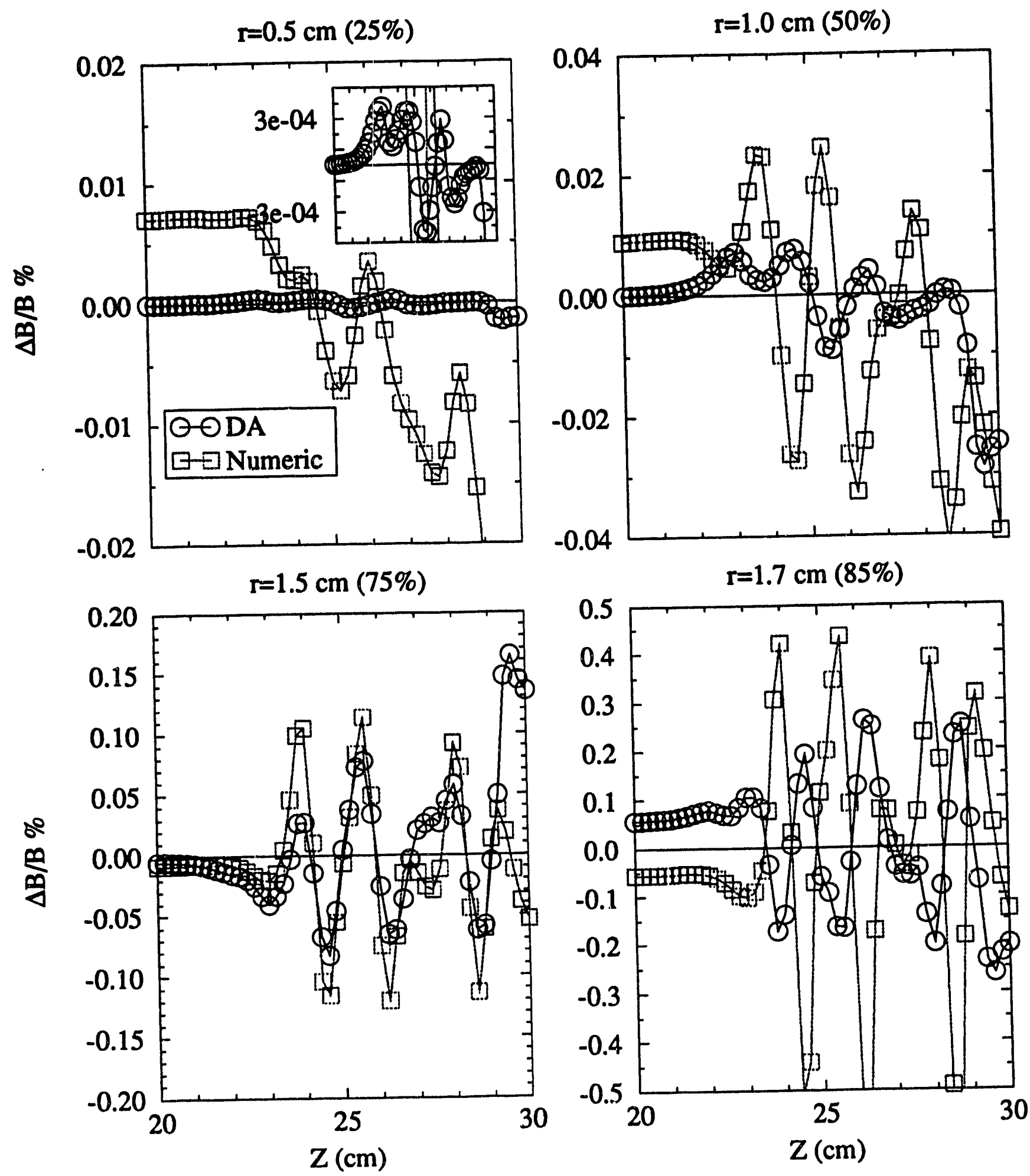

Figure 17 Error associated with the transverse magnetic field. 


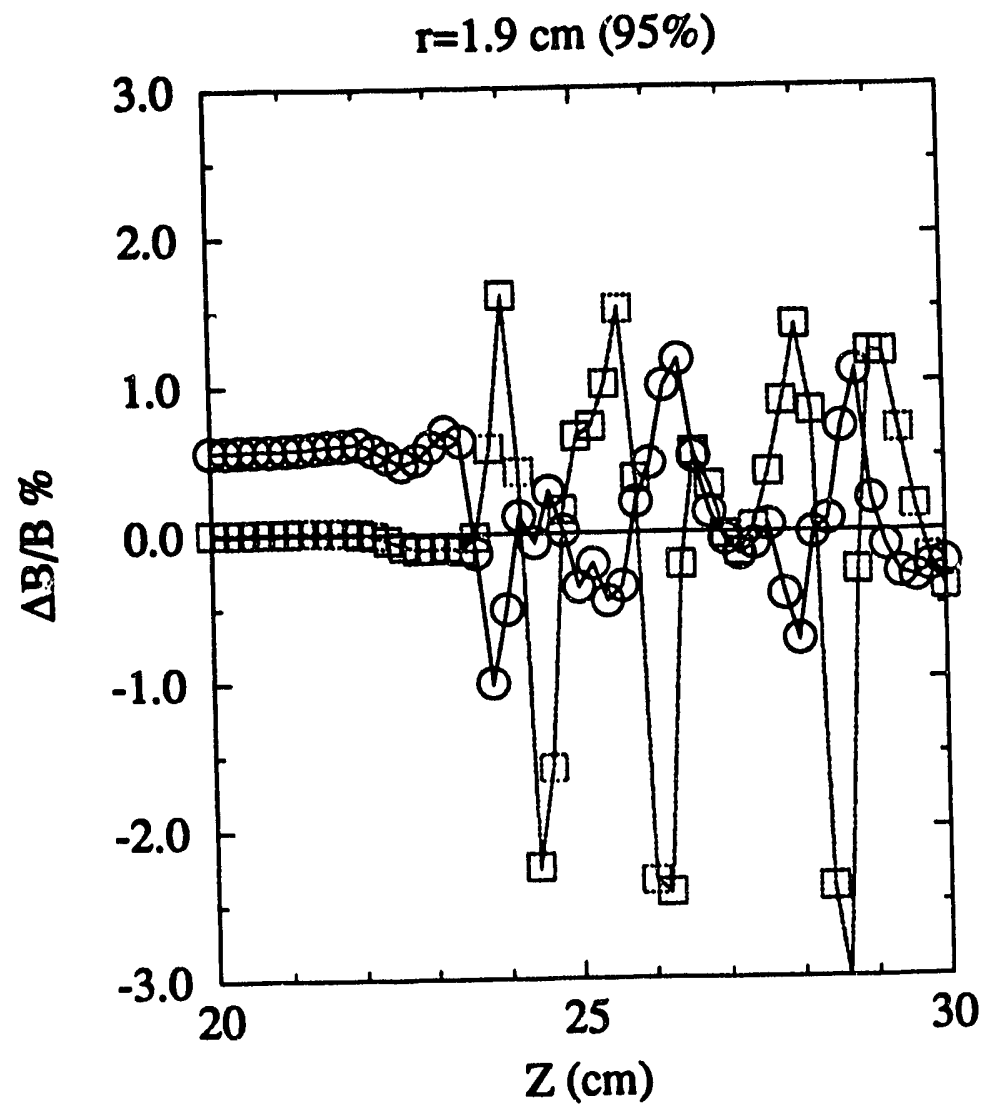



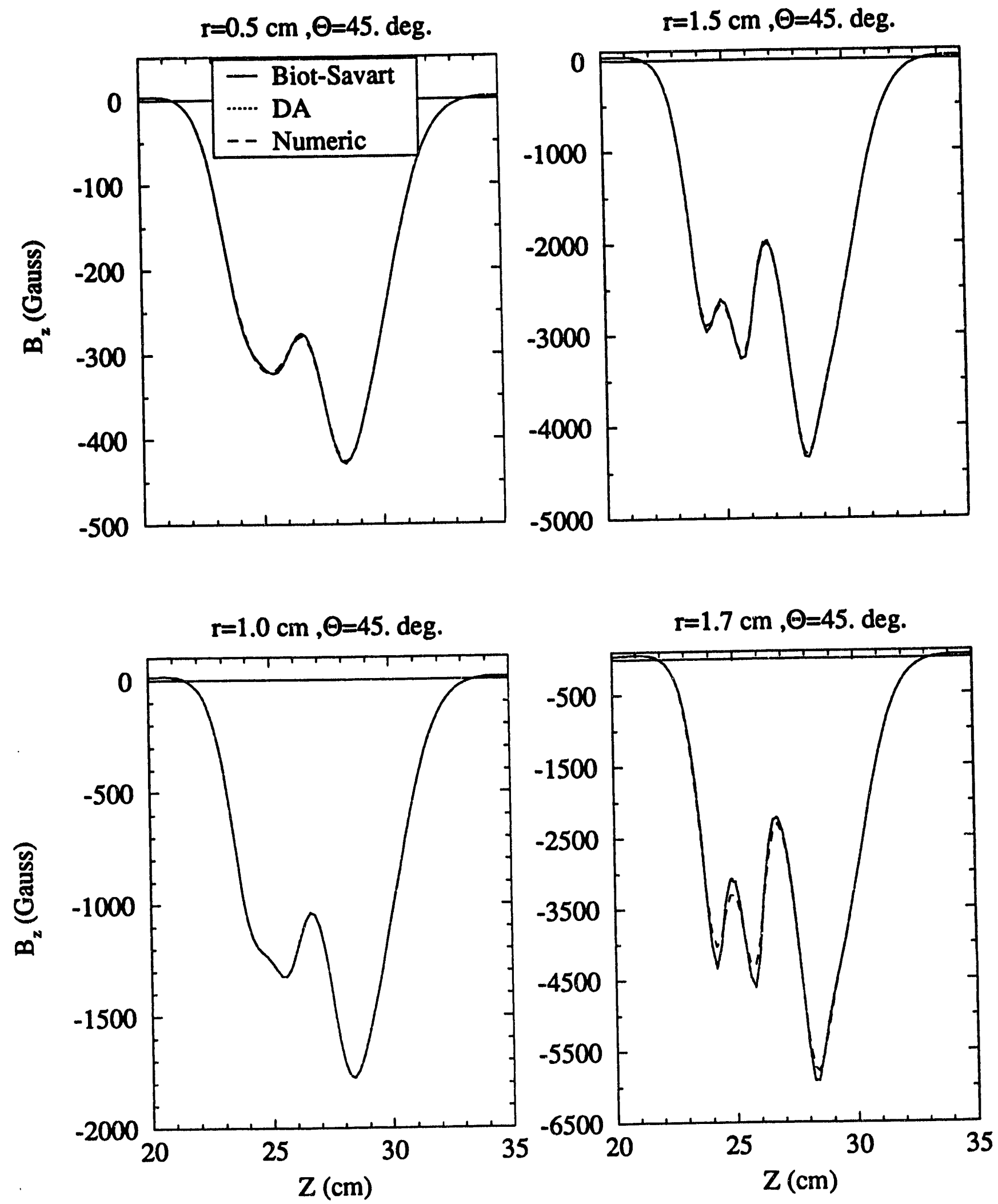

Figure 18 The magnetic field $\mathrm{Bz}$ at $\theta=45$ and different radii computed at the "end" region of the SSC QUAD. Results are compared between Bio_Savart and those reconstructed from the As. 


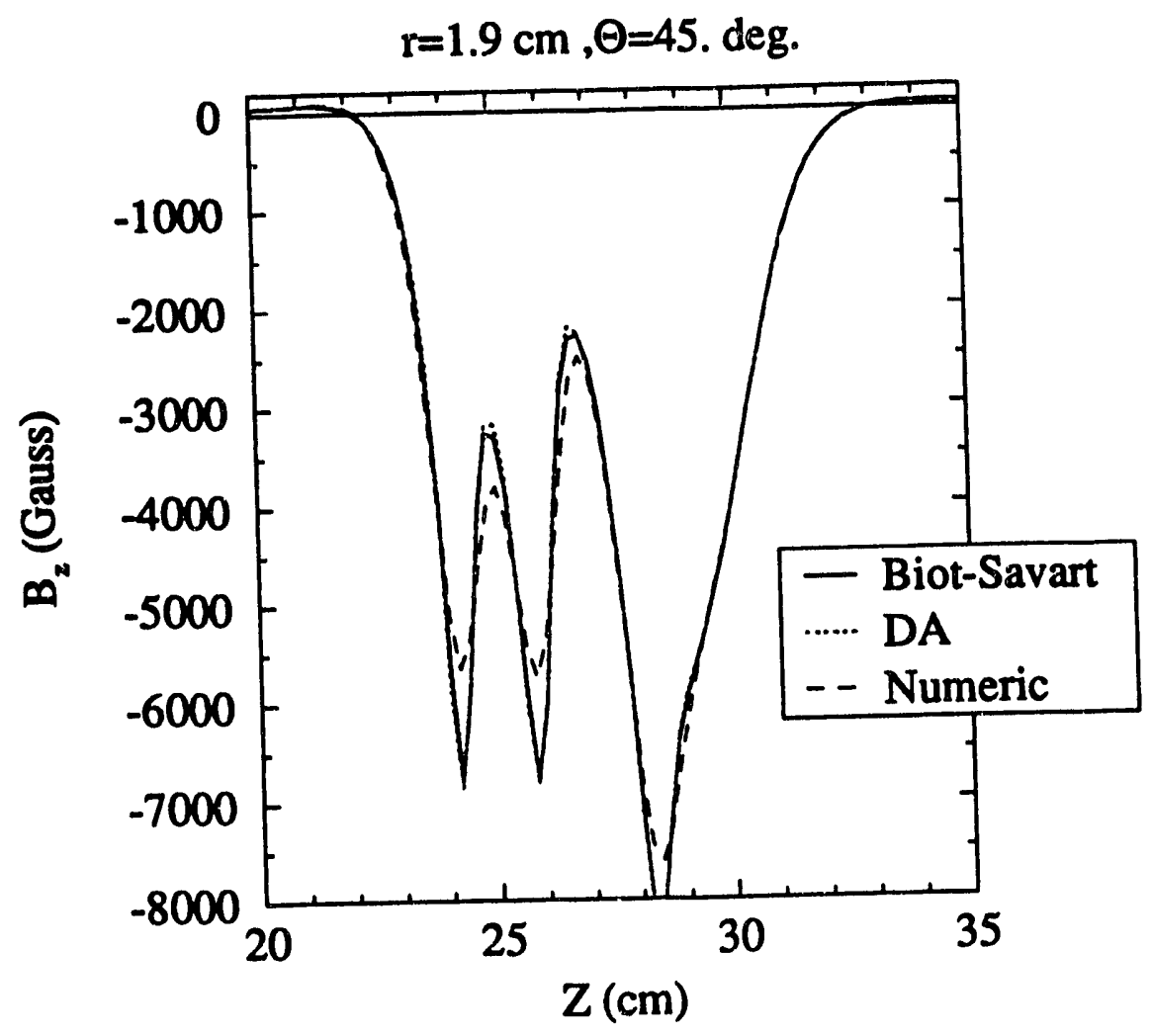



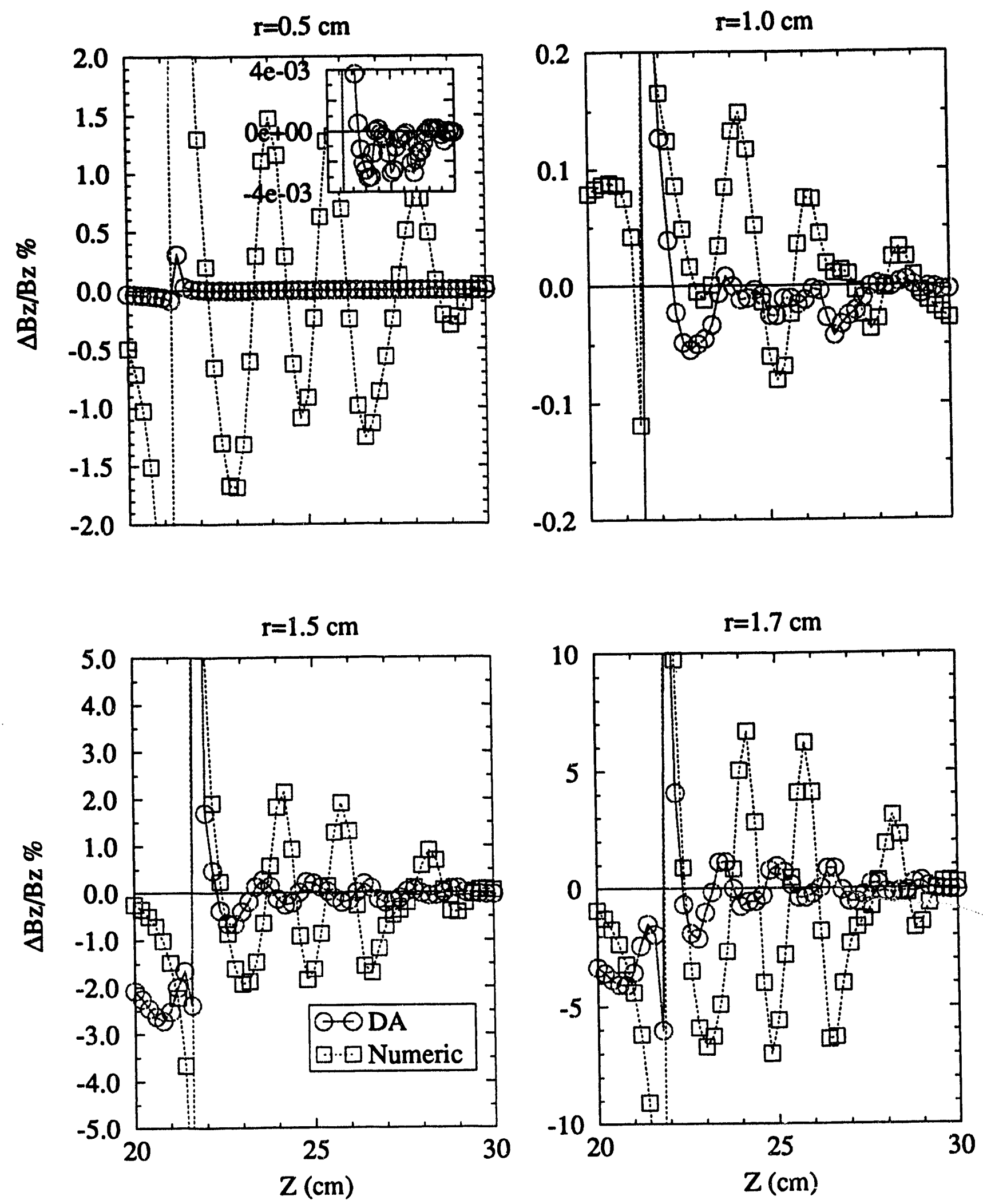

Figure 19 Error associated with the axial magnetic field Bz. 

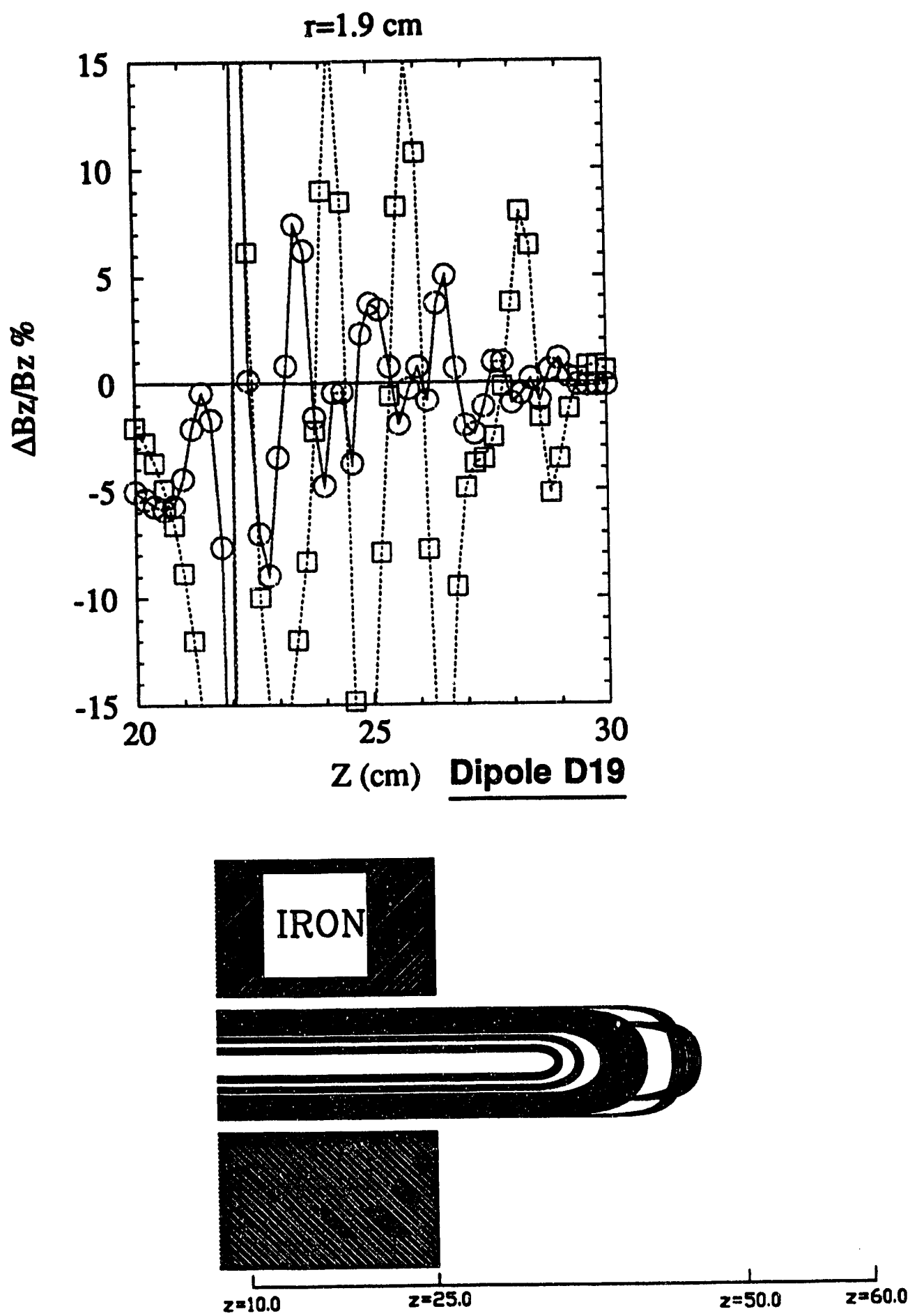

Figure 20 Coil and IRON in the end region of DIPOLE D19. 

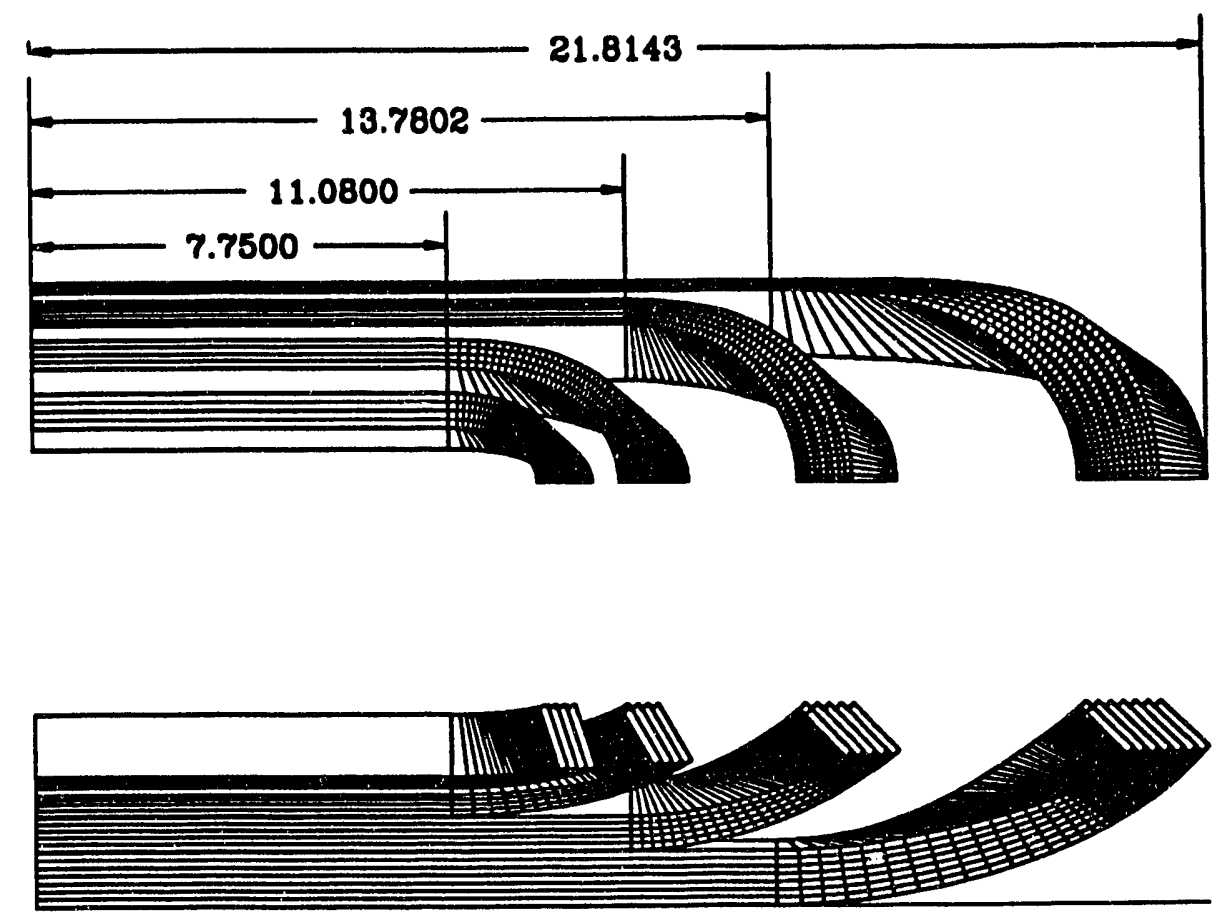

Figure 21 Conductor geometry in the end region of Dipole D19 - LAYER-1, TOP and SIDE view.
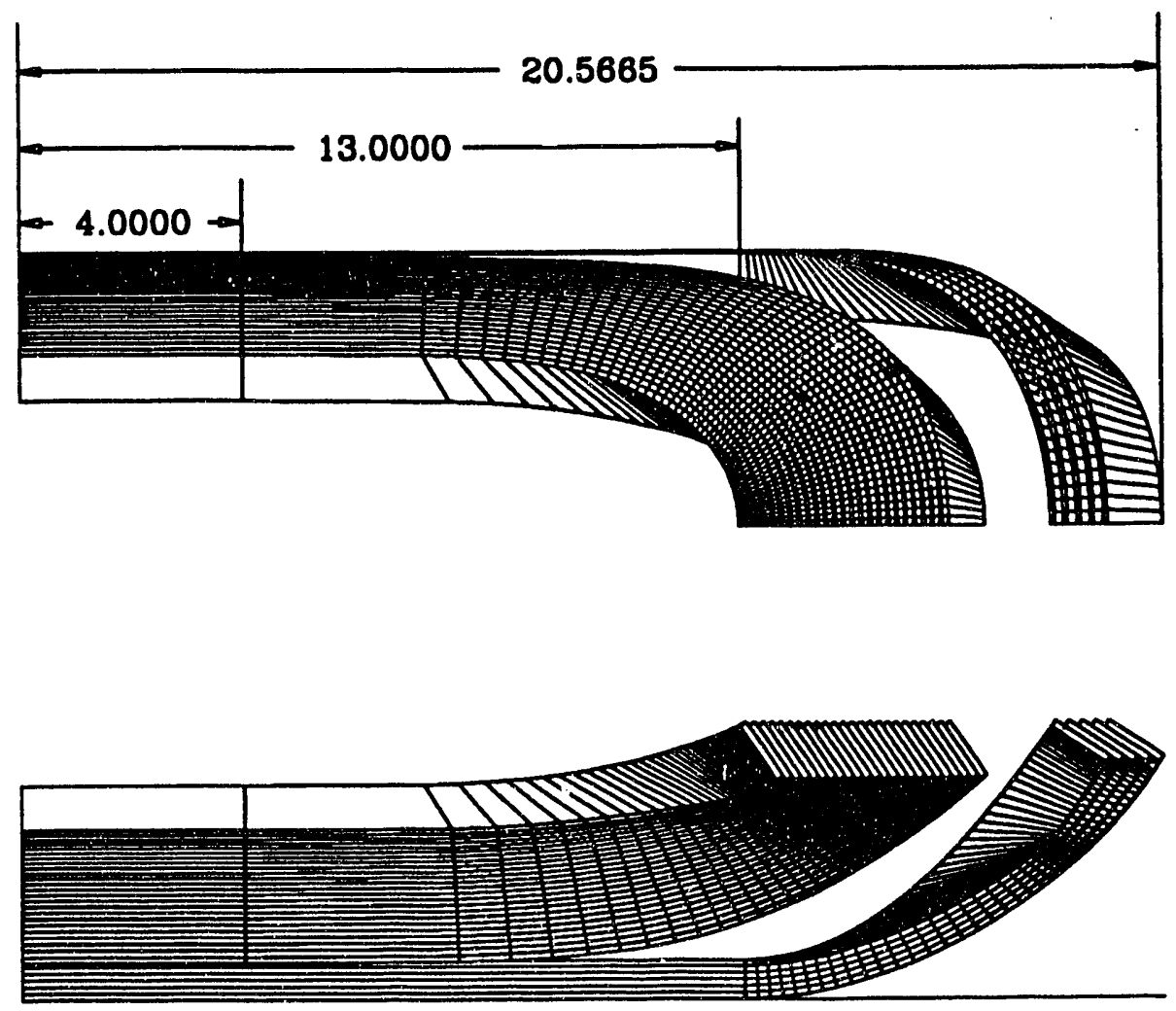

Figure 22 Conductor geometry in the end region of Dipole D19 - LAYER-2, TOP and SIDE view. 


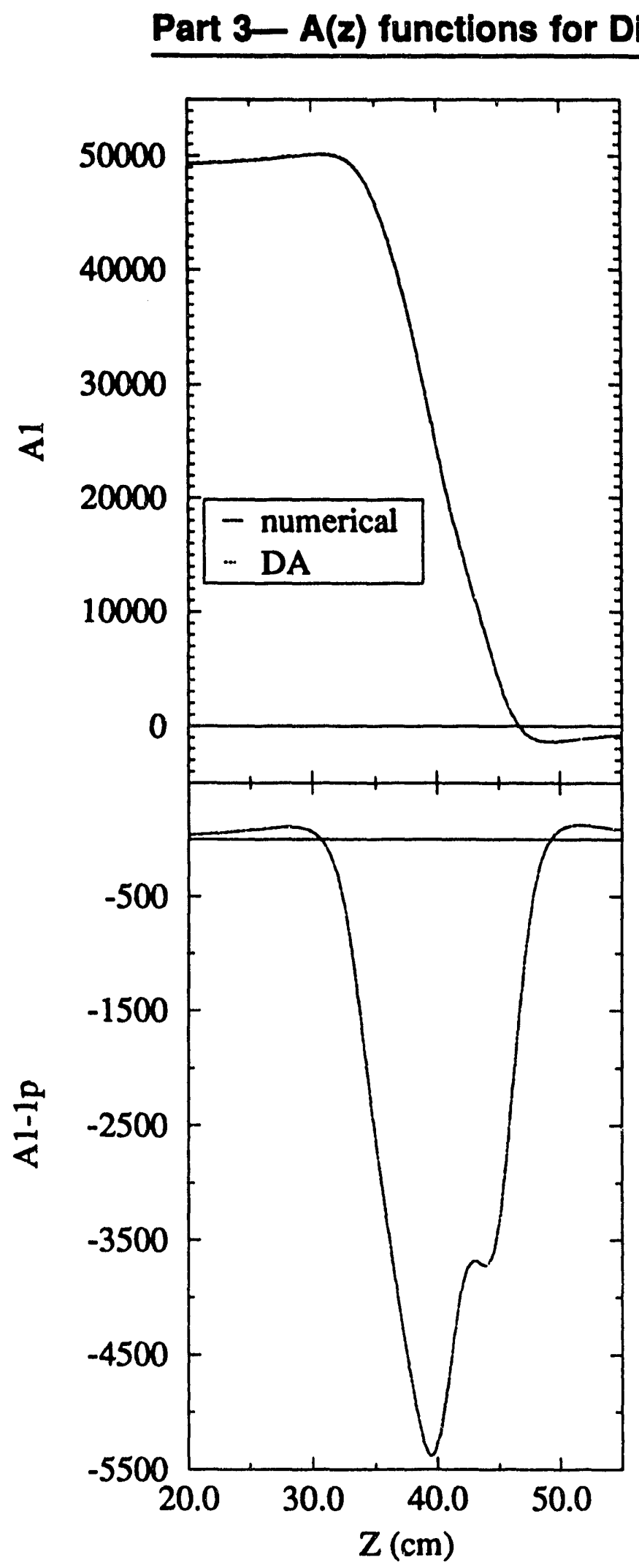

Dipole D19, and SSC Quad QC.

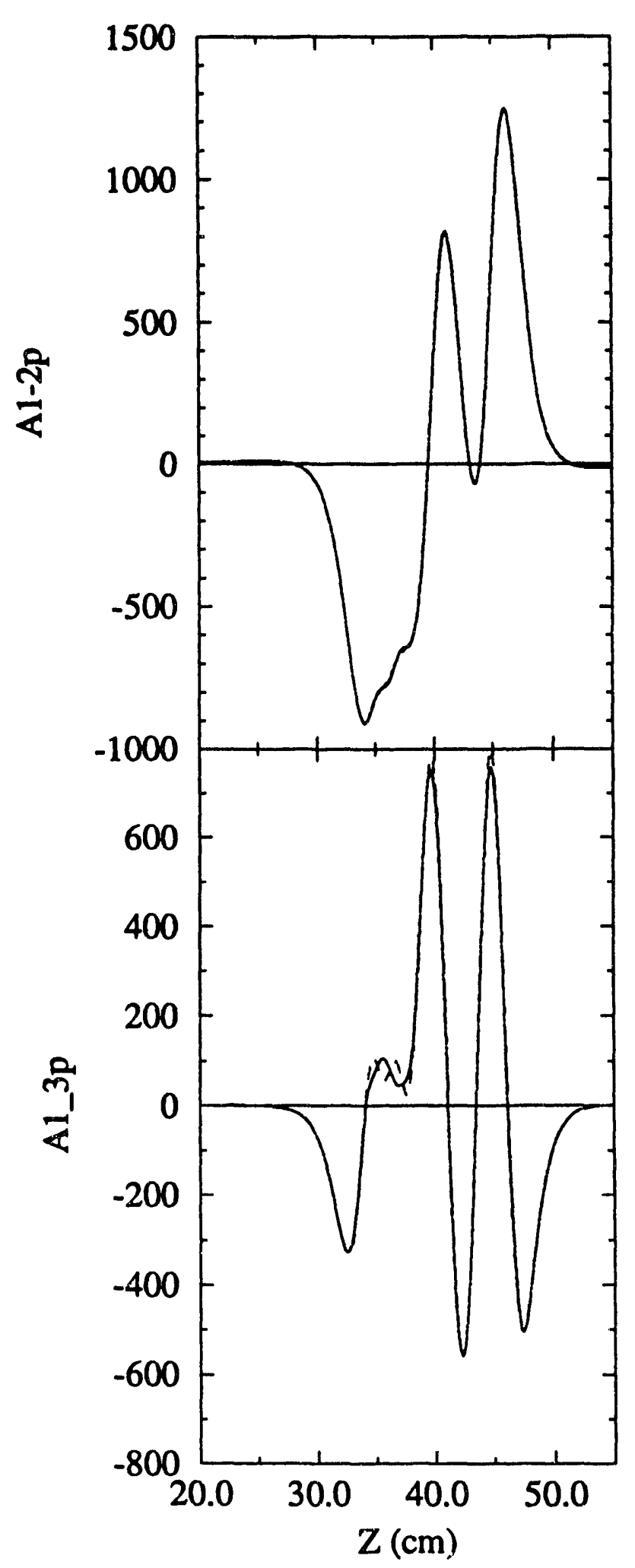

Figure 23 The DIPOLE function ( $\mathrm{n}=1$ ) $\mathrm{Al}(\mathrm{z})$ and pseudo harmonics $\frac{\partial A_{1}(z)}{\partial z}, \frac{\partial^{2} A_{1}(z)}{\partial z^{2}}, \frac{\partial^{3} A_{1}(z)}{\partial z^{3}}$. 

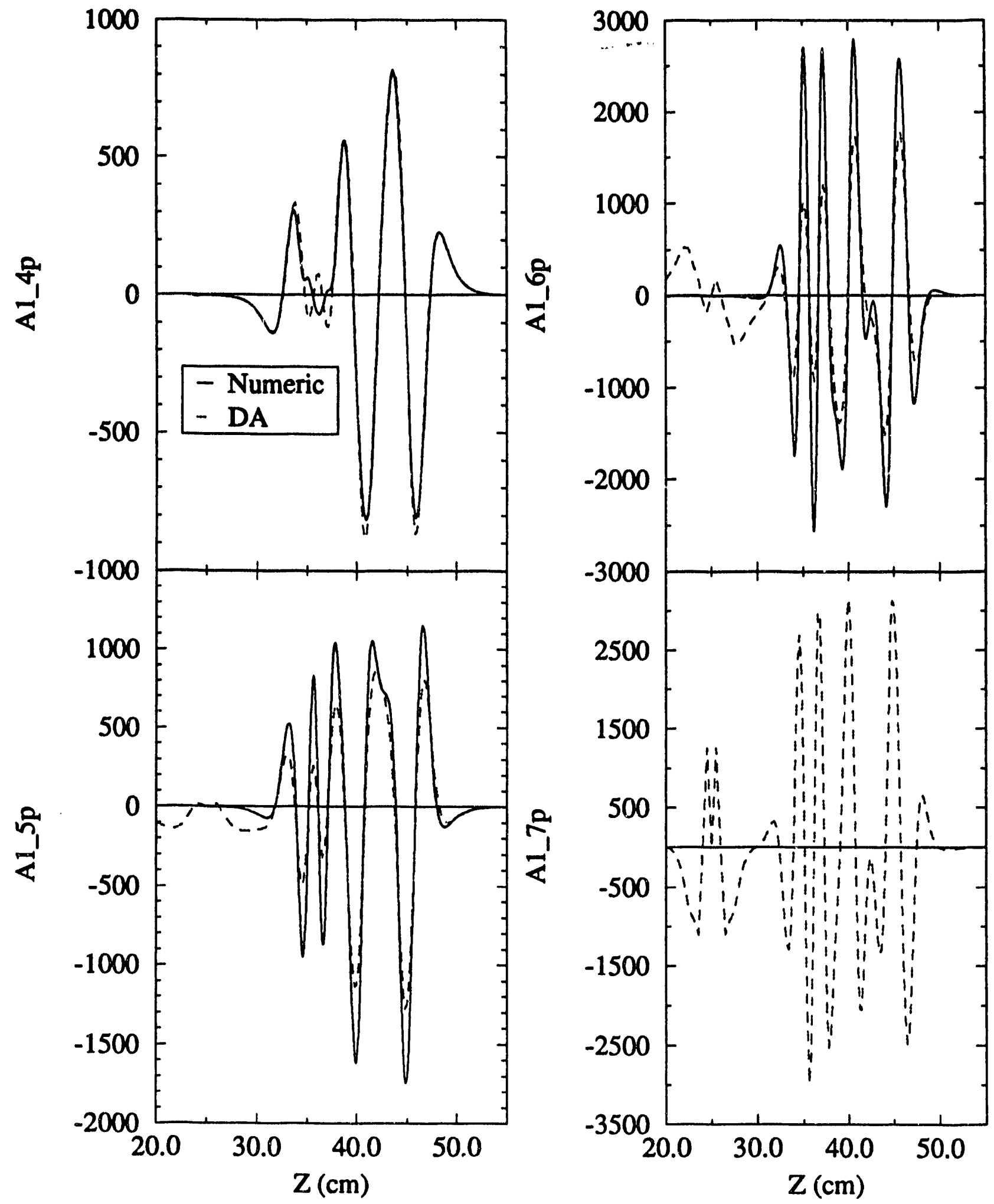

Figure 24 Pseudo harmonics of $\mathrm{Al}(\mathrm{z}) \frac{\partial^{4} A_{1}(z)}{\partial z^{4}}, \frac{\partial^{8} A_{1}(z)}{\partial z^{8}}, \frac{\partial^{8} A_{1}(z)}{\partial z^{6}}, \frac{\partial^{7} A_{2}(z)}{\partial z^{7}}$. 


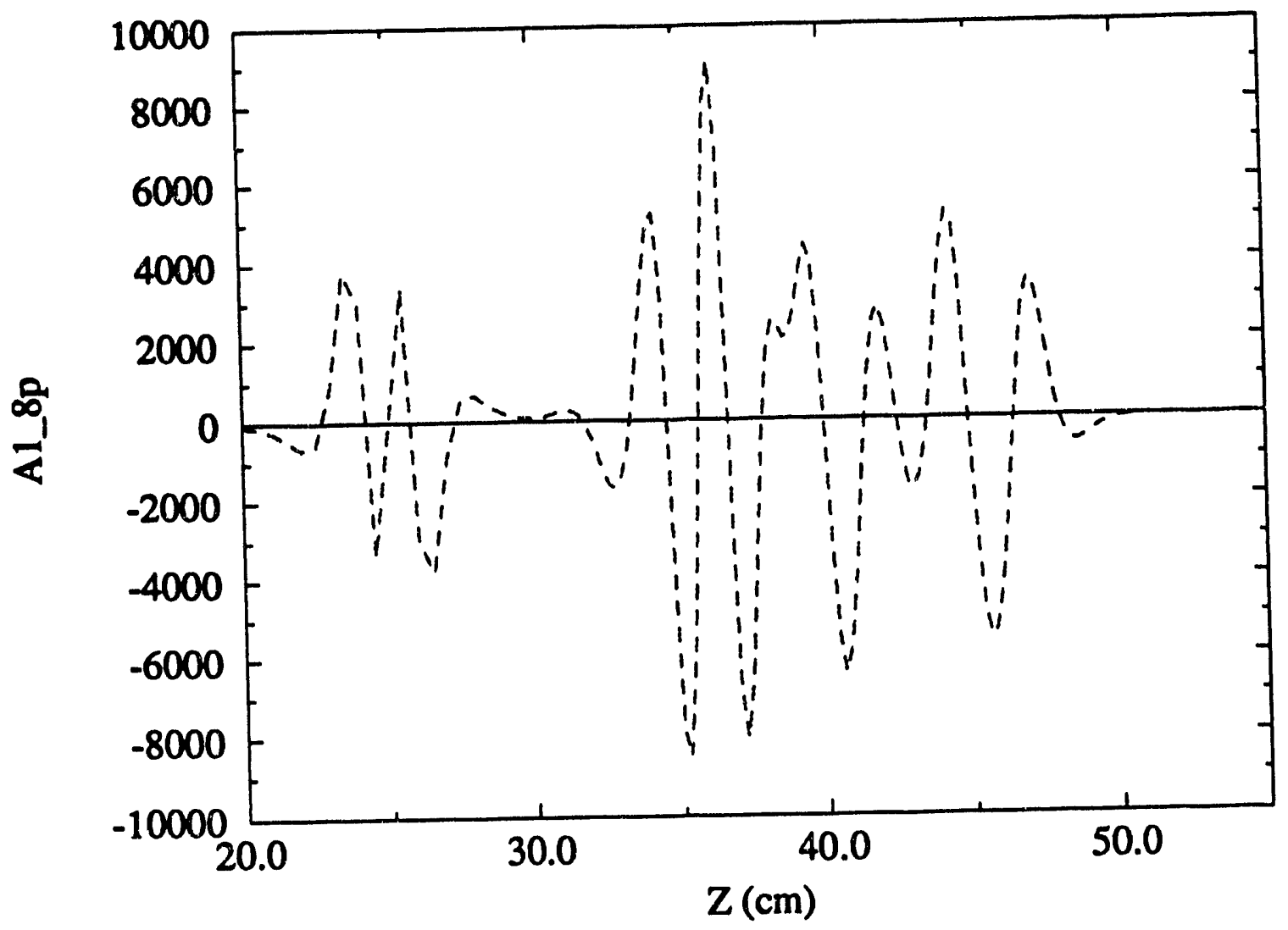

Figure 25 Pseudo harmonics of $A 1(z) \frac{\partial^{0} A_{1}(z)}{\partial z^{2}}$. 

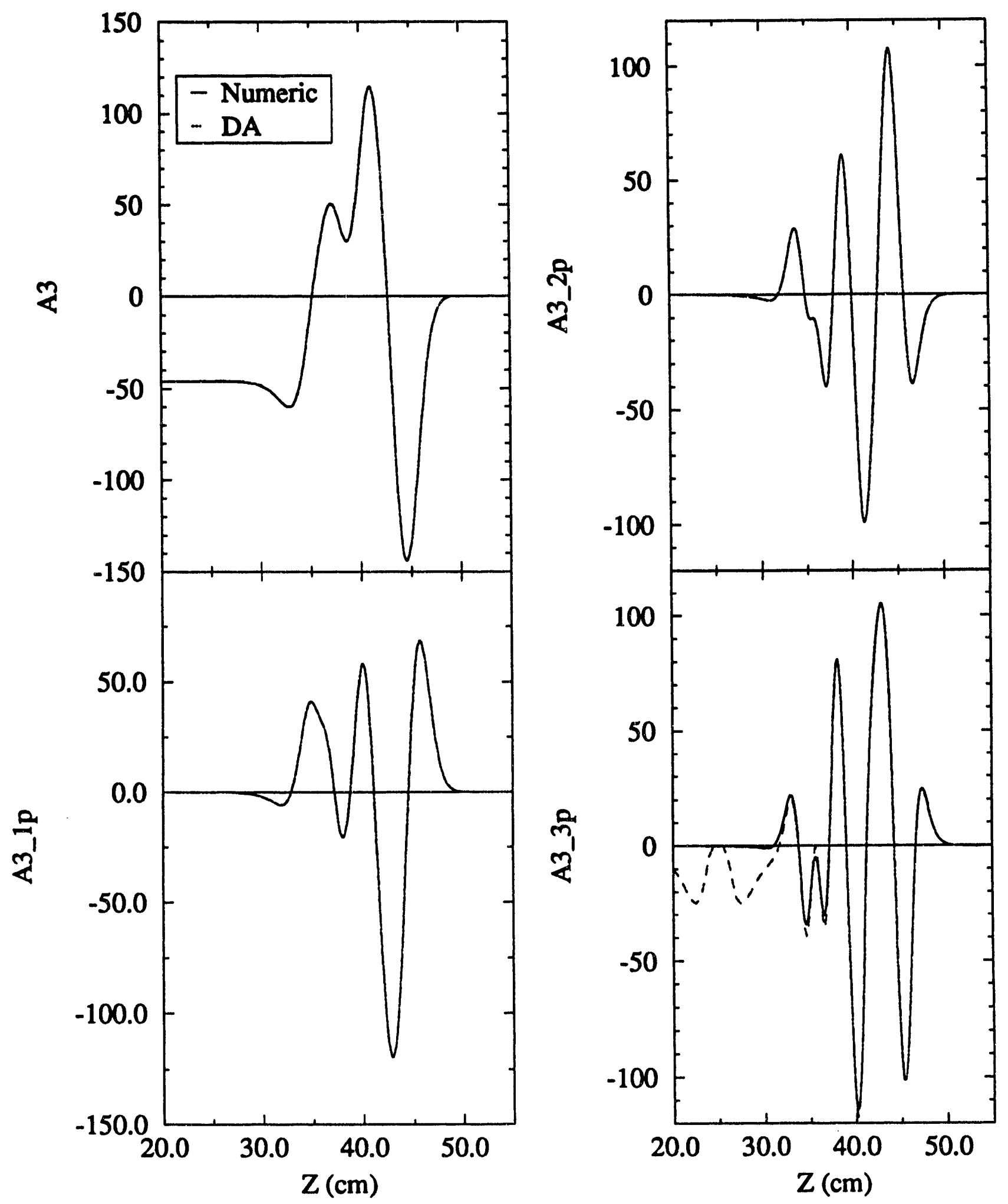

Figure 26 The SEXTUPOLE function ( $n=3$ ) A3(z) and pseudo harmonics $\frac{\partial A_{0}(z)}{\partial z}, \frac{\partial^{2} A_{3}(z)}{\partial z^{2}}, \frac{\theta^{3} A_{0}(z)}{\partial z^{3}}$. 

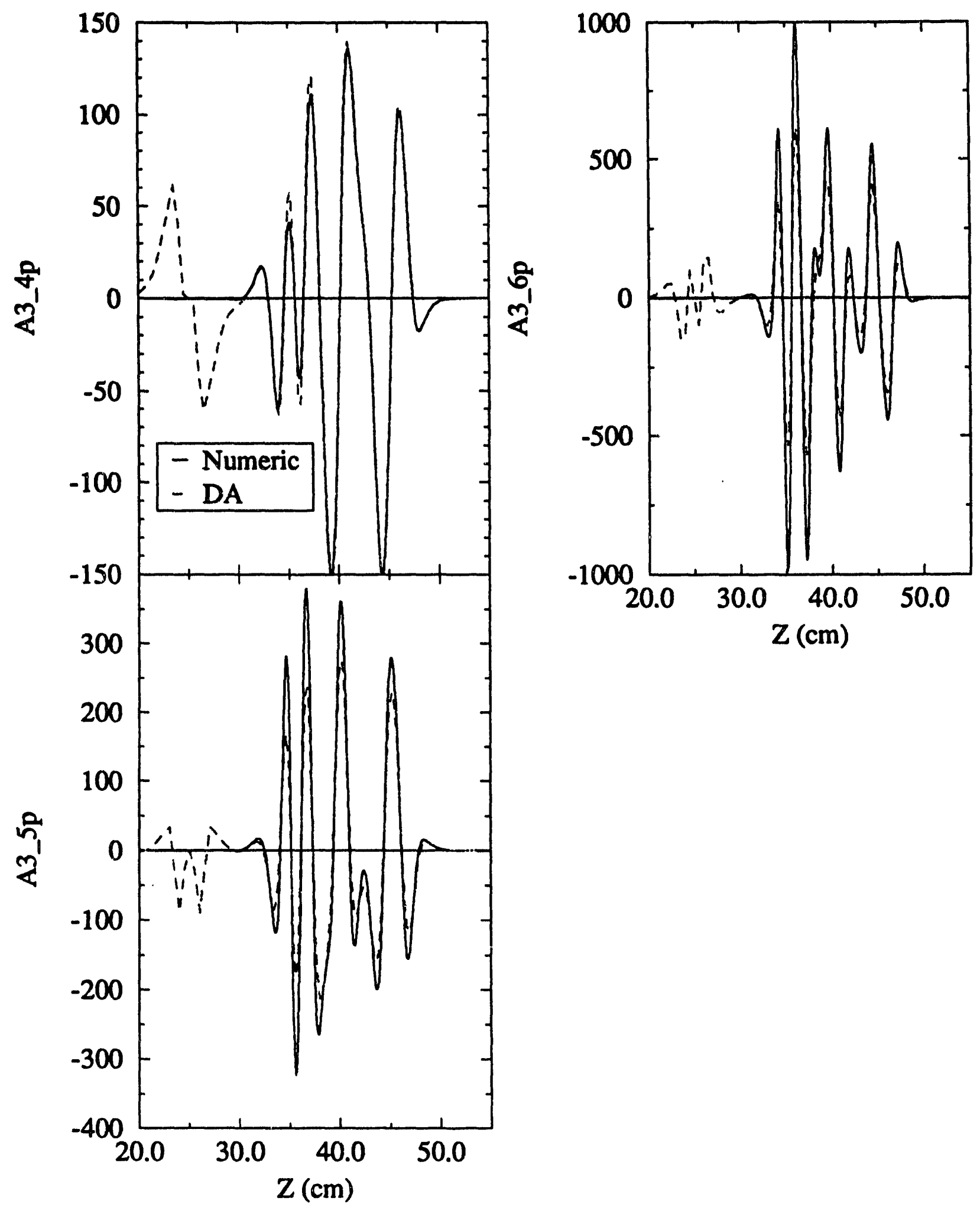

Figure 27 Pseudo harmonics of $A 3(z) \frac{\partial^{4} A_{3}(z)}{\partial x^{4}}, \frac{\partial^{8} A_{3}(z)}{\partial z^{3}}, \frac{\partial^{b} A_{3}(z)}{\partial z^{6}}$. 

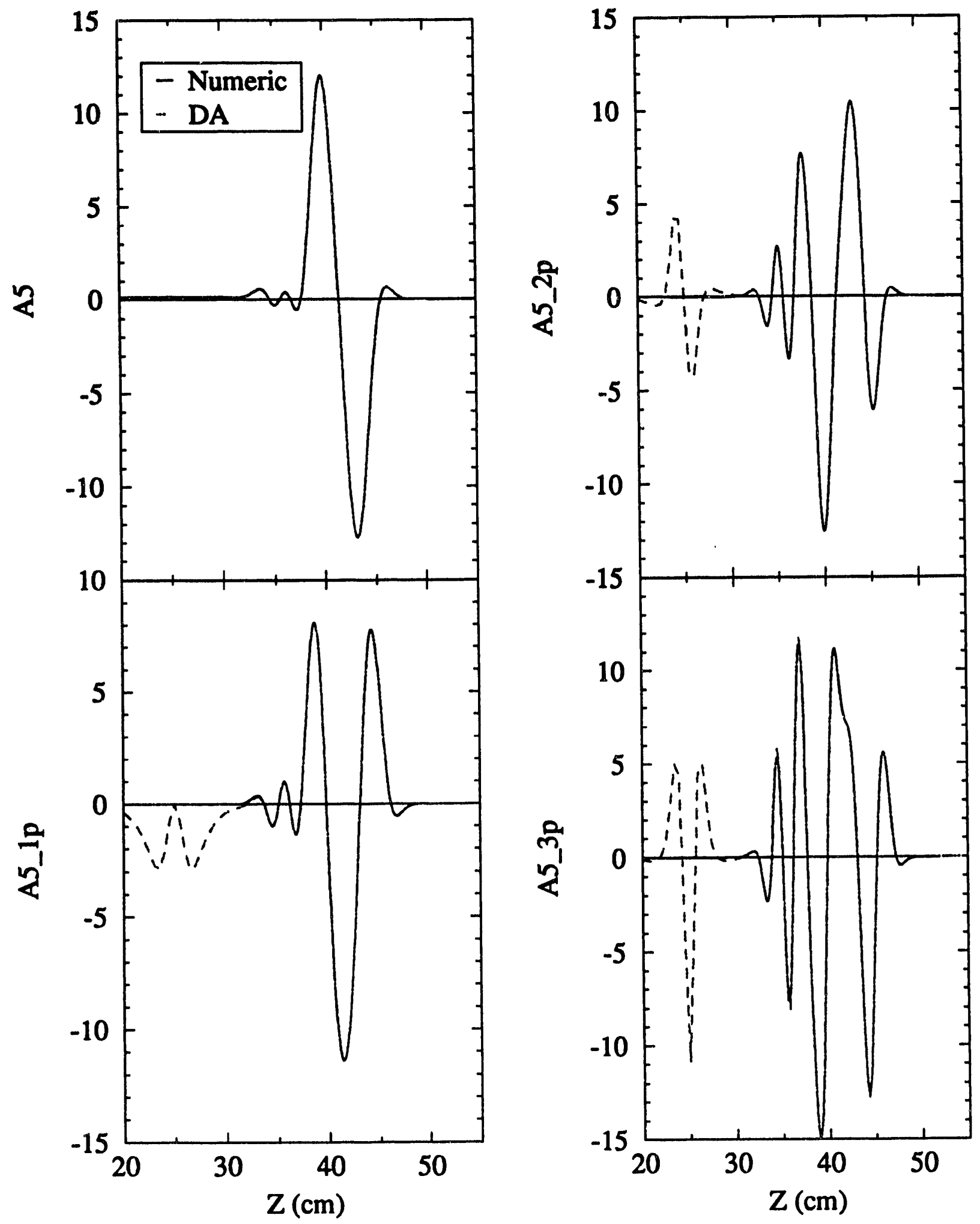

Figure 28 The DECAPOLE function ( $n=5)$ A5( $(z)$ and pseudo harmonics $\frac{\partial A_{5}(z)}{\partial z}, \frac{\partial^{2} A_{3}(z)}{\partial z^{2}}, \frac{\partial^{3} A_{5}(z)}{\partial z^{3}}$. 


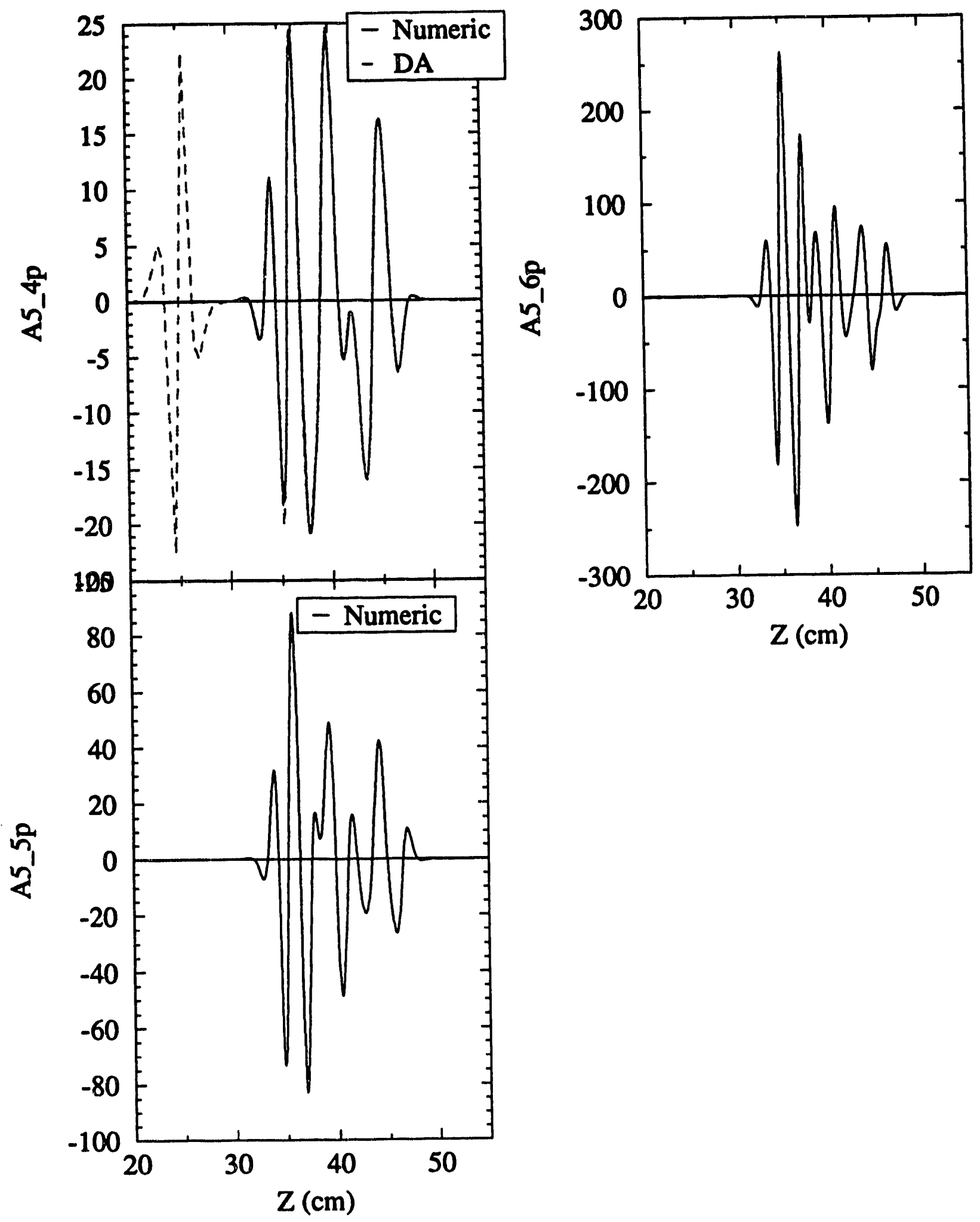

Figure 29 Pseudo harmonics of $A 5(z) \frac{\partial^{4} A_{3}(z)}{\partial z^{4}}, \frac{\theta^{8} A_{8}(z)}{\partial z^{b}}, \frac{\theta^{\theta} A_{5}(z)}{\theta_{z}^{b}}$. 

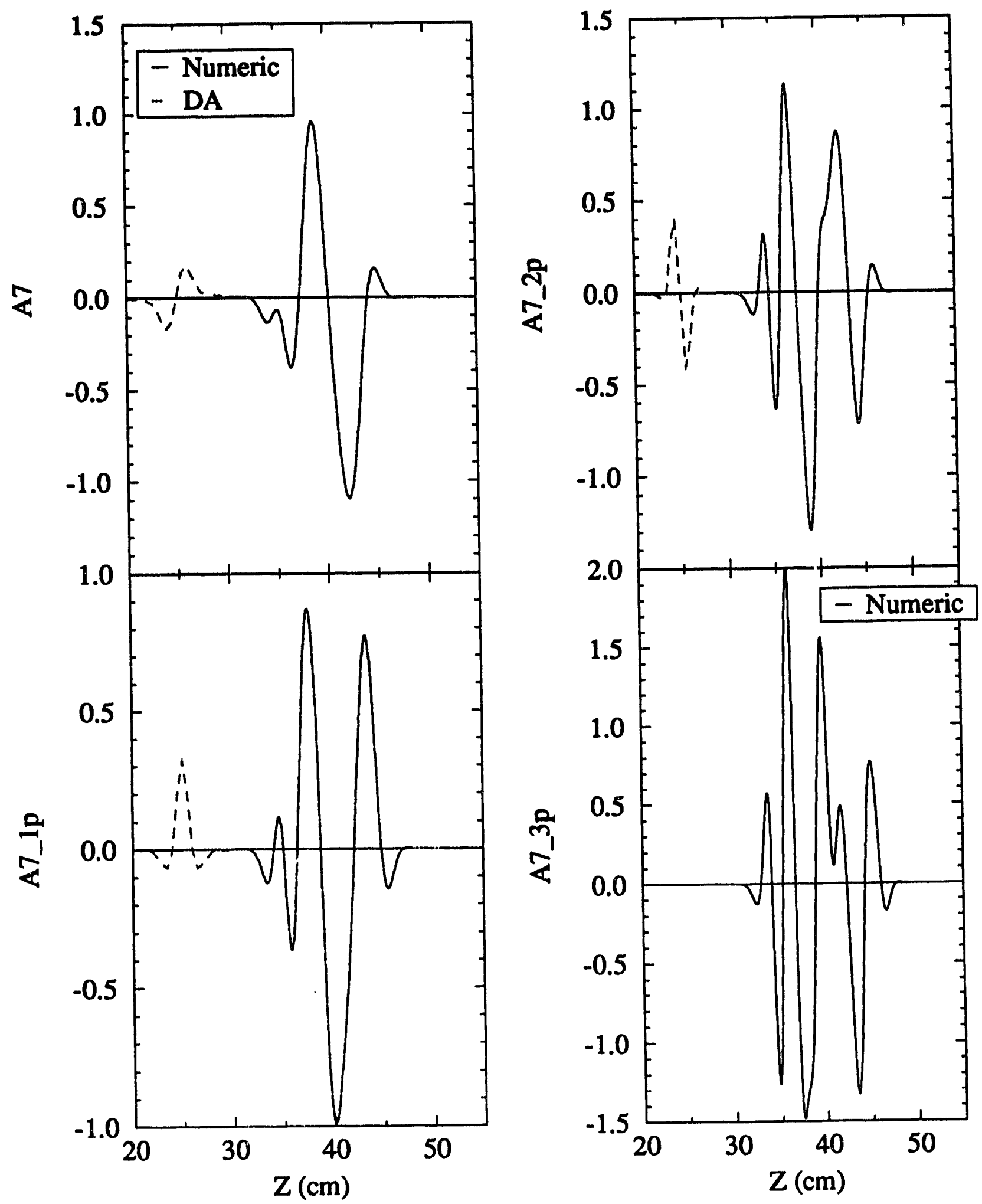

Figure 30 The 14 pole function ( $n=7$ ) A7(z) and pseudo harmonics $\frac{\partial A_{7}(z)}{\partial z}, \frac{\partial^{2} A_{7}(z)}{\partial z^{2}}, \frac{\partial^{3} A_{7}(z)}{\partial z^{3}}$. 


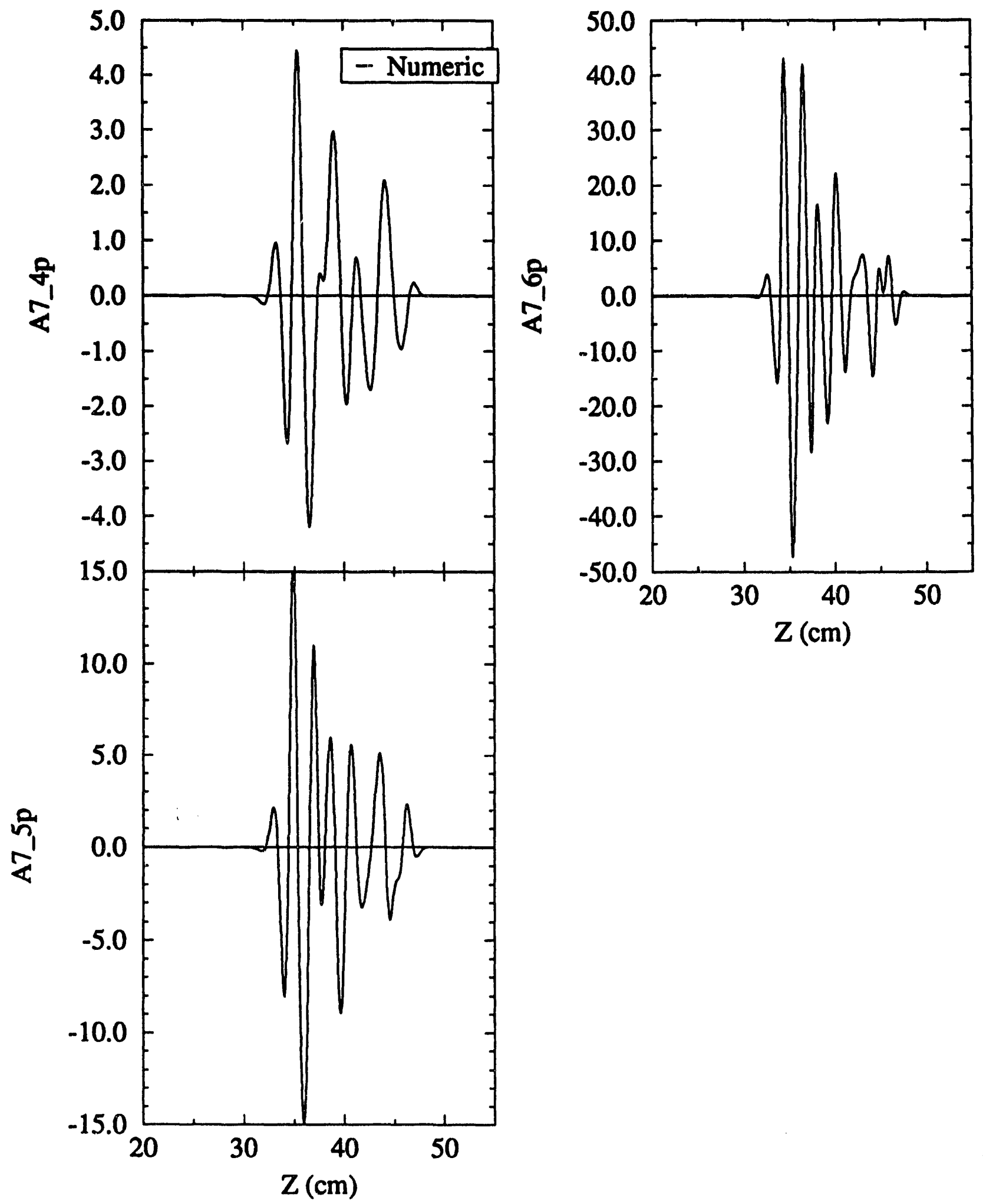

Figure 31 Pseudo harmonics of A7(z) $\frac{\partial^{4} A_{7}(z)}{\partial z^{4}}, \frac{\theta^{8} A_{7}(z)}{\partial z^{b}}, \frac{\theta^{8} A_{7}(z)}{\partial z^{6}}$. 

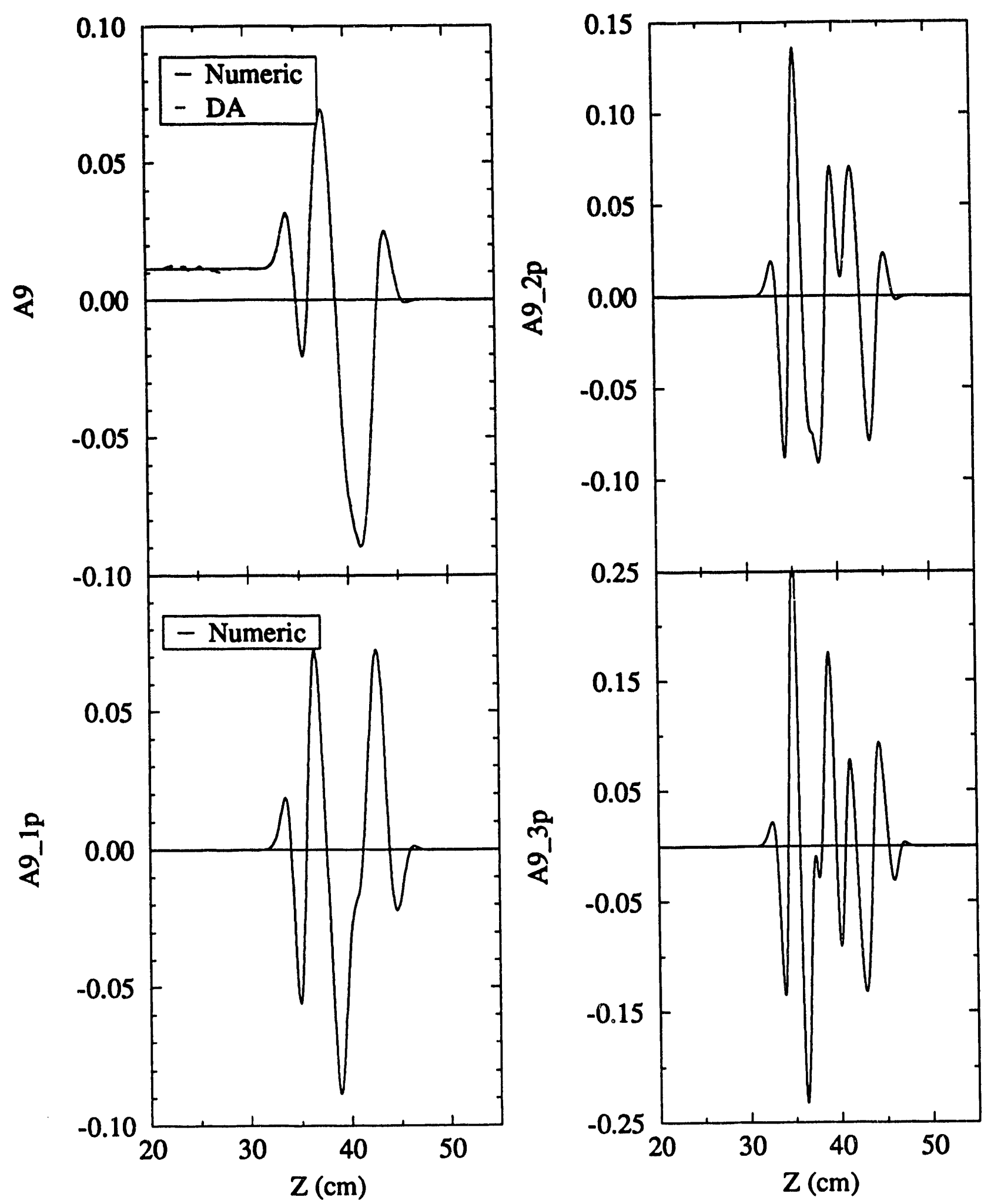

Figure 32 The 18 pole function ( $n=9)$ A9(z) and pseudo harmonics $\frac{\partial A_{g}(z)}{\partial z}, \frac{\partial^{2} A_{9}(z)}{\partial z^{2}}, \frac{\partial^{3} A_{g}(z)}{\partial z^{3}}$. 


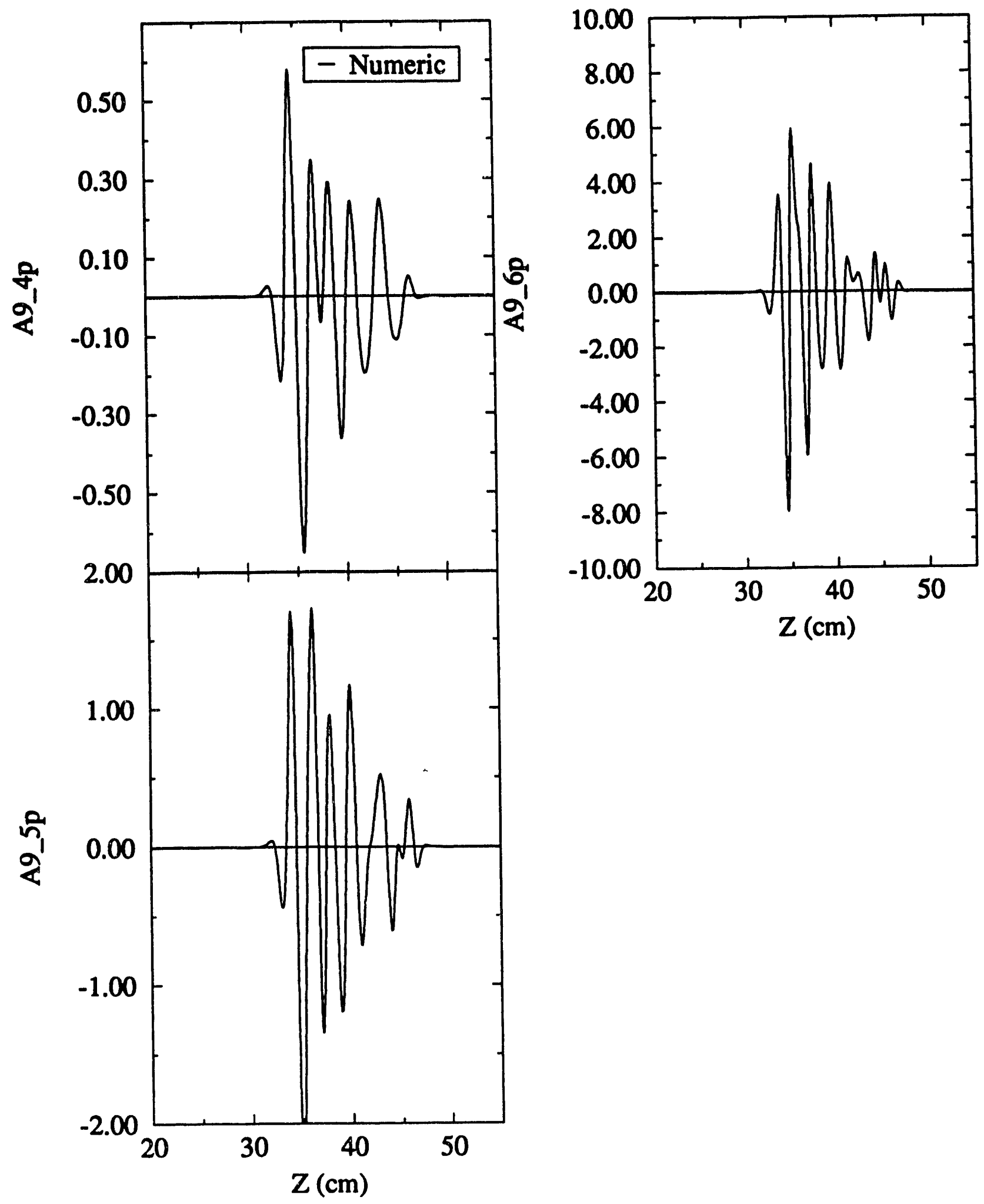

Figure 33 Pseudo harmonics of $A 9(z) \frac{\partial^{4} A_{0}(z)}{\partial z^{6}}, \frac{\theta^{5} A_{p}(z)}{\partial z^{b}}, \frac{\partial^{8} A_{2}(z)}{\partial z^{6}}$. 

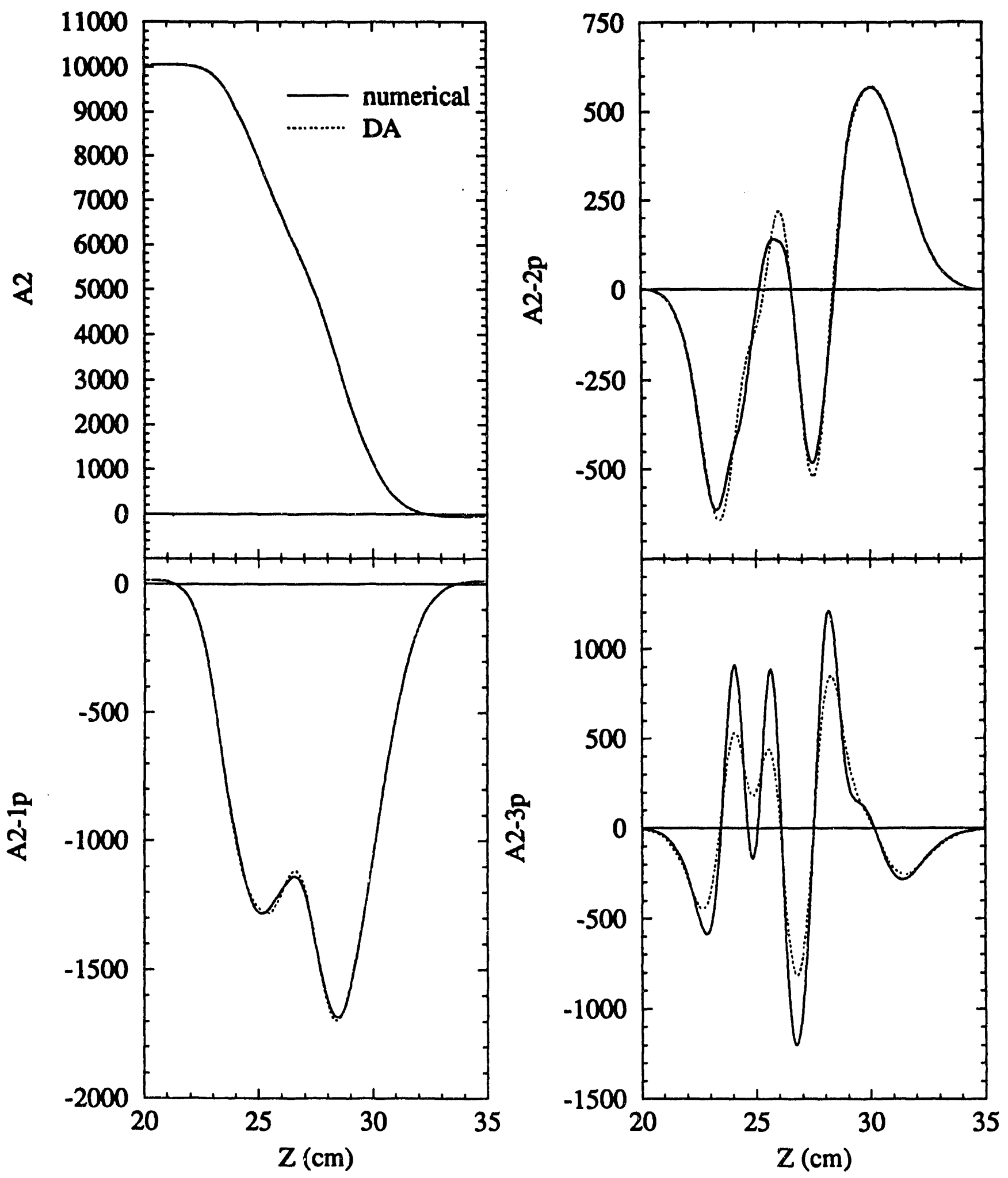

Figure 34 The QUAD function ( $n=2)$ A2(z) and pseudo harmonics $\frac{\partial A_{2}(z)}{\partial z}, \frac{\partial^{2} A_{2}(z)}{\partial z^{2}}, \frac{\partial^{3} A_{2}(z)}{\partial z^{3}}$. 

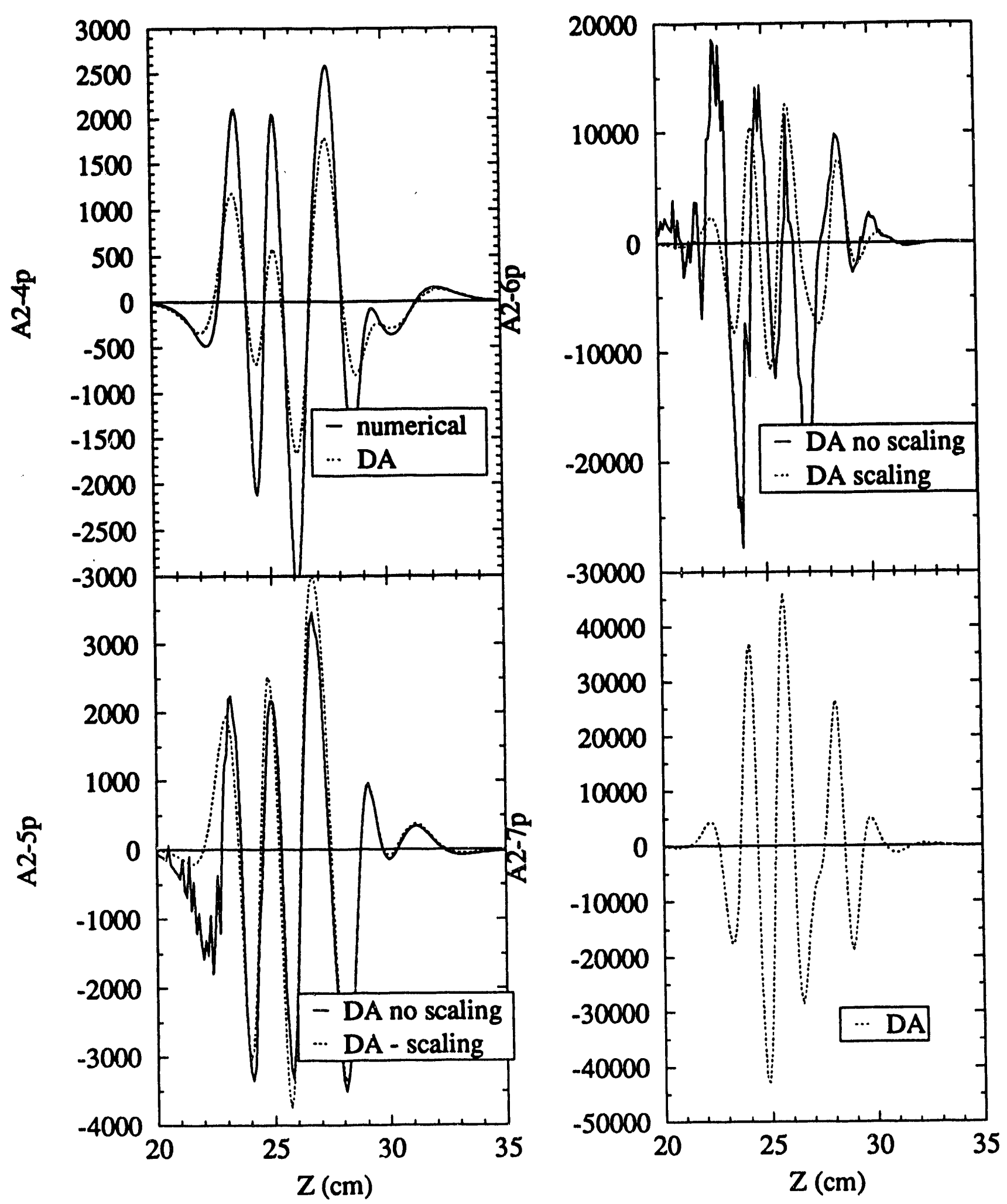

Figure 35 Pseudo harmonics of A2(z) $\frac{\partial^{4} A_{2}(z)}{\partial z^{4}}, \frac{\partial^{8} A_{2}(z)}{\partial z^{s}}, \frac{\partial^{8} A_{2}(z)}{\partial z^{6}}, \frac{\theta^{7} A_{2}(z)}{\partial z^{7}}$. 


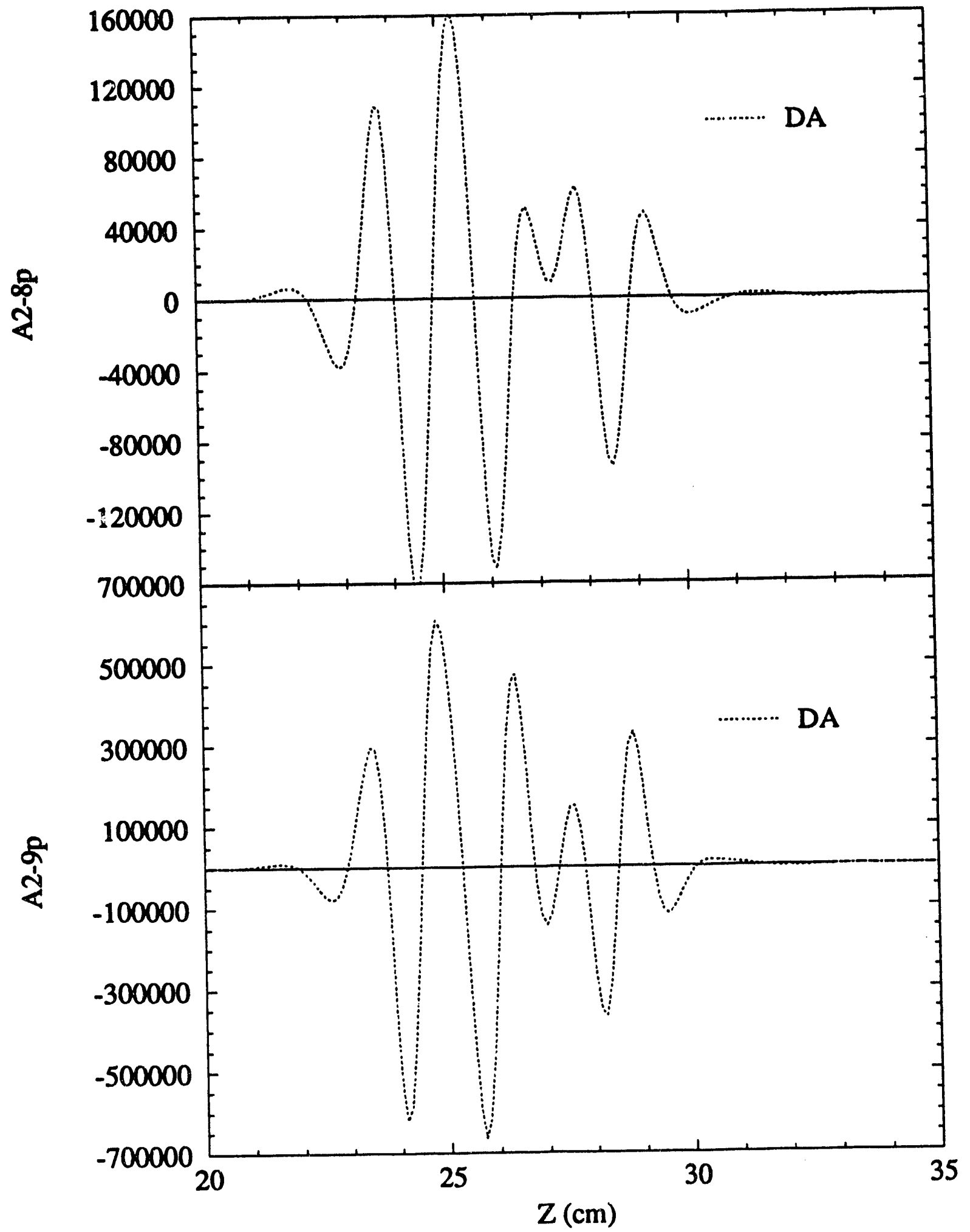

Figure 36 Pseudo harmonics of $A 2(z) \frac{\partial^{8} A_{2}(z)}{\partial z^{b}}, \frac{\partial^{D} A_{2}(z)}{\partial z^{b}}$. 

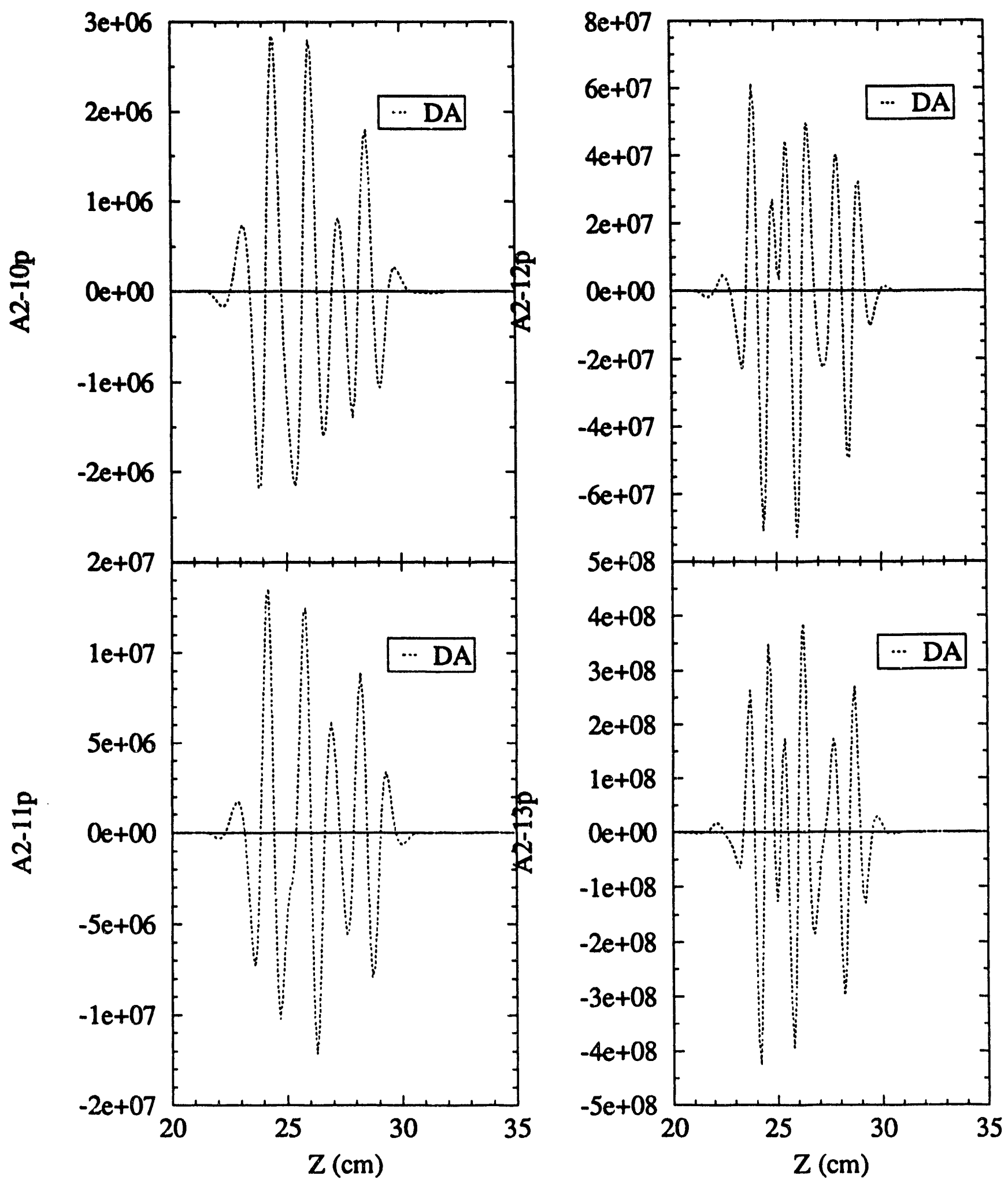

Figure 37 Pseudo harmonics of A2(z) $\frac{\partial^{10} A_{2}(z)}{\partial z^{10}}, \frac{\partial^{11} A_{2}(z)}{\partial z^{11}}, \frac{\partial^{12} A_{2}(z)}{\partial z^{13}}, \frac{\partial^{19} A_{2}(z)}{\partial z^{13}}$. 


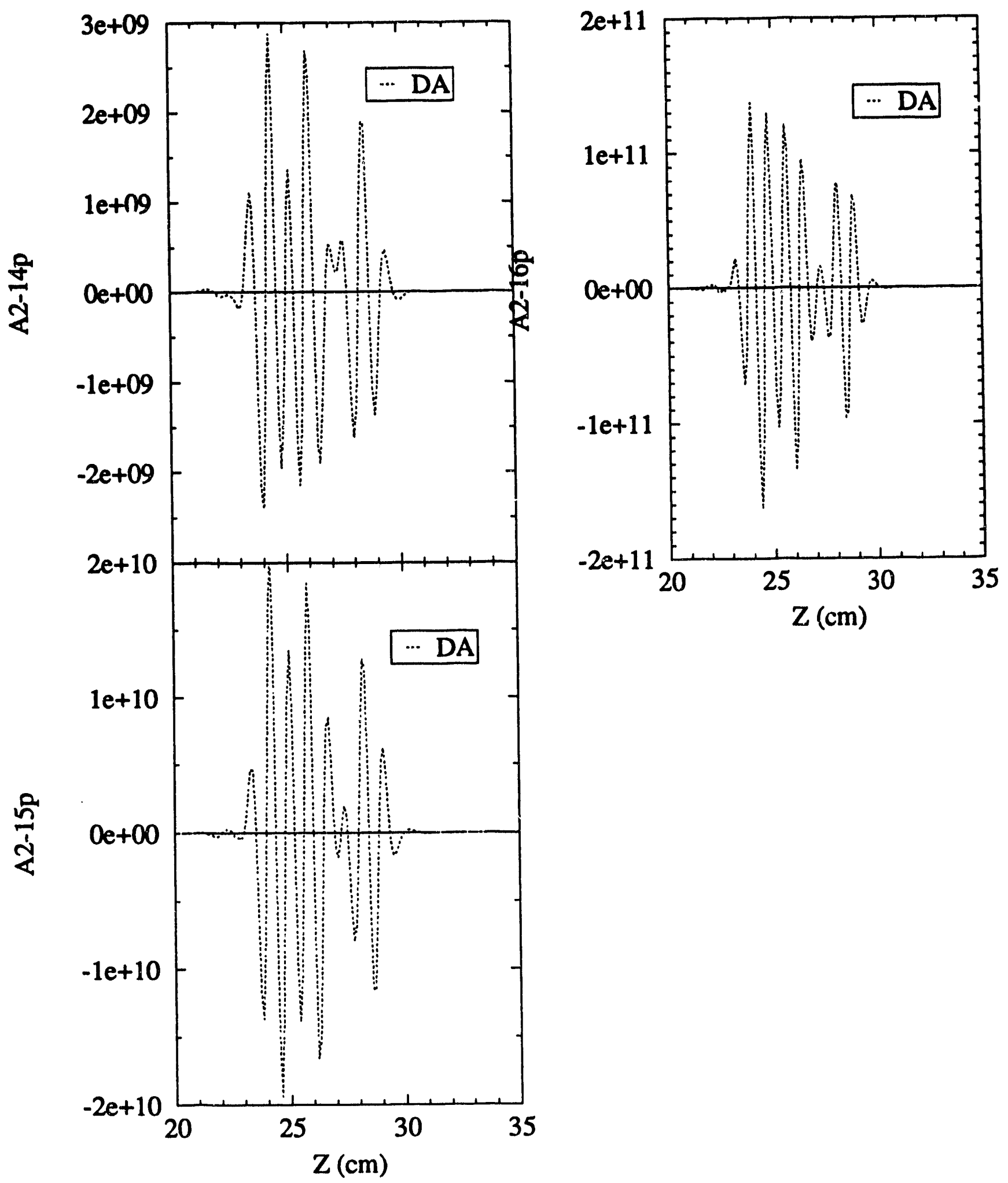

Figure 38 Pseudo harmonics of A2(z) $\frac{\theta^{14} A_{2}(z)}{\partial z^{14}}, \frac{\theta^{15} A_{z}(z)}{\partial z^{15}}, \frac{\theta^{16} A_{z}(z)}{\partial z^{16}}$. 

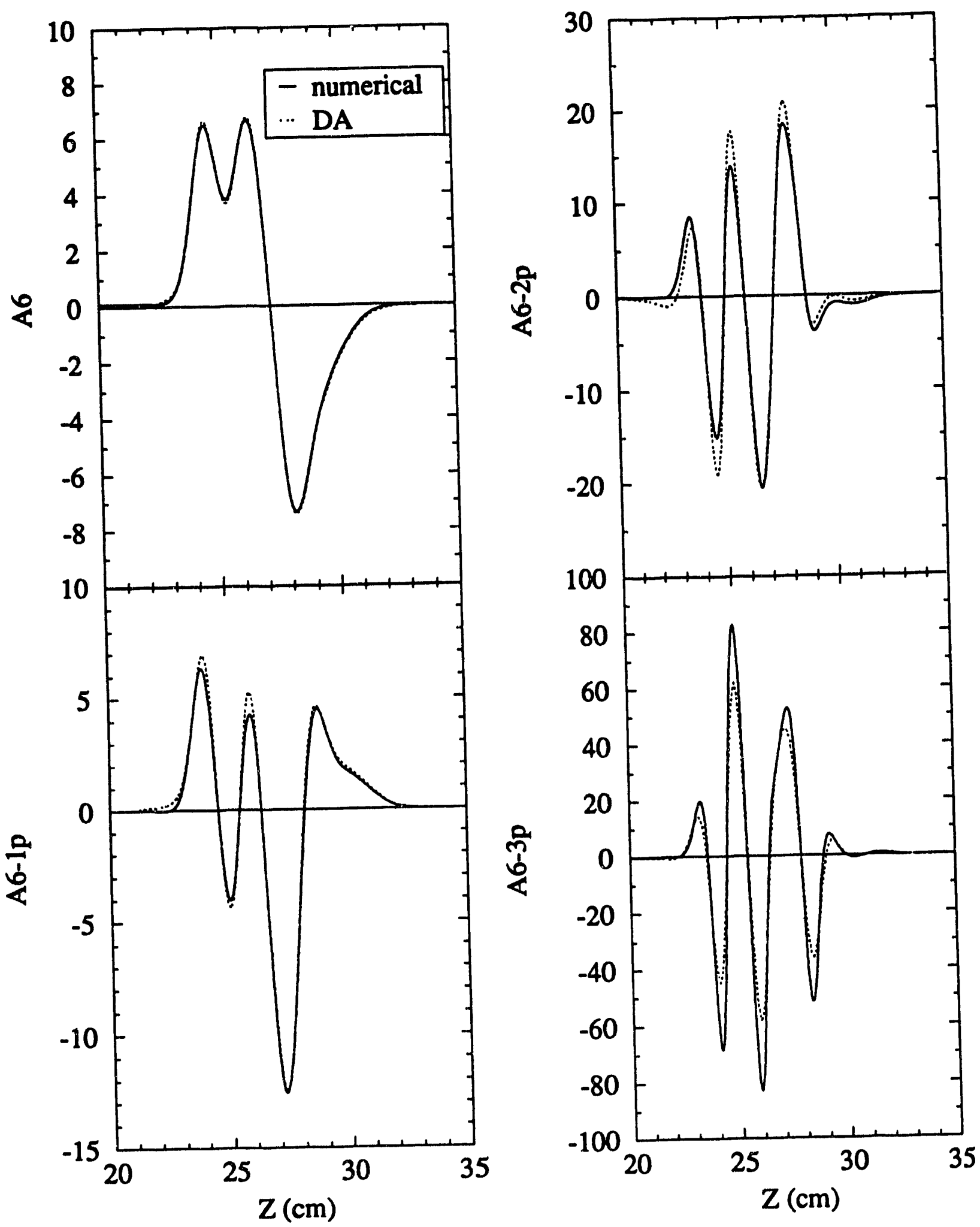

Figure 39 The dodecapole function $(n=6) A 6(z)$ and pseudo harmonics $\frac{\theta A_{6}(z)}{\partial z}, \frac{\theta^{2} A_{6}(z)}{\partial z^{2}}, \frac{\partial^{3} A_{8}(z)}{\partial z^{3}}$. 

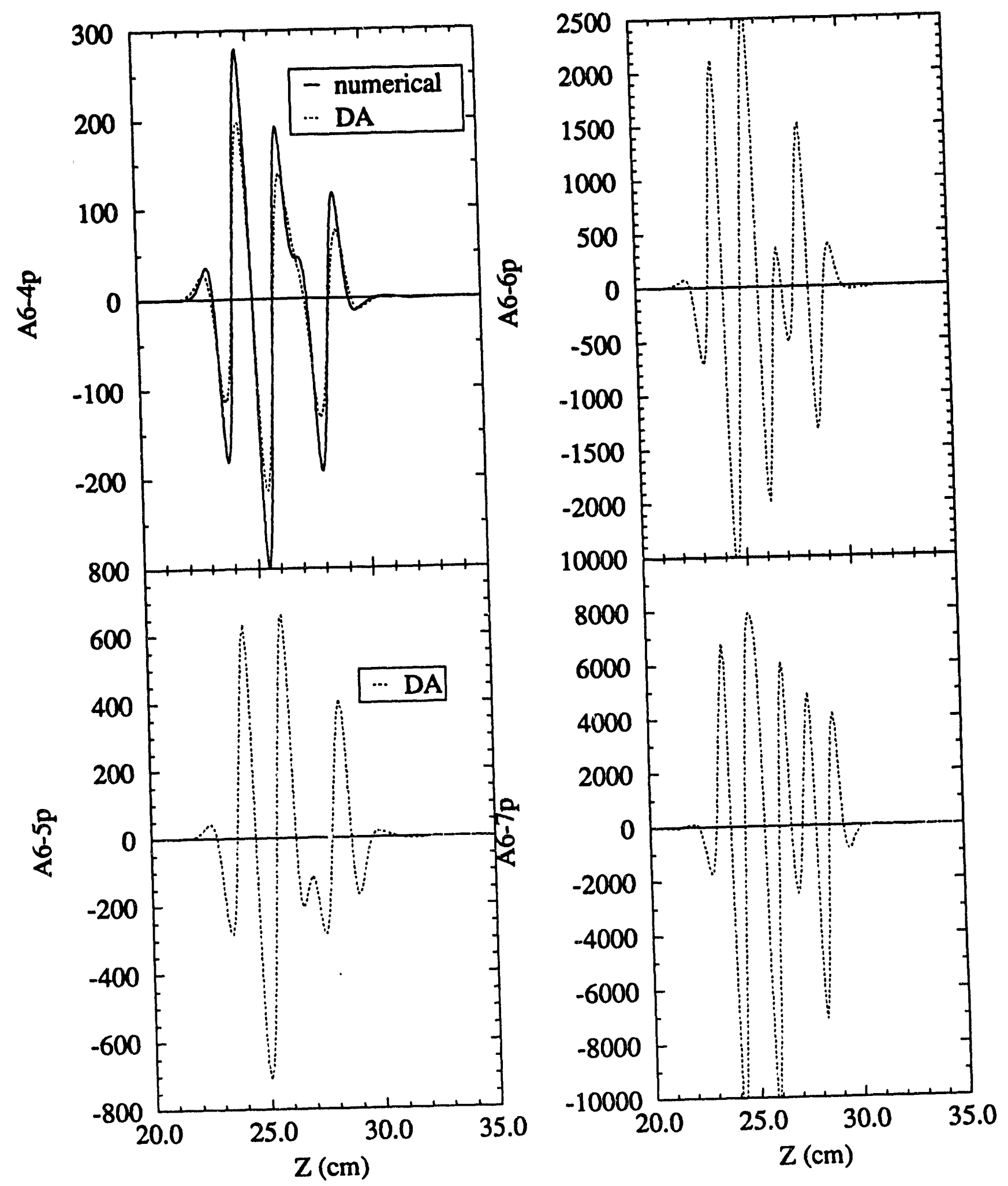

Figure 40 Pseudo harmonics of A6(z) $\frac{\partial^{4} A_{8}(z)}{\partial z^{4}}, \frac{\partial^{8} A_{0}(z)}{\partial z^{3}}, \frac{\partial^{8} A_{8}(z)}{\partial z^{6}}, \frac{\partial^{7} A_{8}(z)}{\partial z^{7}}$. 


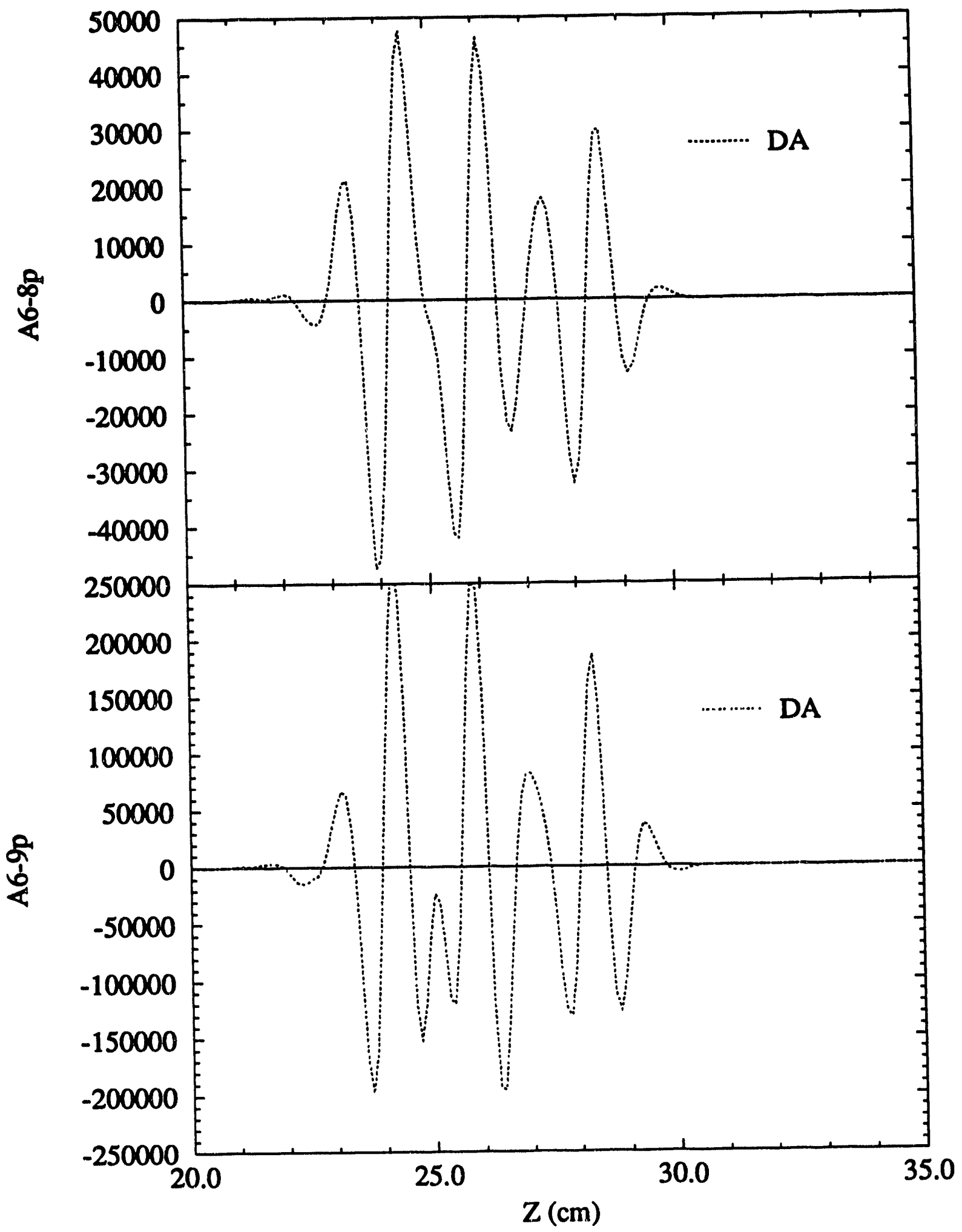

Figure 41 Pseudo harmonics of $A 6(z) \frac{\theta^{\theta} A_{0}(z)}{\theta_{z^{b}}}, \frac{\theta^{\theta} A_{0}(z)}{\partial z^{\theta}}$. 


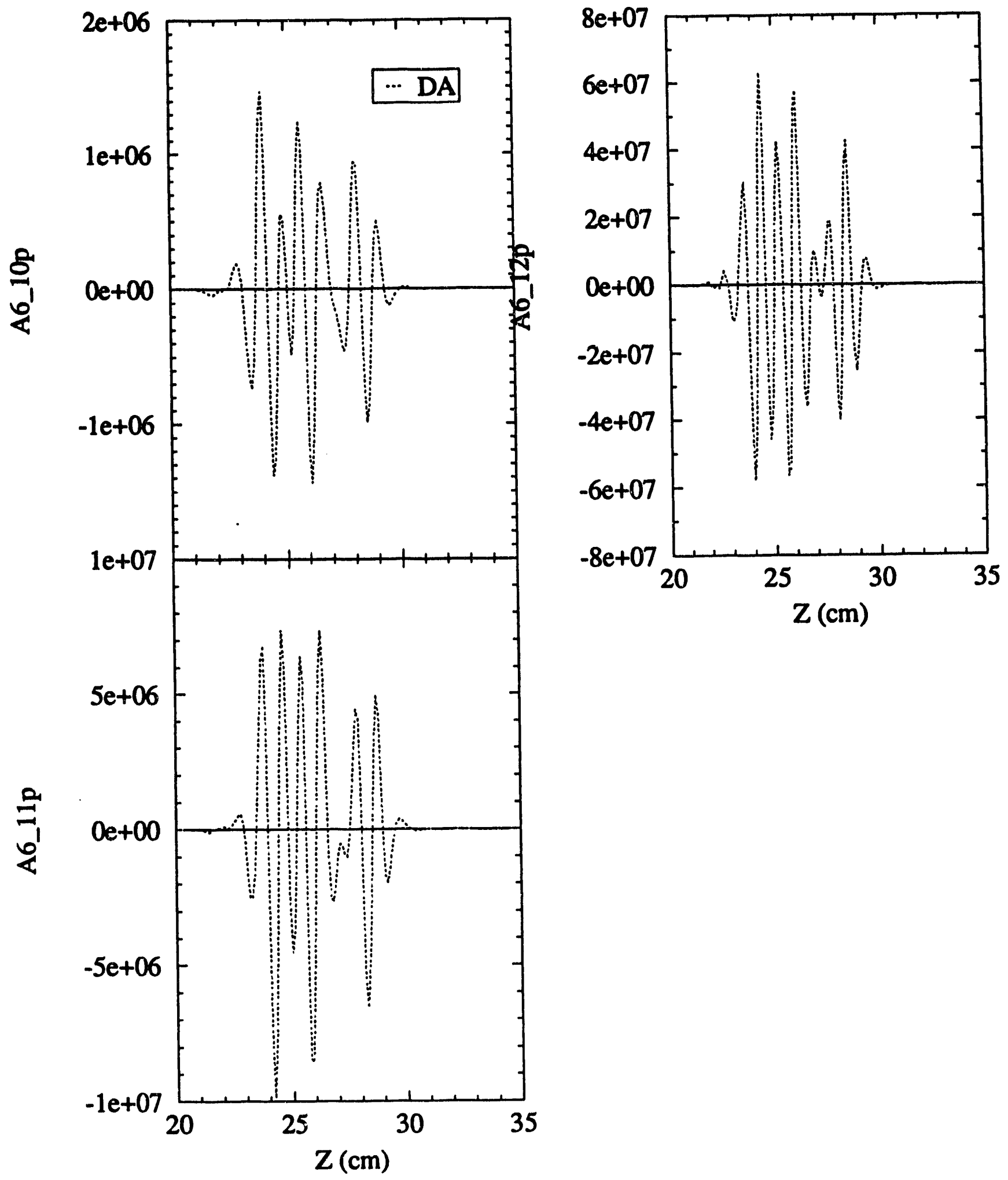

Figure 42 Pseudo harmonics of $A 6(z) \frac{\theta^{10} A_{8}(z)}{\theta_{z}^{10}}, \frac{\theta^{11} A_{6}(z)}{\theta_{z}^{11}}, \frac{\partial^{12} A_{g}(z)}{\partial z^{12}}$. 

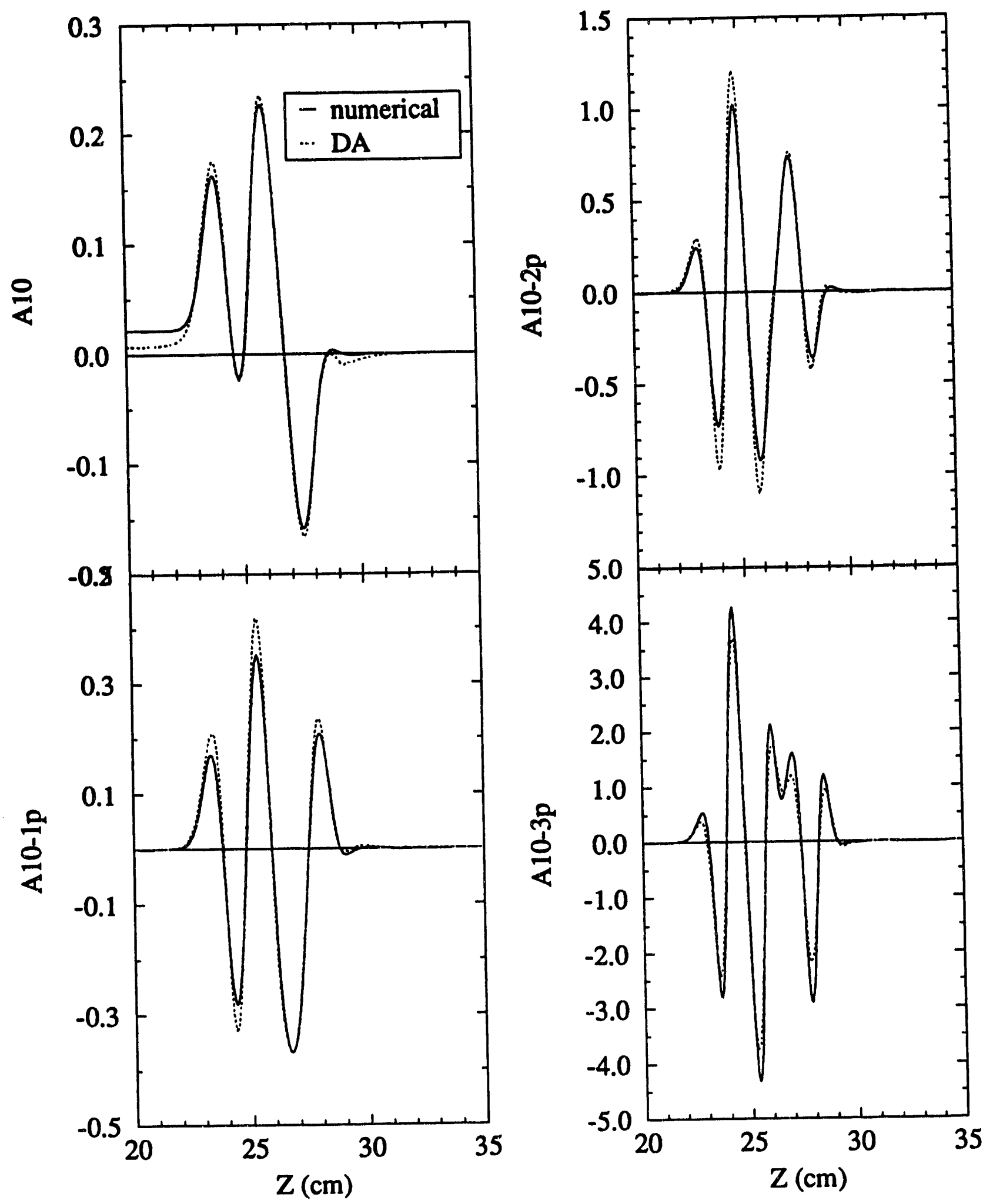

Figure 43 The 20 pole function ( $n=10$ ) A1O(z) and pseudo harmonics $\frac{\partial A_{10}(z)}{\partial z}, \frac{\partial^{2} A_{10}(z)}{\partial z^{2}}, \frac{\theta^{3} A_{10}(z)}{\partial z^{3}}$. 

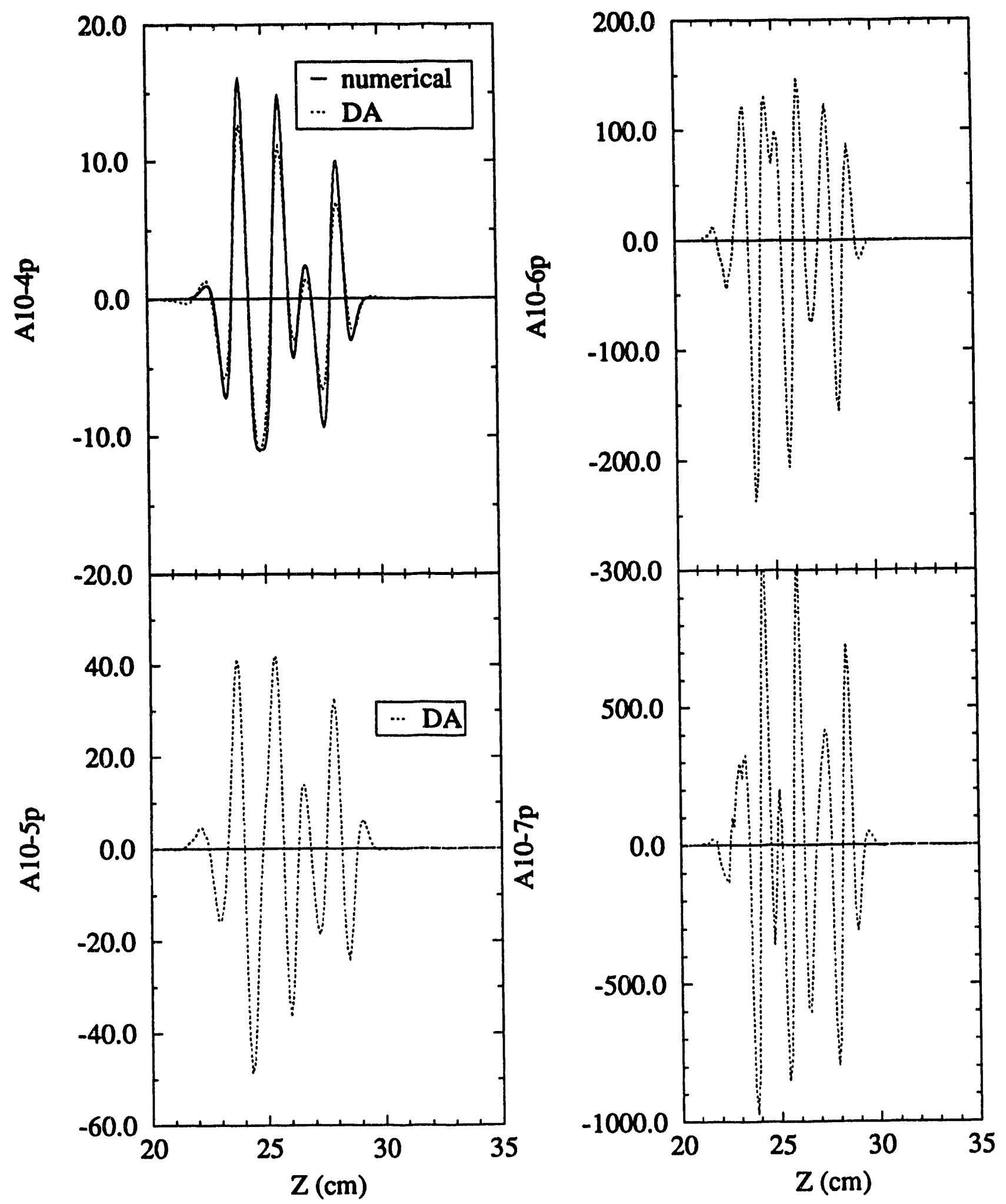

Figure 44 Pseudo harmonics of $A 10(z) \frac{\partial^{4} A_{10}(z)}{\partial z^{4}}, \frac{\partial^{8} A_{20}(z)}{\partial z^{8}}, \frac{\theta^{8} A_{10}(z)}{\partial z^{6}}, \frac{\partial^{7} A_{10}(z)}{\partial z^{7}}$. 


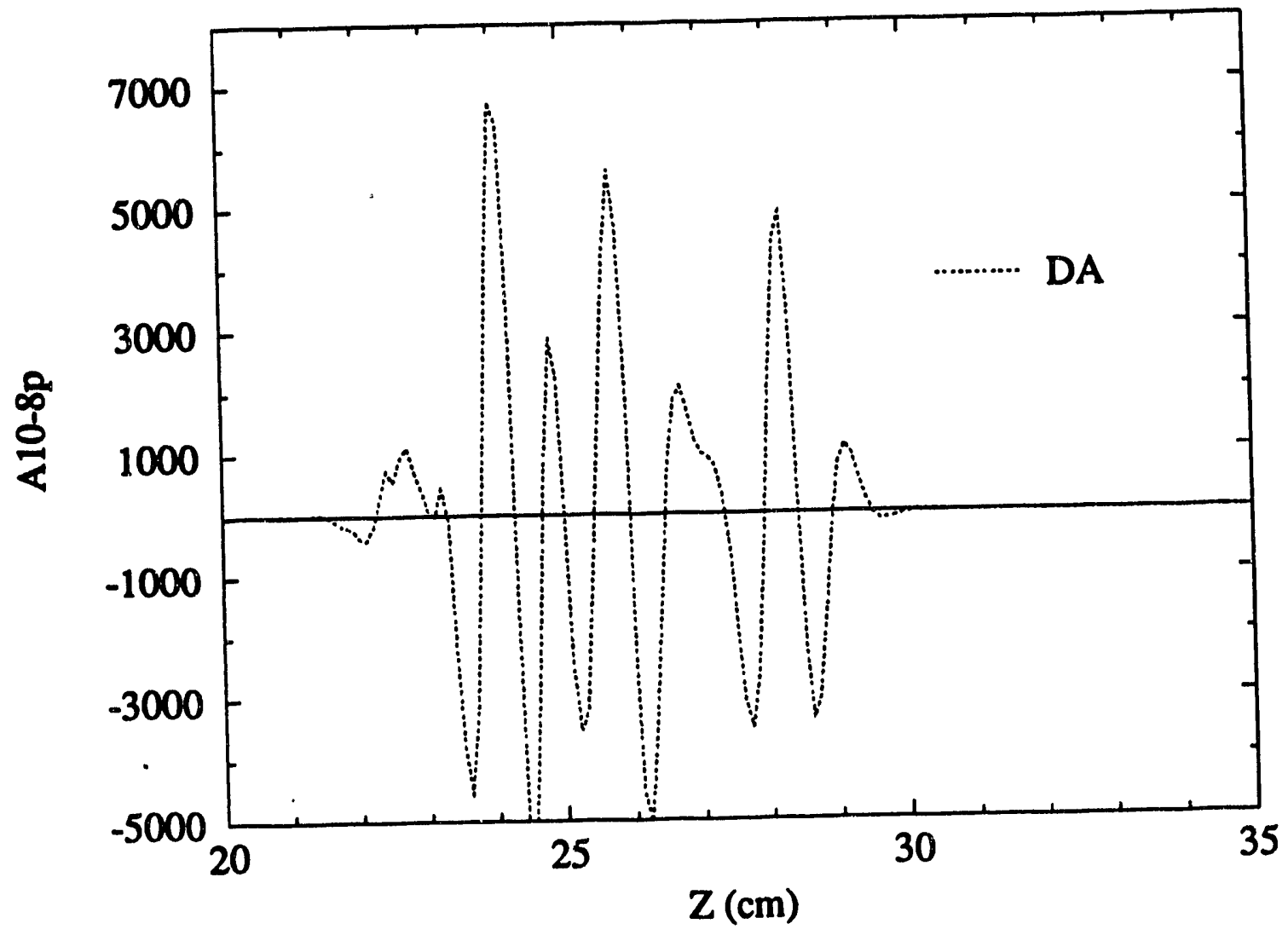

Figure 45 Pseudo harmonics of $A 10(z) \frac{\theta^{0} A_{10}(z)}{\partial_{2} z^{3}}$. 

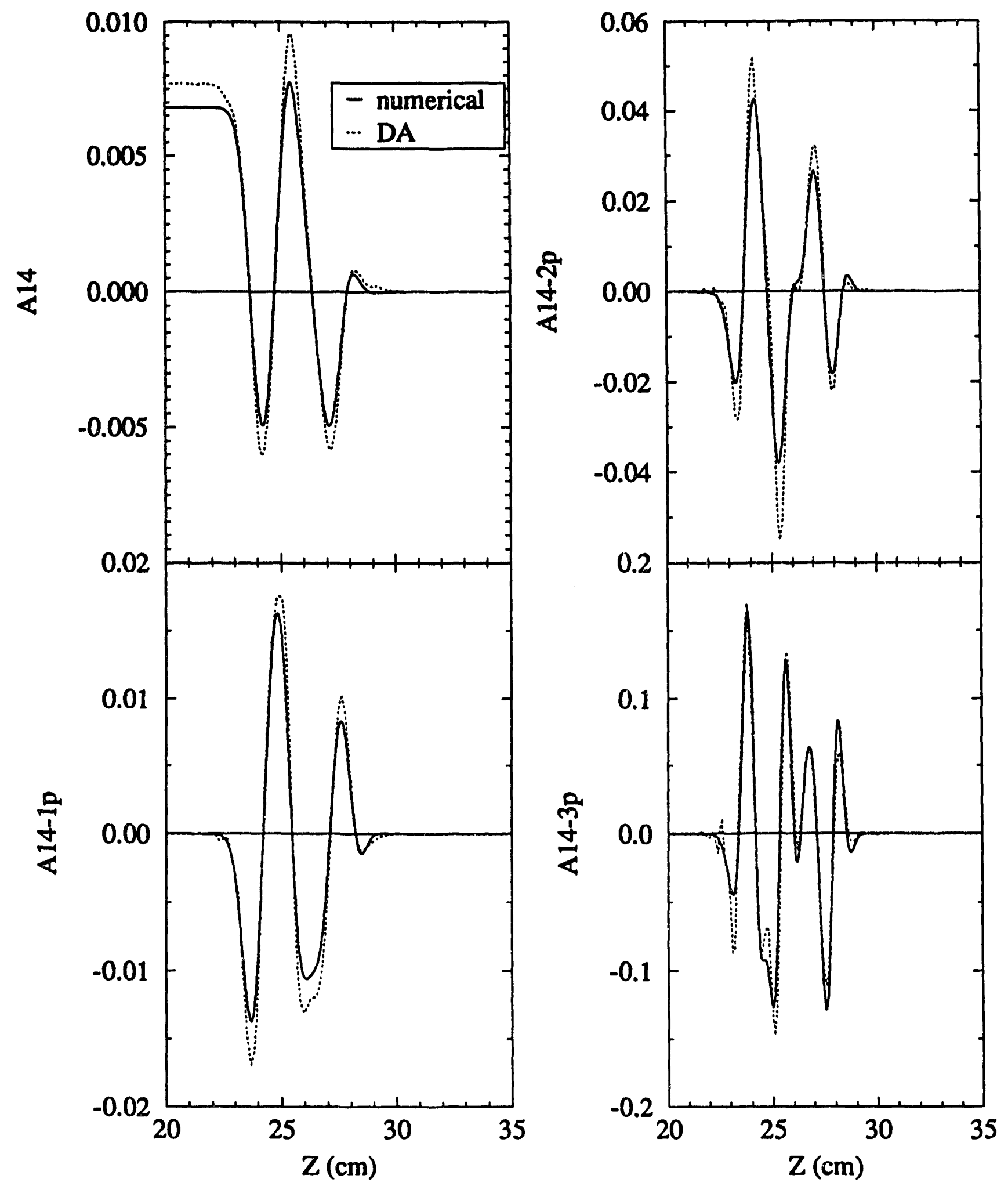

Figure 46 The 28 pole function ( $n=14$ ) A14(z) and pseudo harmonics $\frac{\partial A_{14}(z)}{\partial z}, \frac{\partial^{2} A_{11}(z)}{\partial z^{2}}, \frac{\partial^{3} A_{14}(z)}{\partial z^{3}}$. 


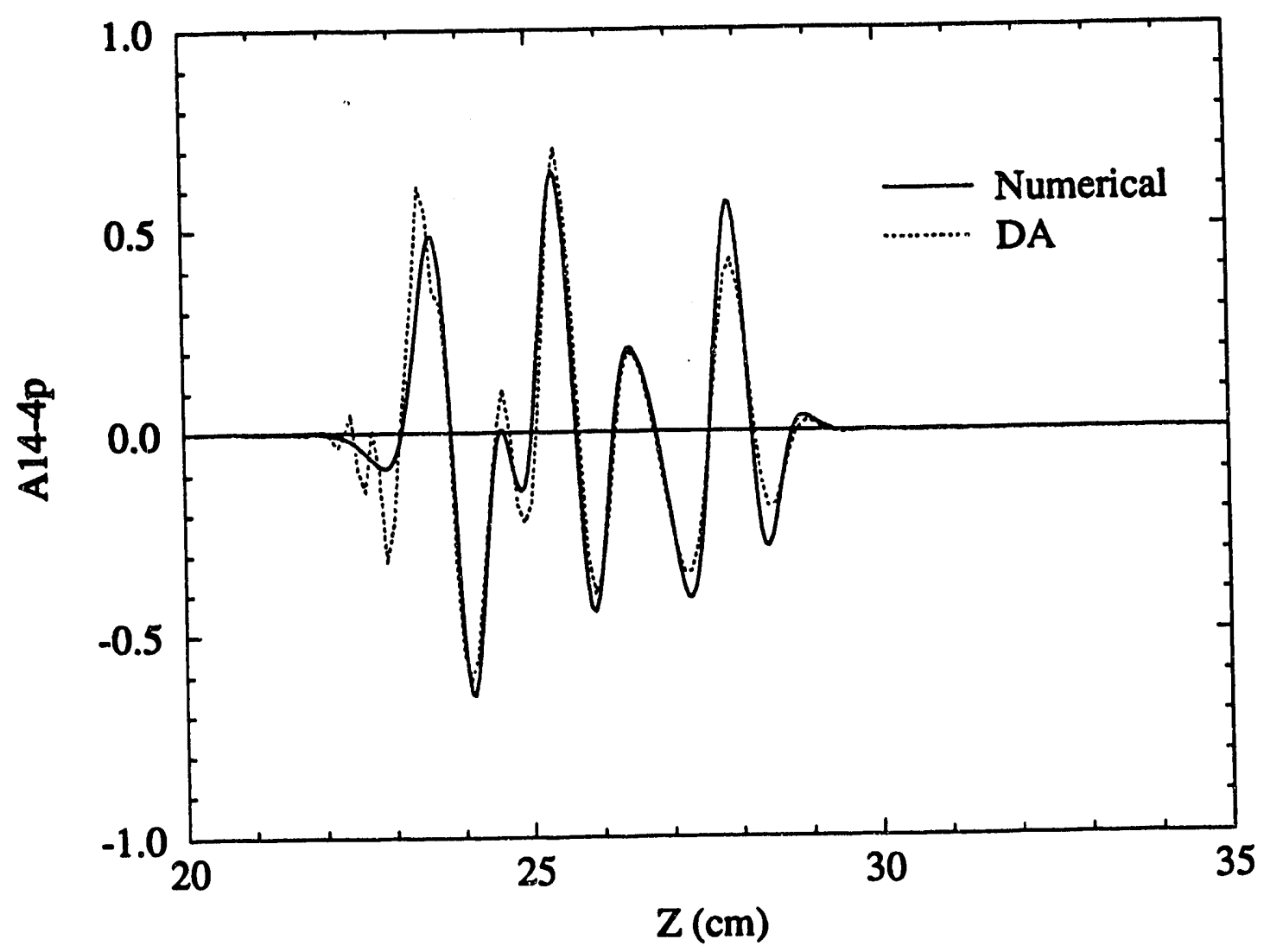

Figure 47 Pseudo harmonics of A14(z) $\frac{\theta^{4} A_{14}(z)}{\partial z^{4}}$. 

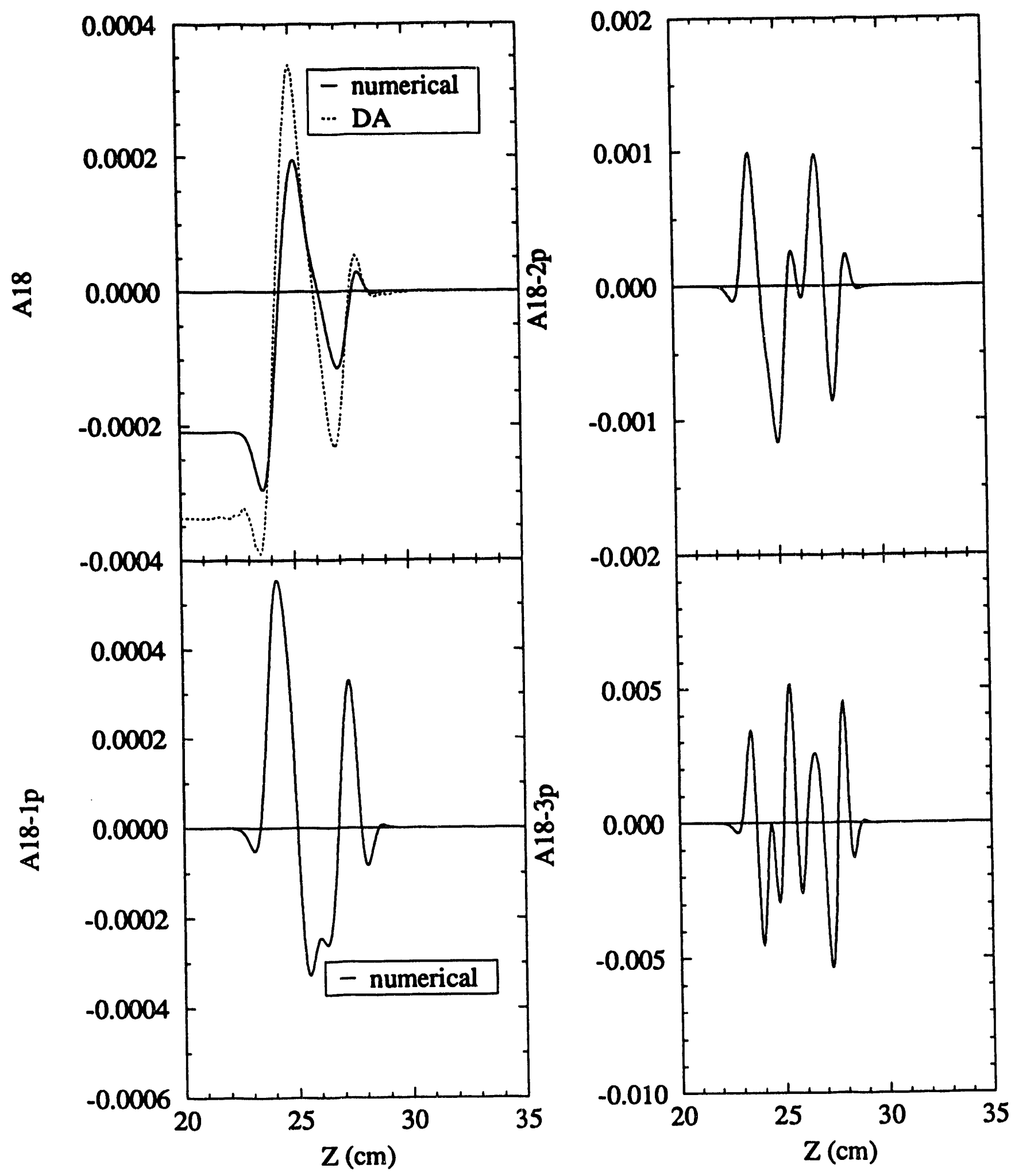

Figure 48 The 36 pole function ( $n=18$ ) A18(z) and pseudo harmonics $\frac{\partial A_{11}(z)}{\partial z}, \frac{\partial^{2} A_{1}(z)}{\partial z^{2}}, \frac{\partial^{3} A_{11}(z)}{\partial z^{3}}$. 


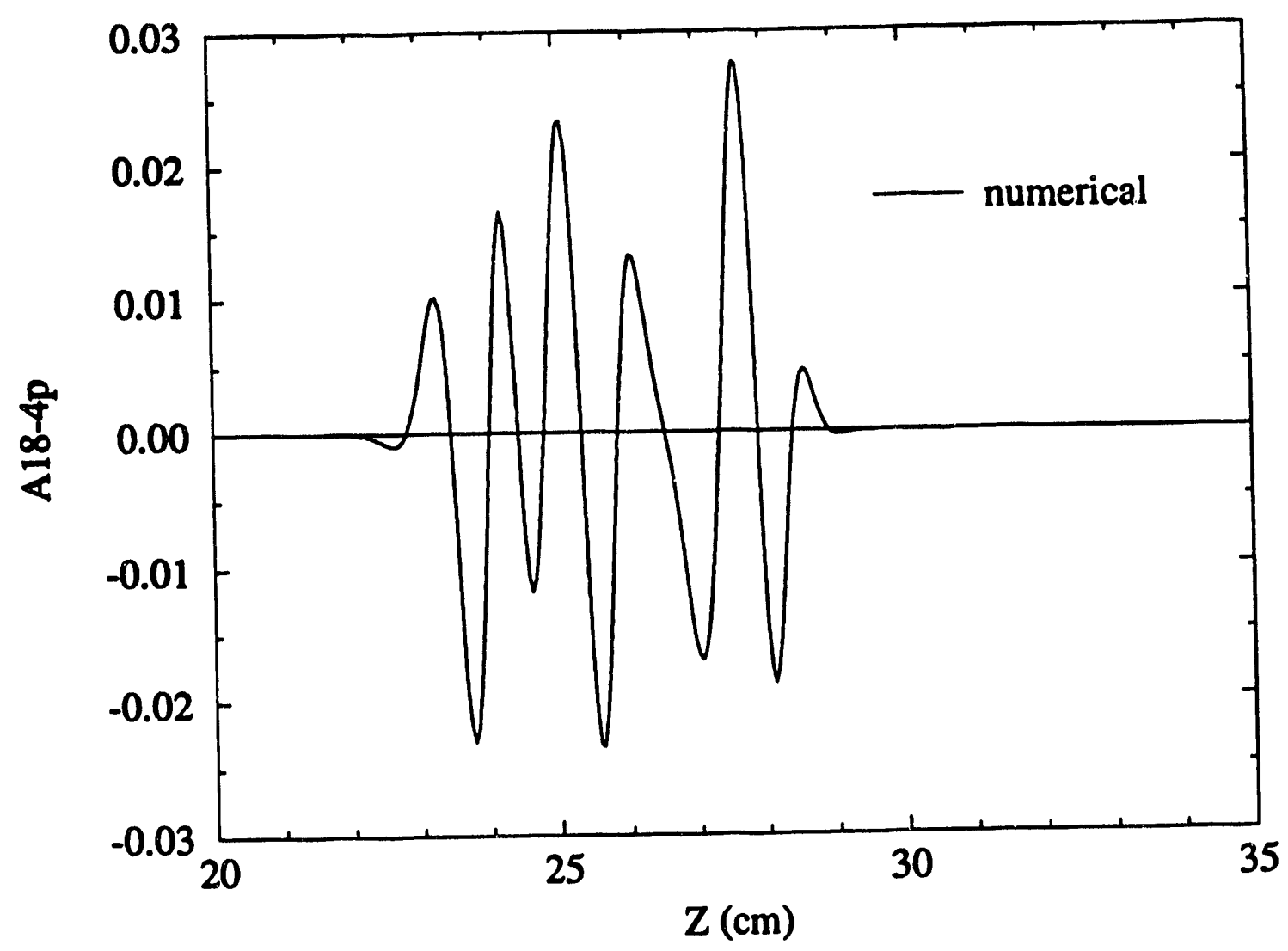

Figure 49 Pseudo harmonics of $A 18(z) \frac{\partial^{4} A_{1}(z)}{\partial z^{t}}$. 


\section{Appendix A Biot-Savart and DA}

c \$Header: dapkg.fox,v 1.1 91/10/14 17:48:47 mike Locked \$

c \$Log: dapkg.fox, v \$

c Revision 1.1 91/10/14 17:48:47 mike

c Initial revision

C

c

subroutine dapkg( xyza, xyzb, xff, cwir, bbst,

\$ $x f, y f, z f$, by )

c

implicit double precision $(a-h, 0-z)$

c

double precision xyza(3), xyzb(3), xff(3), cwir, bbst(3)

double precision $x a, y a, z a, x b, y b, z b, b x, b z$

common no, nv

integer no, nv

c

$\star F O X$ B D :

*FOX D V DA EXT XF NO NV ;

*FOX D V DA EXT YF NO NV ;

*FOX D V DA EXT ZF NO NV;

*FOX D V DA EXT BY NO NV ;

*FOX D V RE EXT XYZA 3 ;

*FOX D V RE EXT XYZB 3 ;

*FOX D V RE EXT XFF 3 ;

*FOX D V RE EXT CWIR ;

*FOX D V RE EXT BBST 3 ;

*FOX D V RE INT XA :

${ }^{\star}$ FOX D V RE INT YA ;

*FOX D V RE INT ZA ;

*FOX D V RE INT XB;

*FOX D V RE INT YB ;

*FOX D V RE INT ZB ;

*FOX D V RE INT BX ;

${ }^{\star}$ FOX D V RE INT BZ;

*FOX E D ;

$c$

*DA $\quad X A=X Y Z A(1)$;

$\star D A \quad Y A=X Y Z A(2) ;$

$\star D A \quad Z A=X Y Z A(3)$;

$\star D A \quad X B=X Y Z B(1)$;

$\star D A \quad Y B=X Y Z B(2)$;

${ }^{*} \mathrm{DA} \quad \mathrm{ZB}=\mathrm{XYZB}(3)$; 


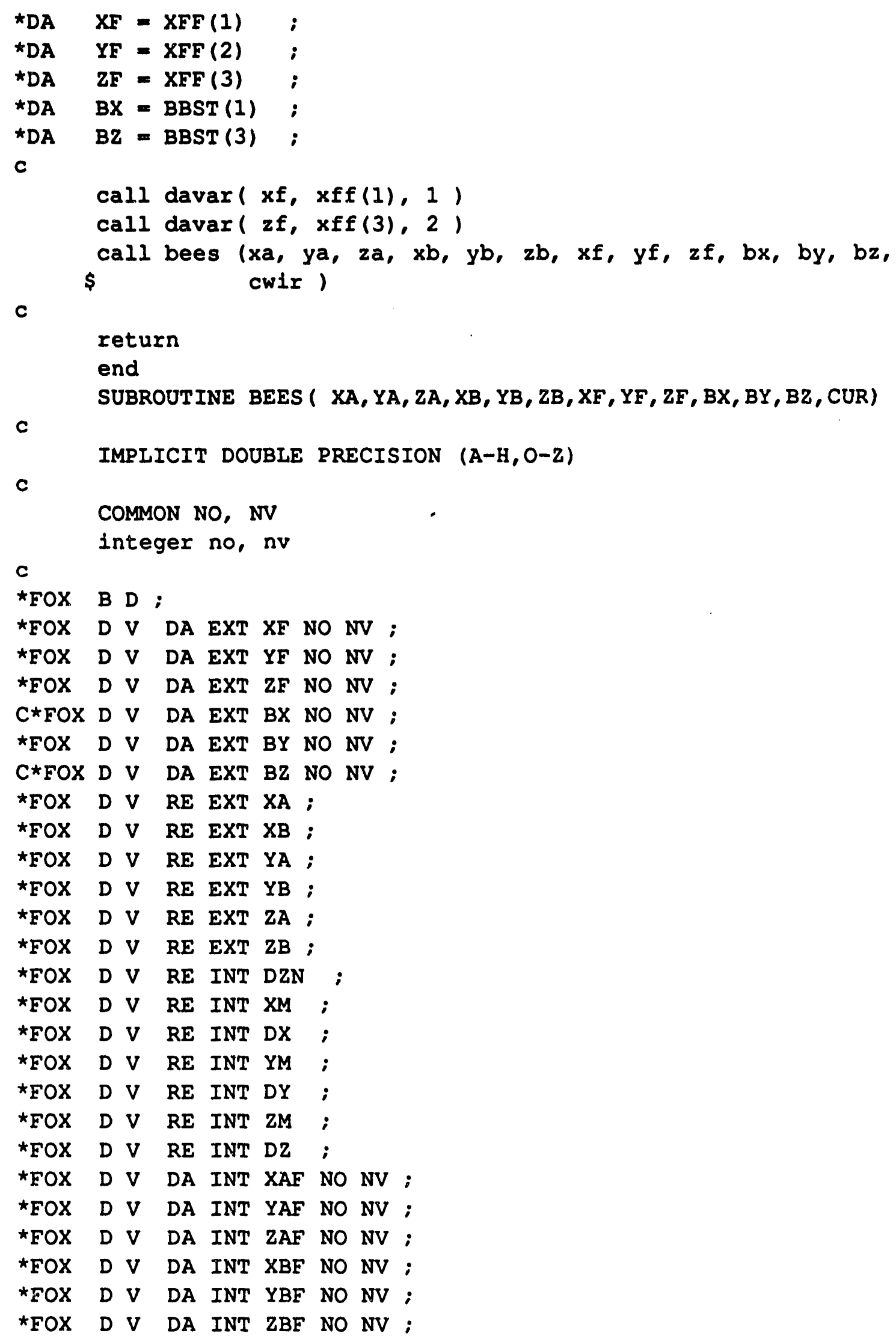




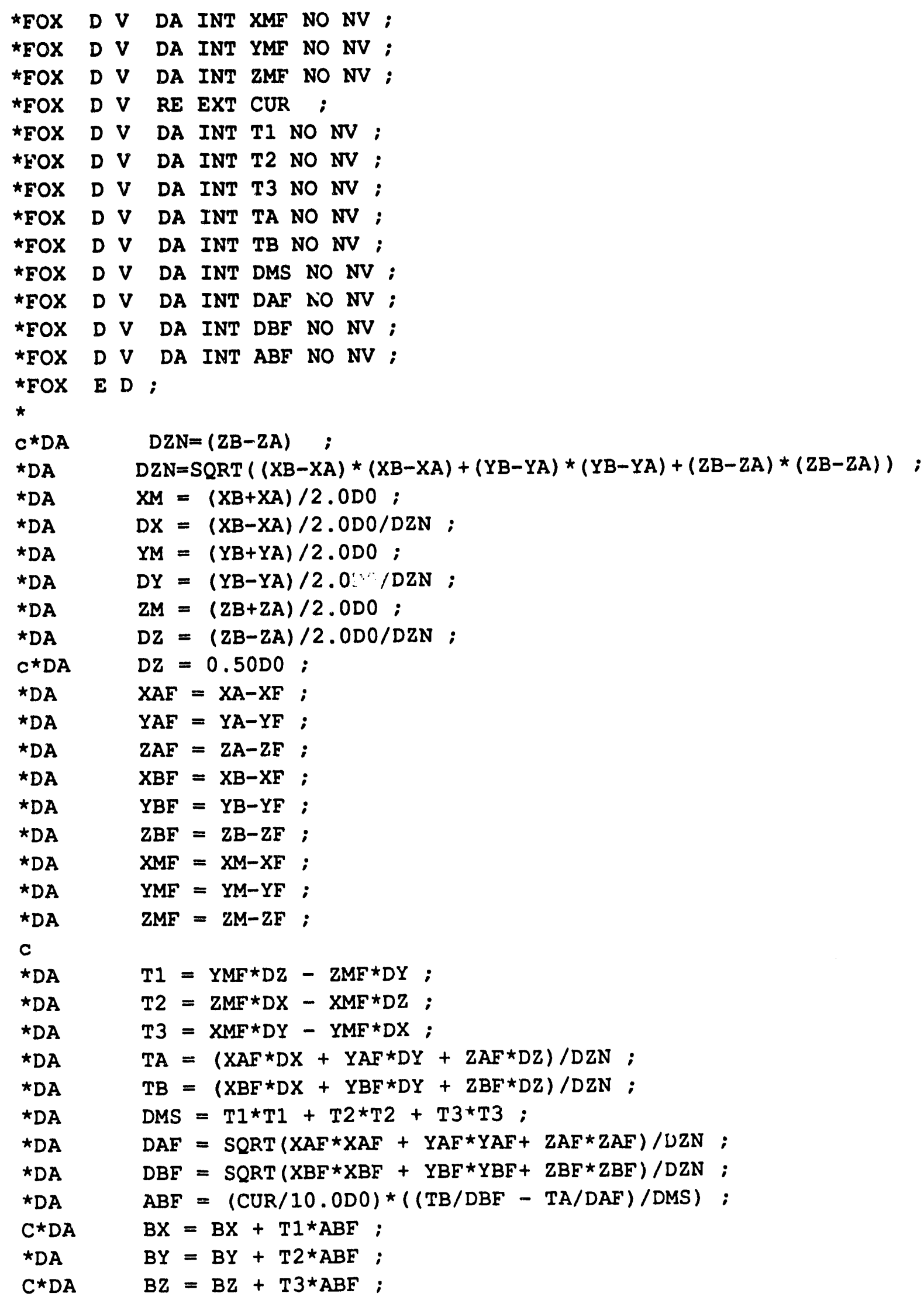




$$
\begin{array}{lr}
c \star D A & Y A=-Y A ; \\
c \star D A & Y B=-Y B ; \\
c & \\
& \text { RETURN }
\end{array}
$$


Acknowledgment

It is a pleasure to acknowledge the profitable discussions we have had with Dr. William

Fawley and Prof. Martin Berz concerning the application of D-A computations, and their resulting magnetic field descriptions, to problems of beam transport. 

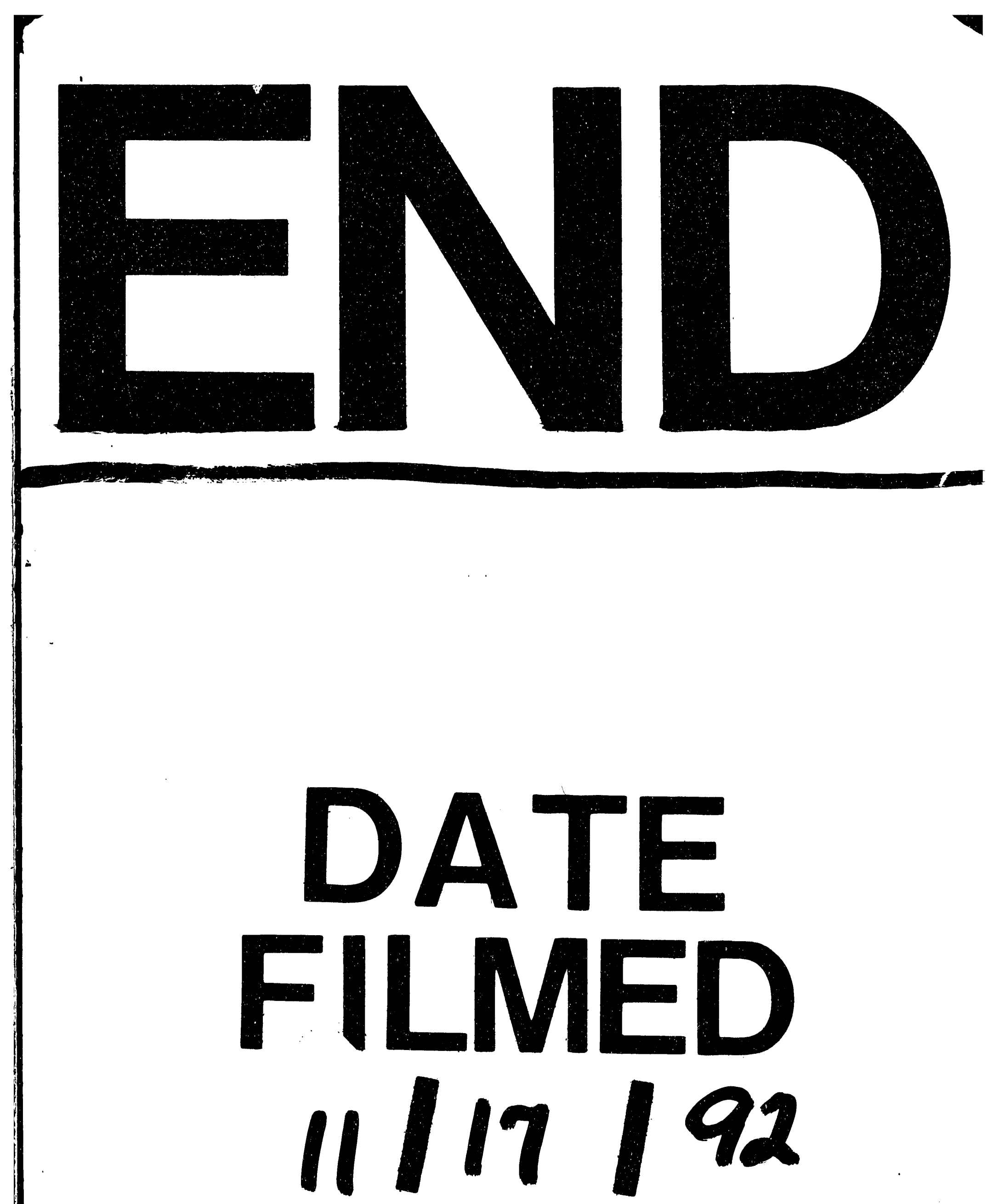
